JOURNAL OF

SYMPLECTIC GEOMETRY

Volume 11, Number 4, 645-701, 2013

\title{
HOMOCLINIC POINTS AND FLOER HOMOLOGY
}

\author{
SONJA HOHLOCH
}

\begin{abstract}
A new relation between homoclinic points and Lagrangian Floer homology is presented: in dimension two, we construct a Floer homology generated by primary homoclinic points. We compute two examples and prove an invariance theorem. Moreover, we establish a link to the (absolute) flux and growth of symplectomorphisms.
\end{abstract}

\section{Introduction}

Homoclinic points are the intersection points of the stable and unstable manifolds of a hyperbolic fixed point. Their discovery goes back to 1889 when Poincaré [Poi1, Poi2] studied the $n$-body problem and came across certain nonconvergent trigonometric series. First results about the nature of homoclinic points are due to Birkhoff [Bi] who discovered an intricate amount of (mostly high) periodic points near homoclinic ones. This phenomenon was formalized by Smale's [Sm1] [Sm2] horseshoe in the 1960s. Melnikov's [Me] perturbation method for producing and detecting homoclinic points also dates to the 1960s. Since the 1970s, genericity properties of homoclinic points were studied by several authors. However, in spite of these achievements, there are still many open questions.

Floer theory is a much more recent development. Floer [F11, F12, Fl3] devised it in the late 1980s when he worked on the Arnold conjecture. Arnold conjectured around 1960 that the number of fixed points of a nondegenerate Hamiltonian diffeomorphism on a closed symplectic manifold is greater or equal to the sum over the Betti numbers. Floer proved Arnold's conjecture first on closed symplectic manifolds with $\pi_{2}(M)=0$ and then on so-called monotone manifolds. After his breakthrough, the conjecture was established on more general closed symplectic manifolds by a series of authors; cf. for references, e.g., Salamon [Sa].

Floer theory is some kind of infinite dimensional Morse theory for the symplectic action functional as a Morse function. It is vividly studied nowadays 
and has many applications not only in symplectic geometry and dynamical systems. The first version of Floer homology was Lagrangian Floer homology. Roughly speaking, its chain groups are generated by the intersection points of two Lagrangian submanifolds. The grading is induced by the Maslov index. The boundary operator counts flow lines of the negative $L^{2}$-gradiant flow of the symplectic action functional between intersection points with Maslov index difference one.

The present paper is motivated by the fact that the stable and unstable manifold of a hyperbolic fixed point of a symplectomorphism are Lagrangian submanifolds. In such a case the homoclinic points can be considered as intersection points of a Lagrangian intersection problem for which one might hope to define a Lagrangian Floer homology. The main obstacle is the wild oscillation and accumulation behaviour of the noncompact Lagrangians. Classical Lagrangian Floer homology is defined for compact Lagrangian submanifolds and can be generalized to "nice" noncompact ones. However, those techniques fail in the present situation.

In order to actually count connecting flow lines one needs compactness of the associated 0-dimensional moduli spaces. If the dimension of the manifold is greater than two this turns out to be a tricky analysis problem about Gromov compactness of spaces of pseudo-holomorphic curves.

However on two-dimensional manifolds, the analysis can be replaced by combinatorics and counting of certain orientation preserving immersions as shown by de Silva $[\mathbf{d S}]$, Fel'shtyn $[\mathbf{F e 1}]$ and Gautschi et al. [GauRS]. Since a symplectic form is a nondegenerate, closed 2-form the notions of "olume preserving" and "symplectic" coincide and symplectomorphisms and volume preserving diffeomorphisms are the same.

The wild behaviour and the noncompactness of the (un)stable manifolds prevent the analysis ansatz. Therefore we will work in a two-dimensional setting. Nevertheless, the set of homoclinic points is still too large to allow a well-defined and meaningful Floer homology as analysed in Hohloch [Ho1]. Our generator set will be the set of so-called primary homoclinic points, which are defined by very rigid geometric properties.

In the following, $(M, \omega)$ stands for $\mathbb{R}^{2}$ or a closed surface with genus $g \geq 1$ with their resp. volume forms. Let $\varphi$ be a symplectomorphism on $M$ with hyperbolic fixed point $x$. Denote the associated stable resp. unstable manifolds by $W^{s}:=W^{s}(x, \varphi)$ resp. $W^{u}:=W^{u}(x, \varphi)$ and set $\mathcal{H}:=W^{s} \cap W^{u}$ to be the set of homoclinic points. Given $p, q \in \mathcal{H}$, we call $[p, q]_{s} \subset W^{s}$ and $[p, q]_{u} \subset W^{u}$ the stable resp. unstable segment between $p$ and $q$. We call $p$ contractible if the loop $[p, x]_{s} \cup[p, x]_{u}$ is contractible in $M$. We denote by $\mathcal{H}_{[x]} \subset \mathcal{H}$ the set of contractible homoclinic points and call

$$
\mathcal{H}_{p r}:=\left\{p \in \mathcal{H}_{[x]} \backslash\{x\} \mid\right] p, x\left[{ }_{s} \cap\right] p, x\left[{ }_{u} \cap \mathcal{H}_{[x]}=\emptyset\right\}
$$


the set of primary points. $\varphi$ induces a $\mathbb{Z}$-action on $\mathcal{H}$ via $\mathbb{Z} \times \mathcal{H} \rightarrow \mathcal{H}$, $(n, p) \mapsto \varphi^{n}(p) . \tilde{\mathcal{H}}_{p r}:=\mathcal{H}_{p r} / \mathbb{Z}$ is finite and we denote the equivalence class or orbit of $p$ by $\langle p\rangle$. The Maslov index $\mu$ induces a grading $\mu: \tilde{\mathcal{H}}_{p r} \rightarrow \mathbb{Z}$. We define $m(p, q)$ to be the number of certain orientation preserving immersions with start point $p$ and end point $q$ and set $m(\langle p\rangle,\langle q\rangle):=\sum_{n \in \mathbb{Z}} m\left(p, \varphi^{n}(q)\right)$.

Theorem 1. The groups and operator

$$
C_{k}(x, \varphi):=\bigoplus_{\substack{\langle p\rangle \in \tilde{\mathcal{H}}_{p r} \\ \mu(\langle p\rangle)=k}} \mathbb{Z}\langle p\rangle \text { and } \partial\langle p\rangle:=\sum_{\substack{\langle q\rangle \in \tilde{\mathcal{H}}_{p r} \\ \mu(\langle q\rangle)=\mu(\langle p\rangle)-1}} m(\langle p\rangle,\langle q\rangle)\langle q\rangle
$$

with $k \in \mathbb{Z}$ form a chain complex, i.e., $\partial \circ \partial=0$, and we call the resulting homology $H_{*}:=H_{*}(x, \varphi):=\frac{\operatorname{ker} \partial_{*}}{\operatorname{Im} \partial_{*+1}}$ primary Floer homology. $C_{k}(x, \varphi)$ and thus $H_{k}(x, \varphi)$ vanish for $k \notin\{ \pm 1, \pm 2, \pm 3\}$.

The well-definedness of $\partial$ and the proof of $\partial \circ \partial=0$ are tricky combinations of dynamical and combinatorial arguments.

$H_{*}$ is invariant under so called contractibly strongly intersecting (symplectic) isotopies (defined later before Theorem 31):

Theorem 2. Let $(M, \omega)$ be a closed symplectic two-dimensional manifold with genus $g \geq 1$. Let $\varphi$ and $\psi$ be symplectomorphisms with hyperbolic fixed points $x \in \operatorname{Fix}(\varphi)$ and $y \in \operatorname{Fix}(\psi)$. Let $(x, \varphi)$ and $(y, \psi)$ be csi and let all primary points of $\varphi$ and $\psi$ be transverse. Assume there is a csi isotopy $\Phi$ from $(x, \varphi)$ to $(y, \psi)$. Then

$$
H_{*}(x, \varphi) \simeq H_{*}(y, \psi) .
$$

The proof has to combine analytical and combinatorial arguments since a primary point $p \in \mathcal{H}_{p r}$ might vanish (analogously arise) in two ways:

(i) $p$ vanishes as intersection point or

(ii) $p$ persists as intersection point, but is no longer primary.

The invariance implies an existence and bifurcation criterion for homoclinic points and the fixed point. Conjecturally Hamiltonian isotopies are naturally strongly intersecting. Moreover, Theorem 2 allows to classify homoclinic tangles up to csi isotopy.

There are several versions of homoclinic Floer homology with quite different flavours. Their well-definedness can be easily deduced from the construction and well-definedness of primary Floer homology.

One version is chaotic Floer homology $\hat{H}_{*}$ which takes into account the periodic points "near" a homoclinic tangle. More precizely, we have a whole sequence $n \mapsto \hat{H}_{*}\left(x, \varphi^{n}\right)$ where $n$ is the number of iterates of the symplectomorphism. For fixed $n$, the boundary operator associated to $\hat{H}_{*}\left(x, \varphi^{n}\right)$ counts only those connecting immersions whose range does not contain any fixed points of $\varphi^{n}$, i.e., $n$-periodic points of $\varphi$. This leads to an interesting 
behaviour of the sequence $n \mapsto \hat{H}_{*}\left(x, \varphi^{n}\right)$ and the definition of a symplectic zeta function

$$
\zeta_{x, \varphi}(z):=\exp \left(\sum_{n=1}^{\infty} \frac{\chi\left(\hat{H}_{*}\left(x, \varphi^{n}\right)\right)}{n} z^{n}\right)
$$

where $\chi\left(\hat{H}_{*}\left(x, \varphi^{n}\right)\right)$ denotes the Euler characteristic of $\hat{H}_{*}\left(x, \varphi^{n}\right)$. The study of this function is an ongoing project.

An important question in symplectic dynamics is the growth behaviour of symplectomorphisms under iteration. Among others, it has been studied extensively by Polterovich [Pol3, Pol4]. By means of the growth behaviour, group theoretic question about the group of Hamiltonian diffeomorphisms can be answered. In [Pol4], a Hamiltonian version of the Zimmer program is devised and the proofs relie on the growth behaviour of iterated Hamiltonian diffeomorphisms.

If we want to study the iteration behaviour of symplectomorphisms by means of primary Floer homology we easily find

$$
\operatorname{rk} H_{*}(x, \varphi) \leq \operatorname{rk} H_{*}\left(x, \varphi^{n}\right) .
$$

For Hamiltonian diffeomorphisms, equality in (3) turns out to be equivalent to proving Theorem 2 for Hamiltonian diffeomorphisms. Thus the strong invariance in Theorem 2 seemingly opposes easy examples with $\operatorname{rk} H_{*}(x, \varphi)<\operatorname{rk} H_{*}\left(x, \varphi^{n}\right)$.

Another version of homoclinic Floer homology, namely semi-primary Floer homology $\tilde{H}_{*}$, is more apt for detecting increasing rank. Instead of primary points, its chain complex is generated by so-called semi-primary points. $\tilde{H}_{*}$ has weaker invariance properties than primary Floer homology. It is easy to find examples with

$$
\tilde{H}_{*}(x, \varphi)<\tilde{H}_{*}\left(x, \varphi^{n}\right) .
$$

Increasing rank of semi-primary Floer homology actually means that parts of the tangle wrap in a certain way around some genus of the surface.

This line of thoughts has been completed in Hohloch [Ho2]. In that work, primary Floer homology on $\mathbb{R}^{2}$ and so-called cylinder Floer homology $\mathscr{H}_{*}$ (a variant of semi-primary Floer homology) on the infinite cylinder $\mathcal{Z}$ are studied using the filtration by the symplectic action $\mathcal{A}$. The action interval of the filtered groups appears as an upper index. The action spectrum is denoted by $\operatorname{Spec}(x, \varphi)$ and the minimal distance between two action levels by $\operatorname{gap}(x, \varphi)$. The boundary operator is modified in such a way that we keep track of the homotopy class on $\mathcal{Z}$.

Theorem 4 ([Ho2]). Let $\varphi \in \operatorname{Symp}\left(\mathbb{R}^{2}\right)$ resp. $\varphi \in \operatorname{Ham}^{c}(\mathcal{Z})$. Let $b \in$ $\operatorname{Spec}(\varphi, x)$ and $0<\varepsilon \leq \frac{1}{2} \operatorname{gap}(\varphi, x)$. Assume that there are $k$ primary classes 
with action b. Then we obtain for the homoclinic Floer homology on $\mathbb{R}^{2}$ resp. $\mathcal{Z}$

$$
\begin{aligned}
& H_{*}^{] b-\varepsilon, b+\varepsilon]}(\varphi, x) \simeq \mathbb{Z}^{k} \quad \text { and } \quad H_{*}^{b b-\varepsilon, b+\varepsilon]}\left(\varphi^{n}, x\right) \simeq\left(\mathbb{Z}^{k}\right)^{n} \\
& \mathscr{H}_{*}{ }^{b-\varepsilon, b+\varepsilon]}(\varphi, x) \simeq \mathbb{Z}^{k} \quad \text { and } \quad \mathscr{H}_{*}^{b-\varepsilon, b+\varepsilon]}\left(\varphi^{n}, x\right) \simeq\left(\mathbb{Z}^{k}\right)^{n} .
\end{aligned}
$$

Thus the rank grows linearly with the number of iterations.

Homoclinic Floer homology is also linked to transport and (absolute) flux of a dynamical system. In the symplectic plane $\left(\mathbb{R}^{2}, \omega\right)$, the absolute flux (briefly flux) of a symplectomorphism $\varphi$ through a simply closed curve $c$ is defined as the volume of the set of points which are swept out of the interior of the curve, i.e.,

$$
\mathcal{F} l u x_{\varphi}(c)=\operatorname{vol}_{\omega}(\varphi(\operatorname{Int}(c)) \cap \operatorname{Ext}(c)) .
$$

It also can be defined for noncontractible curves on the cylinder. Note that this notion is different from the flux homomorphism in symplectic geometry (cf. [McS1,Pol2]) which, roughly speaking, considers the difference between $\varphi(\operatorname{Int}(c)) \cap \operatorname{Ext}(c)$ and $\varphi(\operatorname{Ext}(c)) \cap \operatorname{Int}(c)$.

The absolute flux has been studied by MacKay et al. [MMP] in order to gain a better understanding of the transport. In their setting, transport means the motion of points unter (many) iterations. Invariant curves have zero flux and are therefore complete barriers for the transport. MacKay et al. $[\mathbf{M M P}]$ focus on partially invariant curves associated to cantori, homoclinic and periodic points. In this case, transport is only possible through the noninvariant part of the curve. The non-invariant part forms a so-called turnstile. We generalize this notion in [Ho2] and distinguish between true, overtwisted and generalized turnstiles. Overtwisted turnstiles correspond to mixed moves and generalized turnstiles to primary moves in the prove of Theorem 2.

Proposition 5 ([Ho2]). True and overtwisted turnstiles are annihilated by the boundary operator.

In our setting, the Maslov index and the (relative) action are invariant under iteration of the symplectomorphism. The flux through a homoclinic orbit $\langle p\rangle$ is defined as the flux through a curve parametrizing $[p, x]_{s} \cup[p, x]_{u}$. It transforms

$$
\mathcal{F} l u x_{\varphi^{n}}(\langle p\rangle)=n \mathcal{F} l u x_{\varphi}(\langle p\rangle)
$$

as the Maslov index and action in classical Floer theory, see Ginzburg \& Gürel [GiG]. MacKay et al. [MMP] identified the flux (under certain assumptions) with Mather's [Ma1] difference in action $\triangle W$. If the primary points $p$ and $q$ form a true turnstile and $v \in \mathcal{M}(p, q) \neq \emptyset$ we extend their result to

Theorem 6 ([Ho2]). Under certain assumptions holds

$$
\mathcal{A}(\langle p\rangle)-\mathcal{A}(\langle q\rangle)=\mathcal{A}(\langle p\rangle,\langle q\rangle)=\int_{v} \omega=\mathcal{F} l u x_{\varphi}(\langle p\rangle)=\triangle W_{p, q}
$$


Altogether, $H_{*}$ is the first invariant, which takes the algebraic interaction of homoclinic points into account and links them to dynamical properties like the absolute flux and growth of symplectomorphisms.

\section{Immersions, cutting and gluing}

2.1. Maslov index and homotopy class. In this subsection, we recall the definition of the Maslov index for Lagrangian subspaces in $R^{2 n}$ as it can be found in McDuff and Salamon [McS1]. Using suitable trivialitations, Floer $[$ Fl1 $]$ generalized it to symplectic manifolds. Finally, we introduce crucial notations like (un)stable segments and homotopy classes for homoclinic points.

Denote by $\mathcal{L}(n)$ the space of Lagrangian subspaces of $\left(\mathbb{R}^{2 n}, \omega_{0}\right)$ with $\omega_{0}:=$ $\sum_{i=1}^{n} d x_{i} \wedge d y_{i}$. Represent $\Lambda \in \mathcal{L}(n)$ by $\Lambda=\left(\begin{array}{l}X \\ Y\end{array}\right)$ with $U:=X+\mathrm{i} Y \in$ $U(n)$ and define $\rho: \mathcal{L}(n) \rightarrow S^{1}, \rho(\Lambda):=\operatorname{det}(U \circ U)$. For a loop $\Lambda: \mathbb{R} /$ $\mathbb{Z} \rightarrow \mathcal{L}(n)$, define the Maslov index of loops of Lagrangian subspaces by $\mu(\Lambda):=\operatorname{deg}(\rho \circ \Lambda)$ where deg denotes the mapping degree of $\rho \circ \Lambda: \mathbb{R} /$ $\mathbb{Z} \rightarrow S^{1}$. If $\alpha: \mathbb{R} \rightarrow \mathbb{R}$ is a lift of $\rho \circ \Lambda$, i.e., $\operatorname{det}(X(t)+i Y(t))=\mathrm{e}^{\mathrm{i} \pi \alpha(t)}$, we obtain $\mu(\Lambda)=\alpha(1)-\alpha(0)$.

Let $(M, \omega)$ be a $2 n$-dimensional symplectic manifold and $\varphi$ a symplectomorphism with hyperbolic fixed point $x$. For symplectomorphisms, the (un)stable manifolds $W^{u}:=W^{u}(x, \varphi)$ and $W^{s}:=W^{s}(x, \varphi)$ are Lagrangian submanifolds and there are injective immersions $\gamma_{u}: \mathbb{R}^{n} \rightarrow W^{u}$ and $\gamma_{s}: \mathbb{R}^{n} \rightarrow W^{s}$ with $\gamma_{u}(0)=x=\gamma_{s}(0)$. Provide $\mathcal{P}\left(W^{u}, W^{s}\right):=\{\beta:[0,1] \rightarrow$ $\left.M \mid \beta(0) \in W^{u}, \beta(1) \in W^{s}\right\}$ with the smallest topology such that the following three maps are continuous:

$$
\begin{array}{lll}
\mathcal{P}\left(W^{u}, W^{s}\right) \rightarrow C([0,1] ; M), & \beta \mapsto \beta, \\
\mathcal{P}\left(W^{u}, W^{s}\right) \rightarrow \mathbb{R}, & \beta \mapsto \gamma_{u}^{-1}(\beta(0)), \\
\mathcal{P}\left(W^{u}, W^{s}\right) \rightarrow \mathbb{R}, & \beta \mapsto \gamma_{s}^{-1}(\beta(1)) .
\end{array}
$$

Fix $\alpha \in \mathcal{P}\left(W^{u}, W^{s}\right)$ and denote its connected component by $\mathcal{P}_{\alpha}\left(W^{u}, W^{s}\right)$. Identify $p, q \in \mathcal{H}$ as constant paths in $\mathcal{P}_{\alpha}\left(W^{u}, W^{s}\right)$. Let $v:[0,1] \rightarrow$ $\mathcal{P}\left(W^{u}, W^{s}\right)$ with $v(0) \equiv p$ and $v(1) \equiv q$ and see it as map $v:[0,1]^{2} \rightarrow M$ via $v(s, t):=v(s)(t)$. The square $[0,1]^{2}$ is contractible and we can find a trivialization $\Phi:=\Phi_{v}: v^{*} T M \rightarrow[0,1]^{2} \times \mathbb{R}^{2 n}$ such that the symplectic form on the fibres is mapped to the standard $\omega_{0}$ on $\mathbb{R}^{2 n} \simeq \mathbb{C}^{n}, \Phi$ is constant on $\{0\} \times[0,1]$ and on $\{1\} \times[0,1]$, and $\Phi\left(T_{p} W^{s}\right)=\mathrm{i} \Phi\left(T_{p} W^{u}\right)$ and $\Phi\left(T_{q} W^{s}\right)=\mathrm{i} \Phi\left(T_{q} W^{u}\right)$.

Denote by $\partial[0,1]^{2}$ the boundary of $[0,1]^{2}$ and define the loop $\Lambda_{v}$ : $\partial[0,1]^{2} \rightarrow \mathcal{L}(n)$ starting in $(0,0)$ and running through $(1,0),(1,1)$ and 
$(0,1)$ back to $(0,0)$ piecewise via

$$
\begin{array}{ll}
(\xi, 0) \mapsto \Phi\left(T_{v(\xi, 0)} W^{u}\right), & (\xi, 1) \mapsto \Phi\left(T_{v(\xi, 1)} W^{s}\right), \\
(1, \eta) \mapsto \mathrm{e}^{\frac{i \pi \eta}{2}} \Phi\left(T_{q} W^{u}\right), & (0, \eta) \mapsto \mathrm{e}^{\frac{i \pi(\eta-1)}{2}} \Phi\left(T_{p} W^{s}\right) .
\end{array}
$$

Under the above conventions, we define the relative Maslov index for $p$, $q \in \mathcal{H}$ via $\mu(p, q):=\mu\left(\Lambda_{v}\right)$. If $\pi_{2}(M)=0$, then $\left.c_{1}\right|_{\pi_{2}(M)}=0$ (where $c_{1}$ denotes the first Chern class of $M$ ) and the construction is independent from the chosen path $v$ and the trivialization $\Phi$. Concerning the two-dimensional situation, recall that the second homotopy class of a closed surfaces with genus $g \geq 1$ always vanishes.

From now on, $(M, \omega)$ is either $\left(\mathbb{R}^{2}, d x \wedge d y\right)$ or a closed, two-dimensional manifold with genus $g \geq 1$. For $i \in\{u, s\}$ the immersions $\gamma_{i}: \mathbb{R} \rightarrow W^{i}$ induce an ordering $<_{i}$ resp. $\leq_{i}$ on $W^{i}$ via

$$
\gamma_{i}(t)<_{i} \gamma_{i}(\tilde{t}) \Leftrightarrow t<\tilde{t} \quad \operatorname{resp.} \quad \gamma_{i}(t) \leq_{i} \gamma_{i}(\tilde{t}) \Leftrightarrow t \leq \tilde{t} .
$$

By abuse of notation, we say that $p, q \in W^{i}$ induce an ordering on $W^{i}$ via setting $p<_{i} q$ resp. $p \leq_{i} q$. For $i \in\{0,1\}$ consider $p, q \in W^{i}$ and set $t_{i}^{p}=\gamma_{i}^{-1}(p), t_{i}^{q}:=\gamma_{i}^{-1}(q), t_{i}^{-}:=\min \left\{t_{i}^{p}, t_{i}^{q}\right\}$ and $t_{i}^{+}:=\max \left\{t_{i}^{p}, t_{i}^{q}\right\}$. We call

$$
[p, q]_{u}:=\gamma_{u}\left(\left[t_{u}^{-}, t_{u}^{+}\right]\right) \quad \text { resp. } \quad[p, q]_{s}:=\gamma_{s}\left(\left[t_{s}^{-}, t_{s}^{+}\right]\right)
$$

the segments in $W^{u}$ resp. $W^{s}$ between $p$ and $q$. The segments are independent of the chosen immersion and a priori just sets of points, thus $[p, q]_{i}=[q, p]_{i}$. Analogously, we define the open and half-open segments $] p, q[i$ and $[p, q[i$.

Now we assign to each $p \in \mathcal{H}$ a homotopy class in $\pi_{0}\left(\mathcal{P}\left(W^{u}, W^{s}\right)\right) \simeq$ $\pi_{1}(M, x)$ : Denote by $c_{p}:[0,1] \rightarrow W^{u} \cup W^{s}$ a curve with $c_{p}(0)=x=c_{p}(1)$ which runs through $[x, p]_{u}$ to $p$ and through $[p, x]_{s}$ back to $x$. Set $[p]:=$ $\left[c_{p}\right] \in \pi_{1}(M, x)$ and $[-p]$ for the path with the inverse parametrization. Then $\mathcal{H}_{[x]}:=\{p \in \mathcal{H} \mid[p]=[x]\}$ is the set of contractible homoclinic points. $\mathcal{H}_{[x]}$ is invariant under the action of $\varphi$. Moreover, if $\varphi=\varphi_{1}$ is the time-1 map of a flow and $\xi: S^{1} \rightarrow M, \xi(t):=\varphi_{t}(x)$ and $\xi$ is contractible or $\pi_{1}(M, x)$ abelian then $[p]=\left[\varphi_{1}^{n}(p)\right]$ for all $p \in \mathcal{H}$ and $n \in \mathbb{Z}$.

Remark 7. For contractible $p, \tilde{p}, q \in \mathcal{H}$, we observe:

(1) $\mu(q, p)=-\mu(p, q)$ and $\mu(p, q)+\mu(q, \tilde{p})=\mu(p, \tilde{p})$.

(2) $\mu(p, q)=\mu\left(\varphi^{n}(p), \varphi^{n}(q)\right)$ for $n \in \mathbb{Z}$, i.e., the (relative) Maslov index of $p$ and $q$ is invariant under the $\mathbb{Z}$-action of $\varphi$ on $\mathcal{H}$.

(3) $\mu\left(p, \varphi^{n}(p)\right)=0$ for all $n \in \mathbb{Z}$.

(4) $\mu(p, q)=\mu\left(p, \varphi^{n}(q)\right)$ for $n \in \mathbb{Z}$.

The (relative) Maslov index yields a grading $\mu: \mathcal{H}_{[x]} \rightarrow \mathbb{Z}$ via $\mu(p):=$ $\mu(p, x)$ such that for contractible $p$ and $q$ holds $\mu(p, q)=\mu(p, x)+\mu(x, q)=$ $\mu(p, x)-\mu(q, x)=\mu(p)-\mu(q)$. 

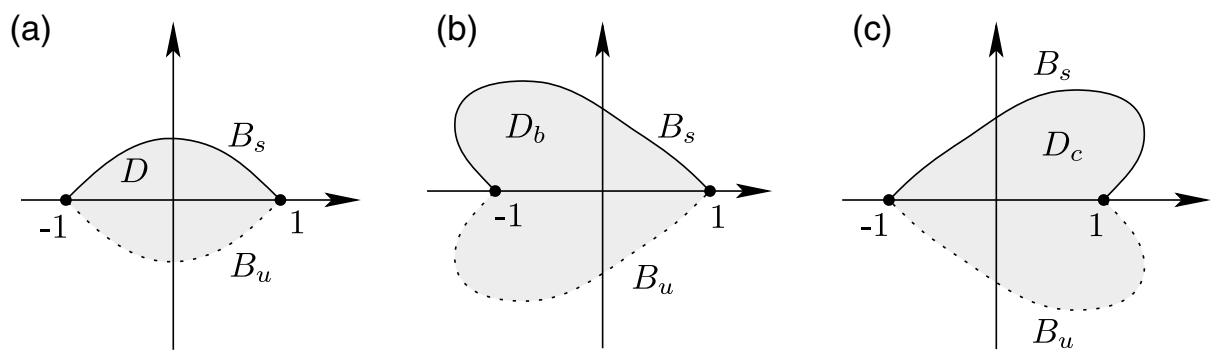

Figure 1. Di-gon and heart.

Remark 8. Let $\tau:(\tilde{M}, \tilde{\omega}) \rightarrow(M, \omega)$ be the universal cover with $\tau^{*} \omega=\tilde{\omega}$ and $p, q \in \mathcal{H}$ with $[p]=[q]$. Denote by $[\tilde{p}, \tilde{q}]_{i}$ the lift of $[p, q]_{i}$ to the universal cover $(\tilde{M}, \tilde{\omega})$ starting in $\tilde{p} \in \tau^{-1}(p)$. Then $\mu(p, q)=\mu(\tilde{p}, \tilde{q})$.

2.2. Immersions, di-gons and hearts. This subsection introduces certain di-gons, also known as 2-gons, lunes or half-moons (Chekanov [Che], de Silva $[\mathbf{d S}]$, Gautschi et al. [GauRS], Robbin [Ro]). They will be crucial for the definition of the boundary operator of the Floer chain complex.

A di-gon is the polygon $D \subset \mathbb{R}^{2}$ with two convex vertices at $(-1,0)$ and $(1,0)$ sketched in Figure 1 (a). Denote its upper boundary by $B_{s}$ and its lower boundary by $B_{u}$.

A heart is either the polygon $D_{b}$ of Figure 1 (b) or the polygon $D_{c}$ of Figure $1(\mathrm{c})$. A heart is characterized by two vertices at $(-1,0)$ and $(1,0)$ where one is convex and one concave. Denote their upper boundaries by $B_{s}$ and their lower boundaries by $B_{u}$.

We require the immersions in the following definitions to be immersions also on the boundaries and vertices. Thus the image of a small neighbourhood of a convex (concave) vertex of a polygon is a wedge-shaped region with angle smaller (larger) than $\pi$.

Definition 9. Let $D$ be the digon and $p, q \in \mathcal{H}$ with $\mu(p, q)=1$. We define $\mathcal{M}(p, q)$ to be the space of smooth, immersed di-gons $v: D \rightarrow M$, which are orientation preserving and satisfy $v\left(B_{u}\right) \subset W^{u}, v\left(B_{s}\right) \subset W^{s}, v((-1,0))=p$ and $v((1,0))=q$. Denote by $G(D)$ the group of orientation preserving diffeomorphisms of $D$, which preserve the vertices and call $\widehat{\mathcal{M}}(p, q):=\mathcal{M}(p, q) /$ $G(D)$ the space of unparametrized immersed di-gons.

Since there is exactly one segment $[p, q]_{i}, i \in\{s, u\}$, joining $p, q \in \mathcal{H}$ and since $\pi_{2}(M)=0$ we deduce $\# \widehat{\mathcal{M}}(p, q) \in\{0,1\}$ for $p$ and $q$ with $\mu(p, q)=1$.

Definition 10. Consider the hearts $D_{b}$ and $D_{c}$ and $p, q \in \mathcal{H}$ with $\mu(p, q)=$ 2. We define $\mathcal{N}_{b}(p, q)$ resp. $\mathcal{N}_{c}(p, q)$ to be the space of smooth immersed hearts $w: D_{b} \rightarrow M$ resp. $w: D_{c} \rightarrow M$ which are orientation preserving and satisfy $w\left(B_{u}\right) \subset W^{u}, w\left(B_{s}\right) \subset W^{s}, w(-1,0)=p$ and $w(1,0)=q$. We set 
$\mathcal{N}(p, q):=\mathcal{N}_{b}(p, q) \cup \dot{\mathcal{N}}(p, q)$. Denote by $G\left(D_{b}\right)$ resp. $G\left(D_{c}\right)$ the group of orientation preserving diffeomorphisms of $D_{b}$ resp. $D_{c}$, which preserve the vertices and let $\widehat{\mathcal{N}}_{b}(p, q):=\mathcal{N}_{b}(p, q) / G\left(D_{b}\right)$ resp. $\widehat{\mathcal{N}}_{c}(p, q):=\mathcal{N}_{c}(p, q) /$ $G\left(D_{c}\right)$ and $\widehat{\mathcal{N}}(p, q):=\widehat{\mathcal{N}}_{b}(p, q) \dot{\cup} \widehat{\mathcal{N}}_{c}(p, q)$ be the spaces of unparametrized immersed hearts.

If we work with the spaces $\mathcal{M}(p, q)$ and $\mathcal{N}(p, r)$ we always implicitly assume $p, q, r \in \mathcal{H}$ with $[p]=[q],[p]=[r], \mu(p, q)=1$ and $\mu(p, r)=2$.

2.3. Winding number. In the following, we define the winding number for digons and hearts. It will be used for analysing and classification purposes.

Consider the universal cover $\tau: \tilde{M} \rightarrow M$ with induced orientation as topological manifold. For all $\tilde{z} \in \tilde{M}$, the orientation induces an isomorphism $H_{2}(\tilde{M}, \tilde{M} \backslash\{\tilde{z}\}) \simeq \mathbb{Z}$ and the contractibility of $\tilde{M} \simeq \mathbb{R}^{2}$ implies $H_{1}(\tilde{M} \backslash\{\tilde{z}\}) \simeq H_{2}(\tilde{M}, \tilde{M} \backslash\{\tilde{z}\})$. Denote the fundamental class of $S^{1}$ by $\left[S^{1}\right]$. Now consider a continuous path $\tilde{\gamma}: S^{1} \rightarrow \tilde{M}$ and $\tilde{z} \in \tilde{M} \backslash \operatorname{Im}(\tilde{\gamma})$. We define the winding number of $\tilde{\gamma}$ w.r.t. $\tilde{z}$ by $\operatorname{Ind}_{\tilde{\gamma}}(\tilde{z}):=\tilde{\gamma}_{*}\left(\left[S^{1}\right]\right) \in H_{1}(M \backslash\{\tilde{z}\}) \simeq \mathbb{Z}$.

Identifying $\tilde{M}$ with $\mathbb{R}^{2}$ by an orientation preserving diffeomorphism, the winding number also can be seen as mapping degree of $S^{1} \rightarrow S^{1}, t \mapsto \frac{\tilde{\gamma}(t)-\tilde{z}}{|\tilde{\gamma}(t)-\tilde{z}|}$.

Definition 11. Let $A$ stand for $D, D_{b}$ or $D_{c}$ and consider $v: A \rightarrow M$ and a lift $\tilde{v}: A \rightarrow \tilde{M}$ of $v$. The winding number $\operatorname{Ind}_{\tilde{v}}(\tilde{z})$ of $\tilde{v}$ w.r.t. $\tilde{z} \in$ $\tilde{M} \backslash \tilde{v}(\partial A)$ is defined as the winding number of the path $\left.\tilde{v}\right|_{\partial A}$ w.r.t. $\tilde{z}$ with $\partial A$ parametrized counterclockwise.

The winding number of $v$ w.r.t. $z \in M \backslash v(\partial A)$ is defined as

$$
\operatorname{Ind}_{v}(z):=\sum_{\tilde{z} \in \tau^{-1}(z)} \operatorname{Ind}_{\tilde{v}}(\tilde{z})
$$

since $\operatorname{Ind}_{\tilde{v}}$ vanishes for all $\tilde{z}$ lying in the unbounded component of $\tilde{M} \backslash \tilde{v}(\partial A)$.

There is another way to compute the winding number of $\tilde{v}: A \rightarrow \tilde{M}$ : For $\tilde{z} \in \tilde{M} \backslash \tilde{v}(\partial A)$ let $\tilde{B}$ be a small ball around $\tilde{z}$ and similarly consider small balls $B_{i}$ around the $z_{i} \in \tau^{-1}(\tilde{z})$. Identify $\partial \tilde{B} \simeq S^{1} \simeq \partial B_{i}$ and set $\hat{A}:=A \backslash\left(\bigcup_{z_{i} \in \tau^{-1}(\tilde{z})} B_{i}\right)$. Then using some kind of 'local degree' (see Bredon $[\mathbf{B r}])$ we obtain

$$
\operatorname{Ind}_{\tilde{v}}(\tilde{z})=\operatorname{deg}(\partial A \rightarrow \partial \tilde{B})=\operatorname{deg}(\partial \hat{A} \rightarrow \partial \tilde{B})+\operatorname{deg}\left(\bigcup_{z_{i} \in \tau^{-1}(\tilde{z})} \partial B_{i} \rightarrow \partial \tilde{B}\right)
$$

Now if $N$ and $P$ are compact orientable manifolds of dimension $n$ without boundary and if a smooth $\alpha: N \rightarrow P$ can be extended smoothly to some $(n+1)$-dimensional manifold $Q$ with $\partial Q=N$ then $\operatorname{deg}(\alpha)=0$ (see for example Milnor [Mi] ). Recognizing $\hat{A}$ as $Q$ and $\left(\bigcup_{z_{i} \in \tau^{-1}(\tilde{z})} \partial B_{i}\right) \cup \partial A$ as $N$ 
we deduce $\operatorname{deg}(\partial \hat{A} \rightarrow \partial \tilde{B})=0$ whereas the term $\operatorname{deg}\left(\bigcup_{z_{i} \in \tau^{-1}(\tilde{z})} \partial B_{i} \rightarrow \partial \tilde{B}\right)$ yields for orientation preserving immersions:

Remark 12. For $v \in \mathcal{M}(p, q)$ and $z \in M \backslash v(\partial D)$ holds $\operatorname{Ind}_{v}(z)=\# v^{-1}(z)$ and therefore in particular $\operatorname{Ind}_{v} \geq 0$. The analogous result is true for $v \in$ $\mathcal{N}(p, r)$. If there is a component of $M \backslash v(\partial A)$ with $\operatorname{Ind}_{v}<0$ then $v$ is no immersion.

The union of those components of $M \backslash v(\partial D)$ with $\operatorname{Ind}_{v}>0$ is called the interior $\operatorname{Int}(v)$ of $v$. The union of the others is called the exterior $\operatorname{Ext}(v)$ of $v$ (their winding number vanishes).

The following remark will be needed for the existence of the "cutting points" in the cutting construction Theorem 15 . There we will need to know that the vertices of an immersion are not multiply covered. Now choose a metric on $M$. Since the image of our immersions stays in a compact region the following does not depend on the choice of the metric.

Remark 13. Let $v \in \mathcal{M}(p, q)$. Then there is $\varepsilon>0$ such that $U_{p}:=$ $v^{-1}\left(B_{\varepsilon}(p)\right)$ is a connected neighbourhood of $(-1,0) \in D$ with $\left.v\right|_{U_{p}}$ injective. As a consequence $\operatorname{Ind}_{v}=1$ on $B_{\varepsilon}(p) \cap v(D)$ and $v\left(U_{p}\right)$ is the wedge-shaped piece of $B_{\varepsilon}(p)$ bounded by $\left([p, q]_{u} \cup[p, q]_{s}\right) \cap B_{\varepsilon}(p)$ with angle $<\pi$. exterior of $v$. An analogous statement is true for $q$. For $v \in \mathcal{N}(p, r)$ with vertices $p$ and $r$ the only change is $>\pi$ for the concave vertex.

Here the lack of self-intersections of $W^{u}$ and $W^{s}$ plays an important role - otherwise the statement is not true.

2.4. Gluing and cutting. Briefly, gluing of two immersed di-gons $v \in$ $\widehat{\mathcal{M}}(p, q)$ and $\hat{v} \in \widehat{\mathcal{M}}(q, r)$ with $\mu(p, q)=1=\mu(q, r)$ (and therefore $\mu(p, r)=$ $2)$ is the construction which recognizes the tupel $(v, \hat{v})$ as an element of $\widehat{\mathcal{N}}(p, r)$. Cutting is the 'inverse' construction which starts with $w \in \widehat{\mathcal{N}}(p, r)$ and finds two significant points $q_{u}, q_{s} \in \mathcal{H}$ such that $w$ can be seen either as tupel $(v, \hat{v}) \in \widehat{\mathcal{M}}\left(p, q_{u}\right) \times \widehat{\mathcal{M}}\left(q_{u}, r\right)$ or as tupel $\left(v^{\prime}, \hat{v}^{\prime}\right) \in \widehat{\mathcal{M}}\left(p, q_{s}\right) \times \widehat{\mathcal{M}}\left(q_{s}, r\right)$.

Theorem 14 (Gluing). Let $p, q, r \in \mathcal{H}$ with $[p]=[q]=[r]$ and $\mu(p, q)=$ $1=\mu(q, r)$. Let $v \in \widehat{\mathcal{M}}(p, q)$ and $\hat{v} \in \widehat{\mathcal{M}}(q, r)$. Then the gluing procedure \# for $v$ and $\hat{v}$ yields an immersed heart $w:=\hat{v} \# v \in \widehat{\mathcal{N}}(p, r)$.

Proof. The four possible geometric positions of the three involved points are described in Figure 2. The $q$ which lies on that part of the unstable manifold, which crossed the interior of the immersed heart after passing the concave vertex, is called $q_{u}$ and analogously for $q_{s}$. The gluing construction \# glues $v \in \mathcal{M}\left(p, q_{u}\right)$ and $\hat{v} \in \mathcal{M}\left(q_{u}, r\right)$ along the common boundary segment $\left[p, q_{u}\right]_{u}$. For technical details see Chekanov [Che]. 

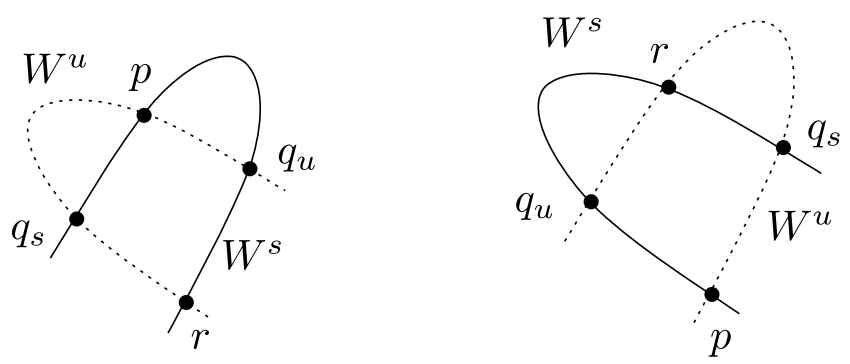

Figure 2. Immersions with $\mu(p)=\mu(q)+1=\mu(r)+2$.

We call the two connected components of $W^{s} \backslash\{x\}$ resp. $W^{u} \backslash\{x\}$ the branches of the (un)stable manifolds. $W^{u}$ and $W^{s}$ are called strongly intersecting (w.r.t. $x$ ) if each branch of $W^{u}$ intersects each branch of $W^{s}$, i.e., $W_{+}^{i} \cap W_{j}^{+} \neq \emptyset \neq W_{-}^{i} \cap W_{j}^{+}$for $i, j \in\{0,1\}$ and $i \neq j$.

To be strongly intersecting is generic in $C^{1}$-topology on closed $n$ dimensional manifolds (Takens $[\mathbf{T a}]$, Xia [Xia1]). For $C^{r}$-topology with $1 \leq r \leq \infty$ there are results by Robinson, Pixton and Oliveira on $S^{2}$ and $T^{2}$. If the action of the symplectomorphism on the first homology group is irreducible then Oliveira $[\mathbf{O l}]$ proved $C^{r}$-genericity for closed surfaces with genus $g \geq 2$. This hypothesis is not fulfilled by symplectomorphisms isotopic to the identity (for example Hamiltonian diffeomorphisms). For the latter ones, Xia [Xia3] proved strongly intersecting to be $C^{r}$-generic on closed surfaces.

Theorem 15 (Cutting). Let $W^{u}$ and $W^{s}$ be strongly intersecting and transverse. Let $p, r \in \mathcal{H}$ with $[p]=[r]$ and $\mu(p, r)=2$ and $w \in \mathcal{N}(p, r)$. Then there are distinct, unique $q_{u}, q_{s} \in \mathcal{H}$ with $\mu\left(p, q_{i}\right)=1=\mu\left(q_{i}, r\right)$ and $v_{i} \in \mathcal{M}\left(p, q_{i}\right), \hat{v}_{i} \in \mathcal{M}\left(q_{i}, r\right)$ such that $\hat{v}_{i} \# v_{i}=w$ for $i \in\{s, u\}$.

Note that a symplectomorphism $\varphi$ is either orientation preserving on the stable and unstable manifold or orientation reversing on both. In the first case we call $\varphi W$-orientation preserving and in the latter one $W$-orientation reversing.

Moreover recall Palis' $\lambda$-lemma [Pa]: Given a small $\operatorname{dim} W^{u}$-dimensional $\operatorname{disc} D^{u} \subset W^{u}(x, \varphi)$ centred around $x, p \in W^{s}(x, \varphi)$ and a $\operatorname{dim} W^{u_{-}}$ dimensional disc $D$ around $p$ intersecting $W^{s}(x, \varphi)$ transversely, then $\bigcup_{n \geq 0} \varphi^{n}(D)$ contains an $m_{u}$-disc arbitrarily $C^{k}$-close to $D^{u}$.

The proof of the cutting construction differs considerably from the one for compact Lagrangian submanifolds which can be found in Chekanov [Che], de Silva [dS], Gautschi et al. [GauRS] or Robbin [Ro]. 
(a)

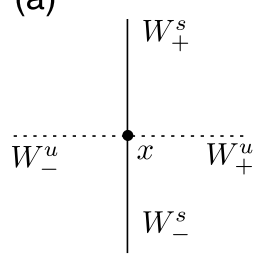

Our convention (b)

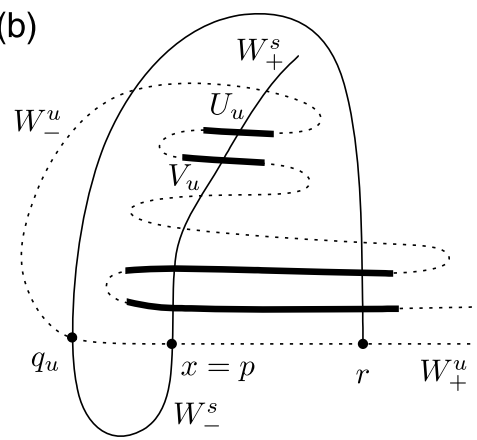

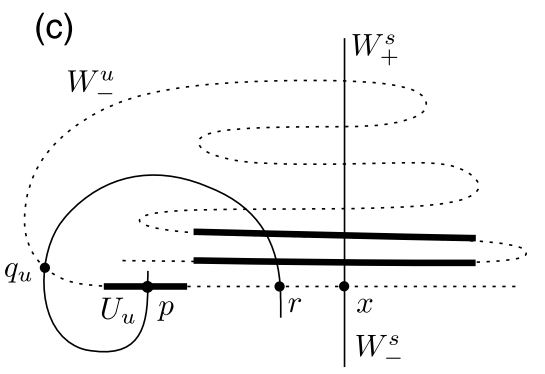

(d)

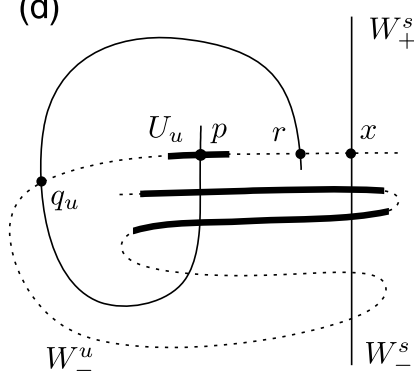

Figure 3. Constructions for $q_{u}$.

Proof. Let us start with the $W$-orientation preserving case and assume $p$ to be the concave vertex of $w \in \mathcal{N}(p, r)$, thus $w: D_{b} \rightarrow M$. Let w.l.o.g. $r<_{i} p$ for $i \in\{s, u\}$.

Given a small disc neighbourhood $D(x)$ of $x$ in $W^{u}$ there is $n \in \mathbb{N}$ such that $\varphi^{-n}(p)$ and $\varphi^{-n}(r)$ lie in $D(x)$. If we can prove the existence of 'cutting points' $q_{u}$ and $q_{s}$ for $\varphi^{-n}(p), \varphi^{-n}(r)$ and $\varphi^{-n} \circ w$ then $\varphi^{n}\left(q_{u}\right)$ and $\varphi^{n}\left(q_{s}\right)$ are cutting points for $p, r$ and $w$.

Now choose $D(x)$ to be the 'convergence disc' $D^{u} \subset W^{u}$ of the $\lambda$-lemma and assume from now on w.l.o.g. $p, r \in D^{u}$.

In order to find the cutting point $q_{u}$, we follow the segment $\left[p, \infty\left[{ }_{u}\right.\right.$. For a certain time after $p$, it stays in the interior of $w$. We will prove that at some point it passes $w\left(\partial D_{b}\right)$ to the exterior of $w$ and that the first such point will be our desired $q_{u}$. We define

$$
q_{u}:=\min \left\{q \in W^{u} \mid p<_{u} q, q \in\right] r, p\left[s,\left[q, q+\varepsilon\left[{ }_{u} \cap w\left(D_{b}\right)^{c} \neq \emptyset \text { for } \varepsilon>0\right\}\right.\right.
$$

where the last condition deals with the possible lack of global injectivity. Now we prove that such a minimum always exists. We use the notation for the branches $W_{ \pm}^{u}$ and $W_{ \pm}^{s}$ as sketched in Figure 3 (a).

Case $p=x \neq r$ : For the relative positions of $p$ and $r$ see Figure $3(\mathrm{~b}) . W_{+}^{u}$ is the branch of $W^{u}$ containing $r$. $W_{+}^{s}$ is the branch of $W^{s}$ which starts in the 
local picture on the same side of $W^{u}$ as $[r, r+\varepsilon]_{s}$ for small $\varepsilon>0$. Since $W^{u}$ and $W^{s}$ are strongly intersecting and transverse there is $q \in W_{-}^{u} \pitchfork W_{+}^{s}$ with $p<_{u} q$. Let $t_{i}^{q}:=\gamma_{i}^{-1}(q)$ and consider a small neighbourhood of $q$ in $W_{-}^{u}$. If $\operatorname{sign}\left(\operatorname{det}\left(\dot{\gamma}_{u}\left(t_{u}^{q}\right), \dot{\gamma}_{s}\left(t_{s}^{q}\right)\right)\right)$ is negative we denote the neighbourhood by $U_{u}$ and otherwise by $V_{u}$. These neighbourhoods meet $W^{s}$ transversely in $q$ such that the $\lambda$-lemma implies the $C^{k}$-convergence of discs $D_{n} \subset \varphi^{n}\left(U_{u}\right)$ resp. $D_{n} \subset \varphi^{n}\left(V_{u}\right)$ to $D^{u}$ for $n \rightarrow \infty$. Recall that $r \in D^{u}$ and that $[r, p]_{s}$ intersects $W^{u}$ in $r$ transversely. Thus for given $\varepsilon>0$, there is $n_{0}$ large enough such that $D_{n}$ and $[r, r+\varepsilon]_{s}$ intersect for $n \geq n_{0}$, see the extra bold long segments in Figure 3 (b). Remark 13 states that, for $\varepsilon>0$ small enough, the ball $B_{\varepsilon}(r)$ splits into two wedge-shaped regions $W_{\text {int }} \subset \operatorname{Int}(w)$ and $W_{\text {ext }} \subset \operatorname{Ext}(w)$ with common boundary $\left([r, p]_{u} \cup[r, p]_{s}\right) \cap B_{\varepsilon}(r)$.

Thus for $n$ large enough, $D_{n}$ meets $W_{\text {ext }}$ before or after passing $] r, r+\varepsilon[s$ depending on if $D_{n}$ lies in an iterate of $U_{u}$ or $V_{u}$. Therefore, the segment $\left[p, \infty\left[{ }_{u}\right.\right.$ leaves $\operatorname{Int}(w)$ and meets $\operatorname{Ext}(w)$ such that points as claimed in the definition of $q_{u}$ exist and so does the minimum $q_{u}$.

Case $p \neq x$. Here we do not need the (un)stable manifolds to be strongly intersecting. The sketches in Figure 3 are schematical and it is unimportant if $x$ lies in the exterior of $w$ or not. There are two subcases, namely if $p \in$ $W_{-}^{u} \pitchfork W_{+}^{s}$ as in (c) or if $p \in W_{-}^{u} \pitchfork W_{-}^{s}$ as in (d).

We start with $p \in W_{-}^{u} \pitchfork W_{+}^{s}$ and consider a small neighbourhood around $p$ in $W_{-}^{u}$. If $\operatorname{sign}\left(\operatorname{det}\left(\dot{\gamma}_{u}\left(t_{u}^{p}\right), \dot{\gamma}_{s}\left(t_{s}^{p}\right)\right)\right)$ is negative we denote the small neighbourhood by $U_{u}$ and otherwise by $V_{u}$. Since $p \in W_{+}^{s}$, the discs $D_{n} \subset \varphi^{n}\left(U_{u}\right)$ resp. $D_{n} \subset \varphi^{n}\left(V_{u}\right)$ approach the "convergence disc" $D^{u}$ centred at $x$ from the $W_{+}^{s}$-side for $n \rightarrow \infty$, see the extra bold long segments in Figure 3 (c). As in the proof of case $p=x$, we consider the special neighbourhood sectors $W_{\text {int }} \subset \operatorname{Int}(w)$ and $W_{\text {ext }} \subset \operatorname{Ext}(w)$ of $r$ provided by Remark 13 . We conclude that $] p, \infty\left[{ }_{u}\right.$ passes somewhere through $W_{\text {ext }}$ and therefore meets the exterior of $w$. Thus points as claimed in the definition of $q_{u}$ exist and so does the minimum $q_{u}$.

Case $p \in W_{-}^{u} \pitchfork W_{-}^{s}$ (sketched in Figure $3(\mathrm{~d})$ ) proceeds analogously to case $p \in W_{-}^{u} \pitchfork W_{+}^{s}$ except from the following fact: Now the discs $D_{n} \subset$ $\varphi^{n}\left(U_{u}\right)$ resp. $D_{n} \subset \varphi^{n}\left(V_{u}\right)$ approach the 'convergence disc' $D^{u}$ from the $W_{-}^{s}$-side for $n \rightarrow \infty$, see the extra bold long segments in Figure 3 (d). Therefore we have to use the sectors $W_{\text {int }} \subset \operatorname{Int}(w)$ and $W_{\text {ext }} \subset \operatorname{Ext}(w)$ of $p$ instead of those of $r$ and then proceed as above.

Since we only need the oscillation behaviour predicted by the $\lambda$-lemma and those special neighbourhood sectors around the vertices the proof carries over to all possible relative positions of $x, p$ and $r$ within $W^{u}$ and $W^{s}$ in case $p \neq x$.

Exchanging the roles of $W^{u}$ and $W^{s}$, the constructions for $q_{s}$ are similar to those for $q_{u}$. If $r$ is the concave vertex the proof proceeds similarly. 
Now we will describe the cutting procedure from $p$ to $q_{u}$. Recall from Remark 13 that $w$ is injective on a small neighbourhood of $p$. If we consider $w^{-1}\left(\left[p, q_{u}\right]_{u}\right)$ then there is a unique segment in $D_{b}$ denoted by $I$ whose start point is $w^{-1}(p)=-1$. By definition of $q_{u}$, the segment $\left[p, q_{u}+\varepsilon\right]_{u}$ leaves $w\left(D_{b}\right)$ through $q_{u}$ for $\varepsilon>0$. Thus there is $\tilde{q} \in w^{-1}\left(q_{u}\right)$ which has to be the endpoint of $I$. In fact, since $q_{u}$ lies per definitionem on a boundary segment parting the interior from the exterior $w$ is injective in a neighbourhood of $q_{u}$ such that $\{\tilde{q}\}=w^{-1}\left(q_{u}\right)$ is even unique.

We now cut $D_{b}$ along $I$ into $D_{b}^{v}$ and $D_{b}^{\hat{v}}$ The boundary conditions of $D_{b}^{v}$ are $B_{u}^{v}=I$ and $B_{s}^{v}$ is the segment from -1 to $\tilde{q}$ in $B_{s}$. And for $D_{b}^{\hat{v}}$ we have $B_{u}^{\hat{v}}=I \cup B_{u}$ and $B_{s}^{\hat{v}}$ is the segment from $\tilde{q}$ to 1 in $B_{s}$. Identify $D_{b}^{v}$ and $D_{b}^{\hat{v}}$ with the di-gon $D$ via $h^{v}: D_{b}^{v} \rightarrow D$ with $h^{v}\left(B_{i}^{v}\right)=B_{i}^{D}$ and $h^{\hat{v}}: D_{b}^{\hat{v}} \rightarrow D$ with $h^{\hat{v}}\left(b_{i}^{\hat{v}}\right)=B_{i}^{D}$ for $i \in\{s, u\}$ and define

$$
\begin{array}{ll}
v: D \rightarrow M, & v(z):=w\left(\left(h^{v}\right)^{-1}(z)\right), \\
\hat{v}: D \rightarrow M, & \hat{v}(z):=w\left(\left(h^{\hat{v}}\right)^{-1}(z)\right) .
\end{array}
$$

Since our techniques considered the branches of the (un)stable manifolds separately the $W$-orientation reversing case is reduced to the $W$-orientation preserving case by considering the $W$-orientation preserving $\varphi^{2}$ instead of $\varphi$.

\section{Primary Floer homology}

Set $p^{n}:=\varphi^{n}(p)$ for $p \in \mathcal{H}$ and $n \in \mathbb{Z}$. Keep in mind that in this notation $p=p^{0}$.

3.1. Primary homoclinic points. This subsection introduces a very special kind of homoclinic points, so-called (semi-)primary points. These points are characterized by a very rigid geometric condition. Semi-primary points are usually the first type of homoclinic points investigated by physicists and they play a crucial role in the Melnikov method (see for example RomKedar [RK1, RK2]).

$p \in \mathcal{H} \backslash\{x\}$ is called semi-primary if $] x, p[u \cap] x, p\left[s=\emptyset . p \in \mathcal{H}_{[x]} \backslash\{x\}\right.$ is primary if $] x, p\left[{ }_{u} \cap\right] x, p\left[{ }_{s} \cap \mathcal{H}_{[x]}=\emptyset\right.$. Nonprimary points are called secondary. Clearly, iterates of a (semi-)primary point are again (semi-)primary. If $W^{u} \cap W^{s} \neq \emptyset$ then semi-primary points always exist. We require $[p]=[x]$ in the definition of primary points, since this condition was already necessary for the invariance of the Maslov index and the homotopy classes under the $\mathbb{Z}$-action of $\varphi$. The condition ' $\ldots \cap \mathcal{H}_{[x]}$ ' will be necessary in the invariance discussion.

Remark 16. (1) Let $\varphi$ be $W$-orientation preserving, $p \in \mathcal{H}$ (semi-) primary and denote the branches containing $p$ by $W_{p}^{u}$ and $W_{p}^{s}$. Then for every 
(semi-)primary $q \in\left(W_{p}^{u} \cap W_{p}^{s}\right) /\left\{p^{n} \mid n \in \mathbb{Z}\right\}$ there is a unique $n \in \mathbb{Z}$ such that $\left.q^{n} \in\right] p, p^{1}\left[{ }_{u} \cap\right] p, p^{1}\left[{ }_{s}\right.$.

If $\varphi$ is $W$-orientation reversing then $p^{1}$ has to be replaced by $p^{2}$ and $n$ by $2 n$.

(2) Let $p$ be semi-primary and $q$ primary within the same pair of branches. If $q \in] p, p^{1}\left[{ }_{u}\right.$ then $\left.q \notin\right] x, p^{1}\left[{ }_{s}\right.$. If $\left.q \in\right] p, p^{1}\left[{ }_{s}\right.$ then $\left.q \notin\right] x, p\left[{ }_{u}\right.$. Moreover there is $k \in \mathbb{N}_{0}, n \in \mathbb{Z}$ such that $\left.\left.q \in\right] p^{n}, p^{n+1}{ }_{[u} \cap\right] p^{n+k}, p^{n+k+1}{ }_{[s}$. There are schematic tangles with $k>0$.

Now consider the universal covering $\tau:(\tilde{M}, \tilde{\omega}) \rightarrow(M, \omega)$ with $\tilde{\omega}=\tau^{*} \omega$. For $\tilde{x} \in \tau^{-1}(x)$ and $i \in\{s, u\}$, denote by $\tilde{W}^{i}(\tilde{x})$ the lift of $W^{i}$ passing through $\tilde{x}$. We denote the lift of the segment $[p, q]_{i}$ starting in $\tilde{p} \in \tau^{-1}(p)$ and ending in $\tilde{q} \in \tau^{-1}(q)$ by $[\tilde{p}, \tilde{q}]_{i}$. Given $\tilde{x}_{u}, \tilde{x}_{s} \in \tau^{-1}(x)$, we call $\tilde{p} \in$ $\tilde{W}^{u}\left(\tilde{x}_{u}\right) \cap \tilde{W}^{s}\left(\tilde{x}_{s}\right)$ homoclinic if $\tilde{x}_{u}=\tilde{x}_{s}$ and otherwise heteroclinic. Lifting the tangle (to $\tilde{x} \in \tau^{-1}(x)$ ) means that we consider the tangle generated by $\tilde{W}^{i}:=\tilde{W}^{i}(\tilde{x})$ for $i \in\{s, u\}$ on $\tilde{M}$. With a contractible $p \in W^{u} \cap W^{s}$, we associate $\tilde{p} \in \tau^{-1}(p)$ such that the lift of $[p, x]_{i}$ starting in $\tilde{p}$ ends in $\tilde{x}$. With a noncontractible $p$, we associate $\tilde{p}$ such that the lift of $[p, x]_{u}$ starting in $\tilde{p}$ ends in $\tilde{x}=\tilde{x}_{u}$. Therefore contractible homoclinic points lift to homoclinic points and noncontractible ones to heteroclinic ones.

$\tilde{p} \in \tilde{W}^{u}(\tilde{x}) \cap \tilde{W}^{s}(\tilde{x})$ is called primary if $] \tilde{p}, \tilde{x}\left[_{u} \cap\right] \tilde{p}, \tilde{x}\left[_{s}=\emptyset\right.$. For $\tilde{p}, \tilde{q}, \tilde{r} \in$ $\tilde{W}^{u} \cap \tilde{W}^{s}$, we define $\mathcal{M}(\tilde{p}, \tilde{q}), \widehat{\mathcal{M}}(\tilde{p}, \tilde{q}), \mathcal{N}(\tilde{p}, \tilde{r})$ and $\widehat{\mathcal{N}}(\tilde{p}, \tilde{r})$ in the obvious way.

Now consider the tangle lifted to $\tilde{x} \in \tau^{-1}(x) . p \in W^{u} \cap W^{s}$ is primary if and only if $\tilde{p} \in \tilde{W}^{u} \cap \tilde{W}^{s}$ is primary. Moreover, Remark 16 holds also for the primary points in $\tilde{W}^{u} \cap \tilde{W}^{s}$. The following statements are independent of the chosen reference point $x$.

Lemma 17. Let $\tilde{p} \in \tilde{W}^{u} \cap \tilde{W}^{s}$ be primary. Then $\mu(\tilde{p}):=\mu(\tilde{p}, \tilde{x}) \in$ $\{ \pm 1, \pm 2, \pm 3\}$. There is either an embedded di-gon or an embedded heart or an embedded 2-gons with two concave vertices from $\tilde{p}$ to $\tilde{x}$ (resp. from $\tilde{x}$ to $\tilde{p}$ depending on the sign of the index). For the primary $p:=\tau(\tilde{p})$ follows $\mu(p):=\mu(p, x) \in\{ \pm 1, \pm 2, \pm 3\}$.

Proof. Since $[\tilde{p}]=[\tilde{x}]$, the two points can be connected by a path in $\mathcal{P}\left(W^{u}, W^{s}\right)$. Since $] \tilde{p}, x\left[_{u} \cap\right] \tilde{p}, x\left[_{s}=\emptyset\right.$ the region enclosed by $[\tilde{p}, \tilde{x}]_{u}$ and $[\tilde{p}, \tilde{x}]_{s}$ is an embedded polygon with two vertices. Assume the intersections in $\tilde{p}$ and $\tilde{x}$ to be orthogonal and parametrize the segments $[\tilde{p}, \tilde{x}]_{u}$ from $\tilde{p}$ to $\tilde{x}$ and $[\tilde{p}, \tilde{x}]_{s}$ from $\tilde{x}$ to $\tilde{p}$. Then the Maslov index is twice the winding number of the tangent vector of the segments. Thus only $\mu(\tilde{p}, \tilde{x}) \in\{ \pm 1, \pm 2, \pm 3\}$ can be realized without violating the boundary condition $] \tilde{p}, \tilde{x}\left[{ }_{u} \cap\right] \tilde{p}, \tilde{x}\left[_{s}=\emptyset\right.$. Therefore $\mu(p)=\mu(\tilde{p}) \in\{ \pm 1, \pm 2, \pm 3\}$ for $p=\tau(\tilde{p})$.

Note that the immersion between a primary $p$ and the fixed point $x$ does not need to be globally injective since there might be noncontractible points in $] x, p[u \cap] x, p[s$. 
Remark 18. (1) Let $i \in\{s, u\}, \tilde{p} \in \tilde{W}^{u} \cap \tilde{W}^{s}$ be primary and $p:=\tau(\tilde{p})$. Lift $\gamma_{i}$ to $\tilde{\gamma}_{i}: \mathbb{R} \rightarrow \tilde{W}^{i}$ and obtain the ordering $<_{i}$ on $\tilde{W}^{i}$. W.l.o.g. assume $p<_{i} \varphi(p)$; for $W$-orientation reversing $\varphi$ use $p<_{i} \varphi^{2}(p)$. Then

$$
\begin{aligned}
& \tilde{p}_{+}:=\max \left\{\tilde{q} \in \tilde{W}^{s} \mid \tilde{q}<_{s} \tilde{p}, \tilde{q} \in\right] \tilde{x}, \tilde{p}\left[{ }_{u}\right\}, \\
& \tilde{p}_{-}:=\min \left\{\tilde{q} \in \tilde{W}^{u} \mid \tilde{p}<_{u} \tilde{q}, \tilde{q} \in\right] \tilde{x}, \tilde{p}\left[\left[_{s}\right\}\right.
\end{aligned}
$$

are primary and $\tilde{p}_{ \pm}$is called adjacent to $\tilde{p}$.

(2) Let $\tilde{p}$ be primary and $\tilde{q}=\tilde{p}_{ \pm}$. Then $] \tilde{p}, \tilde{q}[u \cap] \tilde{p}, \tilde{q}\left[{ }_{s}=\emptyset\right.$. If moreover $\tilde{p}$ and $\tilde{q}$ are transverse then $\mu(\tilde{p}, \tilde{q}) \in\{1,-1\}$ and there is an embedded di-gon between them.

(3) transverse primary points in $\left.] \tilde{p}, \tilde{p}^{1}{ }_{u} \cap\right] \tilde{p}, \tilde{p}^{1}{ }_{s}$. Let $\tilde{p}$ be primary and order the primary points in $\left[\tilde{p}, \tilde{p}^{-1}\right]_{u} \cap\left[\tilde{p}, \tilde{p}^{-1}\right]_{s}$ via $\tilde{p}, \tilde{p}_{+}$, $\left(\tilde{p}_{+}\right)_{+}, \ldots, \tilde{p}^{-1}$ and assume them transverse. Then their relative Maslov index alternates between +1 and -1 .

(4) Let all primary points $p \in W^{u} \cap W^{s}$ be transverse. Then there are modulo $\mathbb{Z}$-action only finitely many primary points. The same is true for the primary points in $\tilde{W}^{u} \cap \tilde{W}^{s}$.

3.2. Signs and coherent orientations. Now we define the signs needed for the definition of the boundary operator of the Floer chain complex. The signs have to satisfy a certain compatibility with the cutting and gluing procedure which is known in classical Floer theory as 'coherent orientations'.

We will define two kinds of signs with slightly different properties depending on the type of homoclinic points (primary or secondary) and the symplectomorphism ( $W$-orientation preserving or reversing).

For $i \in\{s, u\}$, associate to each branch $W_{+}^{i}$ and $W_{-}^{i}$ its 'jump direction' as orientation and denote it by $o\left(W_{+}^{i}\right)$ resp. $o\left(W_{-}^{i}\right)$. Let $p, q$ be primary with $\mu(p, q)=1$ and $v \in \mathcal{M}(p, q)$. Associate to $v\left(B_{i}\right)=[p, q]_{i}$ the orientation induced by the parametrization from $p$ to $q$ and call it $o_{p q}$. In (the proof of) Proposition 26, we will show that $x \notin] p, q[u \cap] p, q[s$. Thus, there is a branch $W_{p q} \in\left\{W_{+}^{u}, W_{-}^{u}, W_{+}^{s}, W_{-}^{s}\right\}$ containing both $p$ and $q$. We set

$$
m(p, q):=\left\{\begin{array}{llll}
1 & \text { if } \mu(p, q)=1, & \mathcal{M}(p, q) \neq \emptyset, & o\left(W_{p q}\right)=o_{p q}, \\
-1 & \text { if } \mu(p, q)=1, & \mathcal{M}(p, q) \neq \emptyset, & o\left(W_{p q}\right) \neq o_{p q} \\
0 & \text { otherwise. } & &
\end{array}\right.
$$

If there are two branches $W_{p q}^{u}$ and $W_{p q}^{s}$ containing $p$ and $q$ then $p$ and $q$ are adjacent and $o\left(W_{p q}^{u}\right)=o_{p q}=o\left(W_{p q}^{s}\right)$, compare Figure 4. Thus $m(p, q)$ is well defined. We do not need to distinguish the cases $W$-orientation preserving and reversing since $m(p, q)=m\left(p^{l}, q^{l}\right)$ for all $l \in \mathbb{Z}$. The definition does not generalize to arbitrary homoclinic points.

Lemma 19. Let $p$ and $r$ be primary with $\mu(p, r)=2$ and $w \in \widehat{\mathcal{N}}(p, r)$. For $i \in\{s, u\}$ assume the existence of $q_{i}$ with $\mu\left(p, q_{i}\right)=1=\mu\left(q_{i}, r\right)$ and 
(i) Case $] x, p\left[{ }_{u} \cap\right] x, q\left[{ }_{u}=\emptyset \neq\right] x, p\left[\left[_{s} \cap\right] x, q[s\right.$ Subcase $] x, p\left[{ }_{s} \subset\right] x, q\left[{ }_{s}\right.$

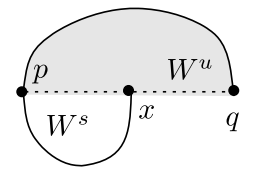

$(-1,2)$

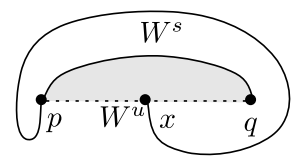

$(3,-2)$

(ii) Case $] x, p\left[{ }_{u} \cap\right] x, q\left[{ }_{u} \neq \emptyset=\right] x, p\left[{ }_{s} \cap\right] x, q\left[{ }_{s}\right.$ Subcase $] x, p\left[{ }_{u} \subset\right] x, q\left[{ }_{u}\right.$

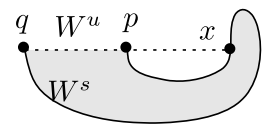

$(3,-2)$

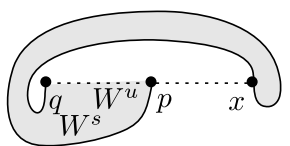

$(3,-2)$

(iii) Case $] x, p\left[{ }_{u} \cap\right] x, q\left[{ }_{u} \neq \emptyset \neq\right] x, p\left[{ }_{u} \cap\right] x, q\left[{ }_{s}\right.$ ]$x, p\left[{ }_{u} \subset\right] x, q\left[{ }_{u},\right] x, q\left[_{s} \subset\right] x, p\left[{ }_{s}\right.$

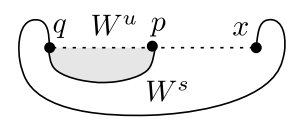

$(-2,3)$

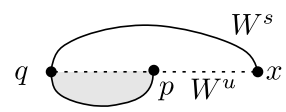

$(2,-1)$

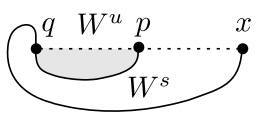

$(-1,2)$

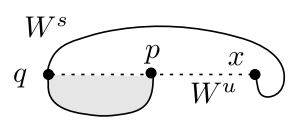

$(3,-2)$

Figure 4. Immersions of relative index 1 up to obvious symmetries.

$v_{i} \in \widehat{\mathcal{M}}\left(p, q_{i}\right)$ and $\hat{v}_{i} \in \widehat{\mathcal{M}}\left(q_{i}, r\right)$ such that $\hat{v}_{i} \# v_{i}=w$. Then

$$
m\left(p, q_{u}\right) \cdot m\left(q_{u}, r\right)=-m\left(p, q_{s}\right) \cdot m\left(q_{s}, r\right) .
$$

Proof. Have a look at Proposition 29 and check in Figure 5 the eight possible $w=\hat{v}_{i} \# v_{i} \in \widehat{\mathcal{N}}(p, r)$ sketched in the left and right column. This yields the claim. 


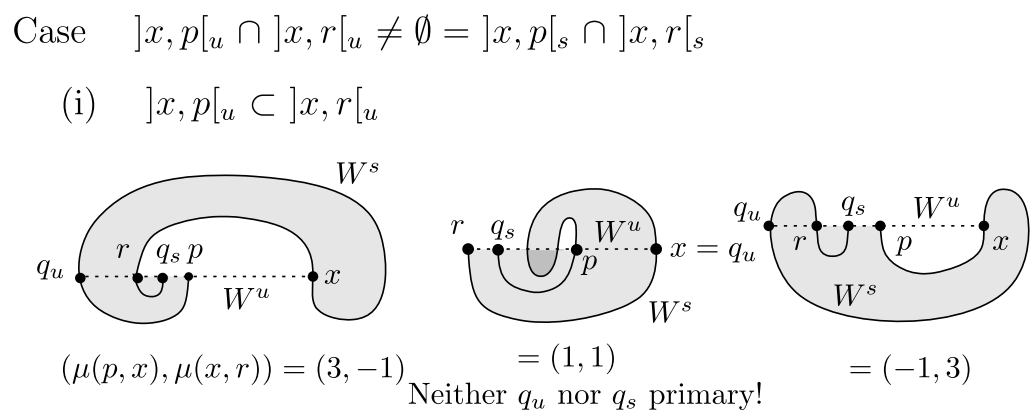

(ii) $\quad] x, r\left[{ }_{u} \subset\right] x, p\left[{ }_{u}\right.$
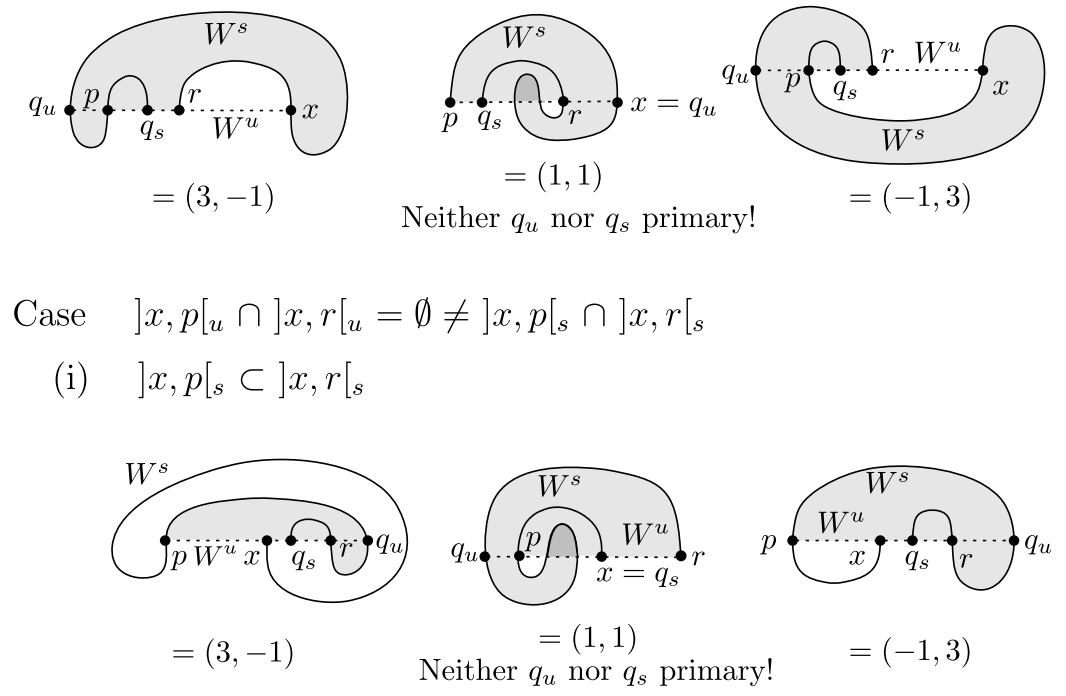

(ii) $\quad] x, r[s \subset] x, p[s$
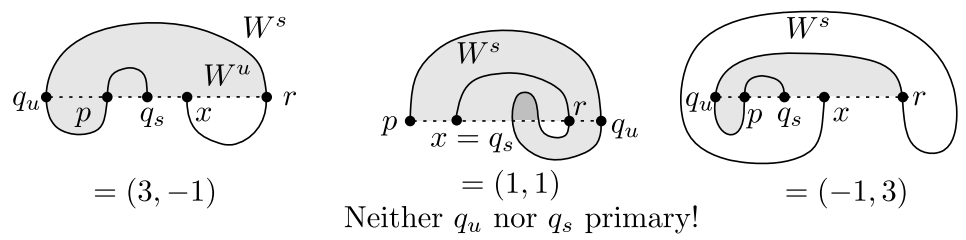

Figure 5. Cutting for primary points.

Whereas $m(p, q)$ is well defined only for primary points, there is another way to define signs for arbitrary homoclinic points: Fix an orientation $o_{u}$ on $W^{u}$. Now let $p, q \in \mathcal{H}$ with $\mu(p, q)=1$ and provide $[p, q]_{u}$ with the orientation $o_{p q}$ induced by the parametrization from $p$ to $q$. For $W$-orientation 
preserving $\varphi$, we define

$$
n(p, q):= \begin{cases}+1 & \text { if } \widehat{\mathcal{M}}(p, q) \neq \emptyset \text { and } o_{p q}=o_{u} \\ -1 & \text { if } \widehat{\mathcal{M}}(p, q) \neq \emptyset \text { and } o_{p q} \neq o_{u} \\ 0 & \text { if } \widehat{\mathcal{M}}(p, q)=\emptyset\end{cases}
$$

$n(p, q)$ clearly also could be defined using an orientation on $W^{s}$. For $W$ orientation reversing $\varphi$ we have to set $n_{2}(p, q):=n(p, q) \bmod 2$. The signs depend as follows from the chosen data: Set $\mathcal{H}_{[x]}^{l}=\{p \in \mathcal{H} \mid \mu(p, x)=$ $l,[p]=[x]\}$ and provide $W^{i}$ with the orientation induced by $\dot{\gamma}_{i}$. Let $\sigma_{01}:=$ $\operatorname{sign}\left(\operatorname{det}\left(\dot{\gamma}_{u}(0), \dot{\gamma}_{s}(0)\right)\right)$ and denote the signs defined via the orientation on $W^{i}$ by $n\left(p, q, W^{i}\right)$. Then

$$
\begin{aligned}
& n\left(p, q, W^{u}\right)=\sigma_{01} n\left(p, q, W^{s}\right), \quad \text { for } p \in \mathcal{H}_{[x]}^{2 l}, \\
& n\left(p, q, W^{u}\right)=-\sigma_{01} n\left(p, q, W^{s}\right), \quad \text { for } p \in \mathcal{H}_{[x]}^{2 l+1}
\end{aligned}
$$

for all $q \in \mathcal{H}_{[x]}$ and $l \in \mathbb{Z}$.

Lemma 21. Let $p, r \in \mathcal{H}$ with $\mu(p, r)=2$ and $w \in \mathcal{N}(p, r)$. For $i \in\{s, u\}$ consider $q_{i} \in \mathcal{H}$ with $\mu\left(p, q_{i}\right)=1=\mu\left(q_{i}, r\right)$ and $\hat{v}_{i} \in \mathcal{M}\left(p, q_{i}\right)$ and $v_{i} \in$ $\mathcal{M}\left(q_{i}, r\right)$ such that $\hat{v}_{i} \# v_{i}=w$. Then

$$
n\left(p, q_{u}\right) \cdot n\left(q_{u}, r\right)=-n\left(p, q_{s}\right) \cdot n\left(q_{s}, r\right)
$$

and this relation also is true for $n_{2}$.

Proof. Consider Figure 2, choose an orientation on $W^{u}$ and check that the claim is true. If we choose the other orientation on $W^{u}$ all signs swap and the relation remains true.

3.3. Primary Floer homology. Now we are ready to define the Floer chain complex. We assume from now on (if not stated otherwise) all homoclinic points to be primary and transverse.

We define on $\mathcal{H}_{p r}:=\{p \in \mathcal{H} \mid p$ primary $\}$ an equivalence relation via $p \sim q \Leftrightarrow \exists n \in \mathbb{Z}$ with $q^{n}=p$. We set $\tilde{\mathcal{H}}_{p r}:=\mathcal{H}_{p r} / \sim$ and denote by $\langle p\rangle$ the equivalence class of $p$. Note that $\# \tilde{\mathcal{H}}_{p r}<\infty$ according to Remark 18. Owing to Remark 7, we can establish a well-defined homotopy class and a Maslov index via $[\langle p\rangle]:=[p], \mu(\langle p\rangle,\langle q\rangle):=\mu(p, q)$ and $\mu(\langle p\rangle):=\mu(p, x)$. We define

$$
\begin{gathered}
\mathfrak{C}_{m}:=\mathfrak{C}_{m}(x, \varphi ; \mathbb{Z}):=\bigoplus_{\substack{p \in \mathcal{H}_{p r} \\
\mu(p)=m}} \mathbb{Z} p, \\
\mathfrak{d}_{m}: \mathfrak{C}_{m} \rightarrow \mathfrak{C}_{m-1}, \quad \mathfrak{d}(p)=\sum_{\substack{q \in \mathcal{H}_{p r} \\
\mu(q)=\mu(p)-1}} m(p, q) q
\end{gathered}
$$


on a generator $p$ and extend $\mathfrak{d}$ by linearity. $\varphi$ induces $\varphi_{*}: \mathfrak{C}_{*} \rightarrow \mathfrak{C}_{*}$ satisfying $\varphi_{*} \circ \mathfrak{d}=\mathfrak{d} \circ \varphi_{*}$. The sum is finite since $\# \tilde{\mathcal{H}}_{p r}<\infty$ and, as we will see later

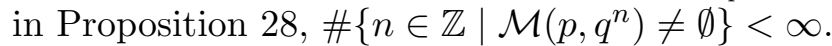

$\mu(p)=\mu\left(p^{n}\right)$ for $n \in \mathbb{Z}$ implies that the chain groups have infinite rank over $\mathbb{Z}$. However, since $\mu(p):=\mu(p, x) \in\{ \pm 1, \pm 2, \pm 3\}$ for $p \in \mathcal{H}_{p r}$ there are at most six nonvanishing chain groups.

Theorem 22. $\mathfrak{d} \circ \mathfrak{d}=0$, i.e., $\left(\mathfrak{C}_{*}, \mathfrak{d}_{*}\right)$ is a chain complex.

The proof of Theorem 22 is postponed to the following subsections. The homology of $\left(\mathfrak{C}_{*}, \mathfrak{d}\right)$ is

$$
\mathfrak{H}_{m}:=\mathfrak{H}_{m}(x, \varphi ; \mathbb{Z}):=\frac{\operatorname{ker} \mathfrak{d}_{m}}{\operatorname{Im} \mathfrak{d}_{m+1}} .
$$

Since the chain groups have infinite rank over $\mathbb{Z}$ this might also be the case for the homology groups. In order to enforce finite rank, we will divide by the $\mathbb{Z}$-action: For $\langle p\rangle,\langle q\rangle \in \tilde{\mathcal{H}}_{p r}$ set $m(\langle p\rangle,\langle q\rangle):=\sum_{n \in \mathbb{Z}} m\left(p, q^{n}\right)$ and define

$$
\begin{gathered}
C_{m}:=C_{m}(x, \varphi ; \mathbb{Z}):=\bigoplus_{\substack{\langle p\rangle \tilde{\mathcal{H}}_{p r} \\
\mu(\langle p\rangle)=m}} \mathbb{Z}\langle p\rangle, \\
\partial_{m}: C_{m} \rightarrow C_{m-1}, \quad \partial\langle p\rangle:=\sum_{\substack{\langle q\rangle \in \tilde{\mathcal{H}}_{p r} \\
\mu(\langle q\rangle)=\mu(\langle p\rangle)-1}} m(\langle p\rangle,\langle q\rangle)\langle q\rangle
\end{gathered}
$$

on a generator $\langle p\rangle$ and extend $\partial$ by linearity. The compatibility of the $\mathbb{Z}$ action with the Maslov index and the homotopy classes yields the welldefinedness of $\partial$.

We have $\operatorname{rk}_{\mathbb{Z}}\left(C_{m}\right)=\#\left\{\langle p\rangle \in \tilde{\mathcal{H}}_{p r} \mid \mu(\langle p\rangle)=m\right\}<\infty$. And due to Lemma 17, at most $C_{ \pm 1}, C_{ \pm 2}$ and $C_{ \pm 3}$ are nonzero. Moreover, Remark 18 implies $\mathrm{rk}_{\mathbb{Z}} C_{ \pm 2}=\mathrm{rk}_{\mathbb{Z}} C_{ \pm 1}+\mathrm{rk}_{\mathbb{Z}} C_{ \pm 3}$.

If we generalize the notion of equivalence classes to finite sums via $\langle p+q\rangle=$ $\langle p\rangle+\langle q\rangle$ the differential can also be written as

$$
\partial\langle p\rangle=\langle\mathfrak{d} p\rangle=\sum_{\substack{q \in \mathcal{H}_{p r} \\ \mu(q)=\mu(p)-1}} m(p, q)\langle q\rangle .
$$

Therefore $\mathfrak{d}^{2}=0$ immediately implies

Theorem 23. $\partial \circ \partial=0$, i.e., $\left(C_{*}, \partial_{*}\right)$ is a chain complex.

We define the primary Floer homology of $\varphi$ in $x$ as

$$
H_{m}:=H_{m}(x, \varphi ; \mathbb{Z}):=\frac{\operatorname{ker} \partial_{m}}{\operatorname{Im} \partial_{m+1}}
$$

Since already the $C_{m}$ have finite rank over $\mathbb{Z}$ so has $H_{m}$. All chain groups $C_{m}$ and homology groups $H_{m}$ with $m \neq \pm 1, \pm 2, \pm 3$ vanish. 
Homology and Cohomology. The question about cohomology instead of homology leads in our situation to the choice between $\varphi$ and $\varphi^{-1}$ as underlying symplectomorphism. More precisely, $H_{*}(x, \varphi)$ is related to $H_{*}\left(x, \varphi^{-1}\right)$ in the following way. Consider

$$
C^{m}(x, \varphi ; \mathbb{Z}):=\bigoplus_{\substack{\langle p\rangle \in \tilde{\mathcal{H}}_{p r} \\ \mu(\langle p\rangle)=m}} \mathbb{Z}\langle p\rangle
$$

with differential $\delta: C^{m}(x, \varphi ; \mathbb{Z}) \rightarrow C^{m+1}(x, \varphi ; \mathbb{Z})$ defined on the generators by

$$
\delta(\langle p\rangle):=\sum_{\substack{q \in \mathcal{H}_{p r} \\ \mu(q)=m+1}} m(q, p)\langle q\rangle .
$$

Then $\delta \circ \delta=0$ is proven analogously to $\partial \circ \partial=0$ and

$$
H^{*}(x, \varphi ; \mathbb{Z}):=\frac{\operatorname{ker} \delta}{\operatorname{Im} \delta}
$$

is called primary Floer cohomology of $\varphi$ in $x$. Changing from $\varphi$ to $\varphi^{-1}$ transforms $W^{u}$ into $W^{s}$ and vice versa, but apart from this the homoclinic tangle remains untouched. Therefore the sign of the Maslov index of a homoclinic point $p=p_{\varphi}$ in the tangle generated by $\varphi$ changes, when considered as homoclinic point $p=p_{\varphi^{-1}}$ in the tangle corresponding to $\varphi^{-1}$, i.e., $\mu\left(p_{\varphi}\right)=-\mu\left(p_{\varphi^{-1}}\right)$. This implies

Theorem 25. $H^{*}(x, \varphi)=H_{-*}\left(x, \varphi^{-1}\right)$.

The signs $\boldsymbol{n}(\boldsymbol{p}, \boldsymbol{q})$. The above chain complexes and homologies can be defined analogously with $n(p, q)$ resp. $n_{2}(p, q)$ (and $\mathbb{Z} / 2 \mathbb{Z}$-coefficients in the latter case). We will see that $\mathfrak{H}_{m}$ and $H_{m}$ do not depend on the chosen data: Let $\varphi$ be $W$-orientation preserving. Changing the orientation of $W^{u}$ changes the sign of the $n(p, q)$. Thus $\mathfrak{d}$ transforms into $-\mathfrak{d}$ which has the same kernel and image as $\mathfrak{d}$. (20) implies that the differential obtained by using an orientation on $W^{s}$ instead of $W^{u}$ equals for fixed Maslov index \pm 1 times the $W^{u}$-induced differential. Thus ker $\mathfrak{d}_{k}^{W^{u}}=\operatorname{ker} \mathfrak{d}_{k}^{W^{s}}$ and $\operatorname{Im} \mathfrak{d}_{k}^{W^{u}}=\operatorname{Im} \mathfrak{d}_{k}^{W^{s}}$ for all $k$ such that the homologies coincide. $H_{m}$ does not depend on the choice of the orientation for the same reasons as $\mathfrak{H}_{m}$.

If $\varphi$ is $W$-orientation reversing we have to use $\mathbb{Z} / 2 \mathbb{Z}$-coefficients $n_{2}(p, q)$ if we want to be able to divide by the $\mathbb{Z}$-action.

If one computes the examples in Chapter 4 with $n(p, q)$ instead with $m(p, q)$ one obtains isomorphic homologies, but the generators of the homology groups differ.

3.4. Well-definedness, gluing and cutting. In this subsection, we will prove Theorem 22 . The proof is mainly based on classifications of immersions of relative Maslov index 1 and 2 . 
Lift the homoclinic tangle w.r.t. $\tilde{x} \in \tau^{-1}(x)$. Given primary $p, q \in W^{u} \cap$ $W^{s}$ with associated primary $\tilde{p}, \tilde{q} \in \tilde{W}^{u} \cap \tilde{W}^{s}$, the immersions in $\mathcal{M}(p, q)$ resp. $\mathcal{N}(p, q)$ lift exactly to the immersions in $\mathcal{M}(\tilde{p}, \tilde{q})$ resp. $\mathcal{N}(\tilde{p}, \tilde{q})$. Primary Floer (co)homology is well-defined for $(\varphi, x)$ on $M$ if and only if it is welldefined for the lifted homoclinic tangle generated by $\tilde{W}^{u}$ and $\tilde{W}^{s}$ on $\tilde{M}$. Thus it is enough to prove the primary cutting and gluing procedure for the lifted tangle $\tilde{W}^{u} \cap \tilde{W}^{s}$ on $\tilde{M}$.

Proposition 26 (Classification for index difference 1). Let $p, q \in \mathcal{H}$ be primary with $\mu(p, q)=1$ and let $\tilde{p}$ and $\tilde{q}$ the associated primary points in $\tilde{W}^{u} \cap \tilde{W}^{s}$. Then either $\mathcal{M}(\tilde{p}, \tilde{q})=\emptyset$ or $v \in \mathcal{M}(\tilde{p}, \tilde{q})$ is, in fact, an embedding.

The elements of $\mathcal{M}(p, q)$ do not need to be embeddings. Nor is it true for noncontractible semi-primary points.

Proof. In the following, we work with the lifted tangle on $\tilde{M}$. We drop the tilde associated to symbols on $\tilde{M}$. Thus identify $p=\tilde{p}$ and $q=\tilde{q}$ etc.

The proof is tedious, but simple. $[p]=[q]=[x]$ allows us to write $1=\mu(p, q)=\mu(p, x)+\mu(x, q)$ and Lemma 17 provides the four cases $(\mu(p, x), \mu(x, q)) \in\{(-2,3),(-1,2),(2,-1),(3,-2)\}$. Since there are always two possibilities to place the concave vertex of a standard heart the number of cases multiplies by two. Moreover, we have to distinguish $] x, p[i \cap] x, q[i=$ $\emptyset$ or $\neq \emptyset$ for $i \in\{s, u\}$. Since $W^{i}$ is self-intersection free and one-dimensional we conclude in case $] x, p[i \cap] x, q\left[i \neq \emptyset\right.$ either $[x, p]_{i} \subset[x, q]_{i}$ or $[x, q]_{i} \subset[x, p]_{i}$. This yields a lot of cases, but fortunately some of them are symmetric. We recall from Lemma 17 that there is modulo parametrization exactly one embedding between $p$ and $x$ and $q$ and $x$. Since embeddings are by definition bijective there is - together with the boundary conditions - almost no degree of freedom in sketching them. Figure 4 lists all possibly arising immersions.

Since, according to Proposition 26, immersions between primary homoclinic points $\tilde{p}$ and $\tilde{q}$ of $\tilde{W}^{u} \cap \tilde{W}^{s}$ are in fact embeddings it is enough to show ]$\tilde{p}, \tilde{q}[u \cap] \tilde{p}, \tilde{q}[s \neq \emptyset$ to prevent their existence:

Lemma 27. Let $p, q \in \mathcal{H}_{[x]} \backslash\{x\}$ and $p^{n}:=\varphi^{n}(p)$ etc. for $n \in \mathbb{Z}$. Let $\tilde{p}$, $\tilde{q}$ and $\tilde{p}^{n}$ etc. be the associated points in $\tilde{W}^{u} \cap \tilde{W}^{s}$. Then there is $N \in \mathbb{N}_{0}$ such that for $n \in \mathbb{Z}$ with $|n| \geq N$ we have $] \tilde{p}, \tilde{q}^{n}\left[{ }_{u} \cap\right] \tilde{p}, \tilde{q}^{n}{ }_{s} \neq \emptyset$.

Proof. Let $\tilde{p}$ etc. be the point associated to $p$ in the lifted tangle on $\tilde{M}$. Let $\varphi$ be $W$-orientation preserving.

Consider the case $x \notin] p, q[u$ and $x \notin] p, q\left[s\right.$. Then there is $N \in \mathbb{N}_{0}$ such that $\left.\tilde{p}^{1} \in\right] \tilde{p}, \tilde{q}^{n}\left[{ }_{u} \cap\right] \tilde{p}, \tilde{q}^{n}\left[{ }_{s}\right.$ for all $n \geq N$ and $\left.\tilde{p}^{-1} \in\right] \tilde{p}, \tilde{q}^{n}\left[{ }_{u} \cap\right] \tilde{p}, \tilde{q}^{n}{ }_{s}$ for all $n \leq-N$.

If $x \in] p, q[u \cap] p, q\left[{ }_{s}\right.$ then $\left.\tilde{x} \in\right] \tilde{p}, \tilde{q}^{n}\left[{ }_{u} \cap\right] \tilde{p}, \tilde{q}^{n}\left[{ }_{s}\right.$ for all $n \in \mathbb{Z}$. 
Consider the case $x \in] p, q[u$ and $x \notin] p, q\left[s\right.$. Then there is $N \in \mathbb{N}_{0}$ such that $\left.\left.\tilde{q}^{N-1} \in\right] \tilde{p}, \tilde{q}^{n}{ }_{u} \cap\right] \tilde{p}, \tilde{q}^{n}{ }_{s}$ for all $n \geq N$ and $\left.\tilde{p}^{-1} \in\right] \tilde{p}, \tilde{q}^{n}\left[{ }_{u} \cap\right] \tilde{p}, \tilde{q}^{n}{ }_{s}$ for all $n \leq-N$. In the case $x \notin] p, q[u$ and $x \in] p, q[$ s conclude analogously.

Now consider $W$-orientation reversing $\varphi$. Here we have to distinguish between even and odd $n \in \mathbb{Z}$. Since $\varphi^{2}$ is orientation preserving the above proof carries over for even $n$ if we replace $p^{1}$ by $p^{2}$ etc. Thus we only have to prove the claim for odd $n$.

If $x \notin] p, q\left[{ }_{u},\right] p, q\left[{ }_{s}\right.$ then $\left.\tilde{x} \in\right] \tilde{p}, \tilde{q}^{n}\left[{ }_{u} \cap\right] \tilde{p}, \tilde{q}^{n}{ }_{s}$ for all odd $n$.

If $x \in] p, q\left[{ }_{u} \cap\right] p, q\left[{ }_{s}\right.$ there is $N \in \mathbb{N}_{0}$ such that $\left.\tilde{p}^{2} \in\right] \tilde{p}, \tilde{q}^{n}\left[{ }_{u} \cap\right] \tilde{p}, \tilde{q}^{n}{ }_{s}$ for all odd $n \geq N$ and $\left.\tilde{p}^{-2} \in\right] \tilde{p}, \tilde{q}^{n}[u \cap] \tilde{p}, \tilde{q}^{n}{ }_{s}$ for all odd $n \leq-N$.

If $x \in] p, q[u$ and $x \notin] p, q\left[s\right.$ then there is an odd $N \in \mathbb{N}_{0}$ such that $\left.\left.\tilde{p}^{2} \in\right] \tilde{p}, \tilde{q}^{n}{ }_{[u} \cap\right] \tilde{p}, \tilde{q}^{n}{ }_{s}$ for odd $n \geq N$ and $\left.\tilde{q}^{N+2} \in\right] \tilde{p}, \tilde{q}^{n}\left[{ }_{u} \cap\right] \tilde{p}, \tilde{q}^{n}{ }_{[s}$ for odd $n \leq-N$.

If $x \notin] p, q[u$ and $x \in] p, q\left[{ }_{s}\right.$ conclude analogously.

Now we prove that for $p \in \mathcal{H}_{p r}$ the differential $\mathfrak{d}$ does not contain infinitely many iterates $m\left(p, q^{n}\right) q^{n}$ of some primary $q$. This implies the welldefinedness of $\mathfrak{d}$ and $\partial$.

Proposition 28. Let $p, q \in \mathcal{H}_{p r}$ and $\mathcal{M}(p, q) \neq \emptyset$ and set $q^{n}:=\varphi^{n}(q)$ for $n \in \mathbb{Z}$. Then

$$
\#\left\{n \in \mathbb{Z} \mid \mathcal{M}\left(p, q^{n}\right) \neq \emptyset\right\}<\infty .
$$

Proof. Denote by $\tilde{p}, \tilde{q}, \tilde{q}^{n}$ etc. the associated points in $\tilde{W}^{u} \cap \tilde{W}^{s}$ and recall that $v \in \mathcal{M}(p, q)$ exists if and only its lift $\tilde{v} \in \mathcal{M}(\tilde{p}, \tilde{q})$ exists. Lemma 27 yields the existence of some $N>0$ such that $] \tilde{p}, \tilde{q}^{n}\left[{ }_{u} \cap\right] \tilde{p}, \tilde{q}^{n}[s \neq \emptyset$ for all $n \in \mathbb{Z}$ with $|n| \geq N$.

Assume $\tilde{v}_{n} \in \mathcal{M}\left(\tilde{p}, \tilde{q}^{n}\right) \neq \emptyset$ for some $n$ with $|n| \geq N$. Since $\left[\tilde{p}, \tilde{q}^{n}\right]_{u}=$ $\tilde{v}_{n}\left(B_{u}\right)$ and $\left[\tilde{p}, \tilde{q}^{n}\right]_{s}=\tilde{v}_{n}\left(B_{s}\right)$ there is $z_{u} \in B_{u}$ and $z_{s} \in B_{s}$ such that $\tilde{v}_{n}\left(z_{u}\right)=\tilde{v}_{n}\left(z_{s}\right)$. Since $\tilde{W}^{u}$ and $\tilde{W}^{s}$ do not have self-intersections it follows $z_{u}, z_{s} \notin\{(-1,0),(1,0)\}$. Therefore $\tilde{v}_{n}$ is not globally injective and thus no embedding. The claim now follows from Proposition 26.

The gluing theorem for primary points is clearly a special case of Theorem 14. However, it is a priori not clear, that the cutting procedure yields two primary 'cutting points' $q_{u}$ and $q_{s}$.

Proposition 29 (Classification for index difference 2). Let $p, r \in \mathcal{H}_{p r}$ with $\mu(p, r)=2$ and let $\tilde{p}$ and $\tilde{r}$ be the associated points in $\tilde{W}^{u} \cap \tilde{W}^{s}$. The possibly arising immersed hearts $w \in \widehat{\mathcal{N}}(\tilde{p}, \tilde{r})$ appear shadowed in Figure 5. $w$ is an embedding apart from the case $(\mu(\tilde{p}, \tilde{x}), \mu(\tilde{x}, \tilde{r}))=(1,1)$ where it is not globally injective.

Proof. In the following, we work with the lifted tangle on $\tilde{M}$. We drop the tilde associated to symbols on $\tilde{M}$, i.e., we identify $p=\tilde{p}$ and $r=\tilde{r}$ etc. 
Since $[p]=[r]=[x]$ we can write $\mu(p, r)=\mu(p, x)+\mu(x, r)=2$. Now we proceed as in the proof of Proposition 26 and check the possible combinations for $(\mu(p, x), \mu(x, r))$. Lemma 17 restricts the possibilities to $(\mu(p, x), \mu(x, r)) \in\{(3,-1),(1,1),(-1,3)\}$ and we recall that the immersions of index $\mu(p, x)$ and $\mu(x, r)$ between $p$ and $x$ and $x$ and $r$ are embeddings. As before, we will consider the cases $] x, p[i \cap] x, r[i=\emptyset$ or $\neq \emptyset$. If ]$x, p\left[{ }_{i} \cap\right] x, r\left[{ }_{i} \neq \emptyset\right.$ this implies $[x, p]_{i} \subset[x, r]_{i}$ or $[x, r]_{i} \subset[x, p]_{i}$ since $W^{i}$ is free of self-intersections and $\operatorname{dim} W^{i}=1$. All possibly arising immersions are listed in Figure 5.

It will turn out that for primary $p$ and $r$ with $\mu(p, r)=2$ either both cutting points $q_{u}$ and $q_{s}$ are primary or none of them. In the proof of Theorem 15 , strongly intersecting is only needed if the concave vertex of the heart is the fixed point. Since $x \notin \mathcal{H}_{p r}$ we can drop this assumption in the following statement. Moreover, in Theorem 15, the $\lambda$-lemma was applied to the intersection at the concave vertex of the immersion. Thus it is enough to require only the primary points to be transverse.

Theorem 30 (Cutting for primary points). Let all primary points be transverse and $p, r \in \mathcal{H}_{p r}$ with $\mu(p, r)=2$ and $w \in \mathcal{N}(p, r)$. Then there are unique points $q_{u}$ and $q_{s}$ such that either both $q_{i}$ are primary admitting $v_{i} \in \mathcal{M}\left(p, q_{i}\right)$ and $\hat{v}_{i} \in \mathcal{M}\left(q_{i}, r\right)$ with $\hat{v}_{i} \# v_{i}=w$ for $i \in\{s, u\}$ or none of them is primary.

Proof. It is sufficient to show the claim for the lifted tangle generated by $\tilde{W}^{u}$ and $\tilde{W}^{s}$ on $\tilde{M}$. We drop the tilde associated to symbols on $\tilde{M}$ and identify $\tilde{p}=p$ etc.

Let $p$ and $r$ be primary with $\mu(p, r)=2 . p$ and $r$ are transverse intersection points such that the existence (and uniqueness) of the cutting points $q_{u}$ and $q_{s}$ follows from the proof of Theorem 15. However, $q_{u}$ and $q_{s}$ might be nontransverse. We will prove that $q_{u}$ and $q_{s}$ are either both primary or both nonprimary. If both are primary then they are, by assumption, transverse and the claim follows from Theorem 15.

Proposition 29 together with Figure 5 describes all possible immersions of index difference 2 and sketches $q_{u}$ and $q_{s}$ and the cuts to $q_{u}$ and $q_{s}$. For simplicity, the $q_{i}$ are sketched transverse.

Checking the shapes in Figure 5, we find that for all cases $(\mu(p, x), \mu(x, r)) \in$ $\{(3,-1),(-1,3)\}$ the immersion $w$ is an embedding and that $q_{u}$ and $q_{s}$ are both primary. In the case $] x, p\left[{ }_{u} \cap\right] x, r\left[{ }_{u} \neq \emptyset=\right] x, p[s \cap] x, r[s$, we only sketched the case $q_{u} \in[x, p]_{s}$, but also $q_{u} \in[x, r]_{s}$ would be primary. In the case $] x, p\left[{ }_{u} \cap\right] x, r\left[{ }_{u}=\emptyset \neq\right] x, p\left[{ }_{s} \cap\right] x, r\left[{ }_{s}\right.$, we have to distinguish $q_{s} \in[x, p]_{u}$ or $q_{s} \in[x, r]_{u}$, but in both cases $q_{s}$ is primary.

Now consider the case $(\mu(p, x), \mu(x, r))=(1,1)$. First, we note that $w$ is not necessarily an embedding. One of the cutting points is the fixed point which is per definitionem not primary. Furthermore, those segments which 
join the other cutting point to $x$ overcross in $p$ or $r$ such that this cutting point also is nonprimary.

Proof of Theorem 22. In the following, we work with the lifted tangle on $\tilde{M}$ and drop the tilde associated to symbols on $\tilde{M}$ and identify $p=\tilde{p}$ etc. We compute for a generator $p \in \mathcal{H}_{p r}$

$$
\begin{aligned}
\mathfrak{d}_{m-1}\left(\mathfrak{d}_{m}(p)\right)= & \mathfrak{d}_{m-1}\left(\sum_{\substack{q \in \mathcal{H}_{p r} \\
\mu(q)=\mu(p)-1}} m(p, q) q\right) \\
= & \sum_{\substack{r \in \mathcal{H}_{p r} \\
\mu(r)=\mu(p)-2}} m(p, q) \cdot m(q, r) r \\
= & \sum_{\substack{q \in \mathcal{H}_{p r} \\
\mu(q)=\mu(p)-1 \\
r \in \mathcal{H}_{p r} \\
\mu(r)=\mu(p)-2}}\left(\sum_{\substack{q \in \mathcal{H}_{p r} \\
\mu(q)=\mu(p)-1}} m(p, q) \cdot m(q, r)\right) r .
\end{aligned}
$$

Thus it is enough to show for fixed $r$

$$
\sum_{\substack{q \in \mathcal{H}_{p r} \\ \mu(q)=\mu(p)-1}} m(p, q) \cdot m(q, r)=0 .
$$

If all sign products vanish we are done. If $m(p, q) \cdot m(q, r) \neq 0$ both signs $m(p, q)$ and $m(q, r)$ must be nonzero. In that case $\widehat{\mathcal{M}}(p, q)$ and $\widehat{\mathcal{M}}(q, r)$ are nonempty and by the gluing construction $\widehat{\mathcal{N}}(p, r)$ is nonempty. Theorem 30 tells us that for fixed $p$ and $r$ there are either exactly two primary cutting points $q_{u}$ and $q_{s}$ or none. We are in the first case since our $q$ is one of them. Since $m(p, q) \cdot m(q, r)=0$ for all $q \neq q_{u}, q_{s}$ the sum simplifies to

$$
m\left(p, q_{u}\right) \cdot m\left(q_{u}, r\right)+m\left(p, q_{s}\right) \cdot m\left(q_{s}, r\right)
$$

which vanishes since $m\left(p, q_{u}\right) \cdot m\left(q_{u}, r\right)=-m\left(p, q_{s}\right) \cdot m\left(q_{s}, r\right)$ by Lemma 19 .

\section{Examples}

4.1. Computation of examples. In this subsection, we discuss the aptitude and accessibility of primary Floer homology for explicit computations. We calculate two examples which arise from a slight perturbation of the integrable systems sketched in Figure 6. For simplicity assume to be in $\mathbb{R}^{2 n}$, i.e., the sets of semi-primary and primary points coincide.

If we want to compute the primary Floer homology of an explicit tangle we have to locate the primary points. Given a pair of intersecting branches of $W^{s}$ and $W^{u}$, we start at the fixed point $x$ and run simultaneously along 
(a)

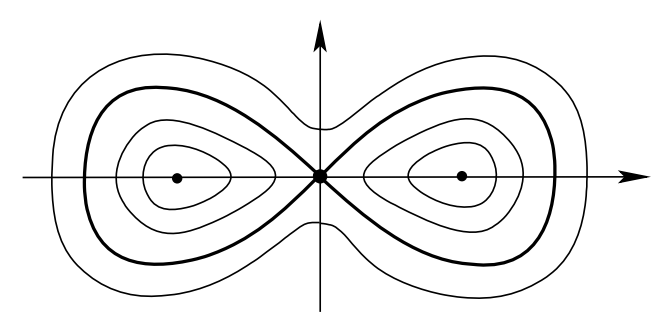

(b)

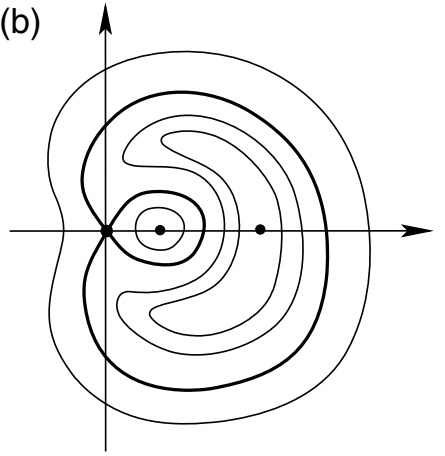

Figure 6. 'Figure eight' and 'tilted figure eight' homoclinic loops.

both branches until they intersect for the first time. This intersection point $p$ is primary. By Remark 16, all other primary points arising from this pair of branches have exactly one representant in $] p, p^{1}\left[{ }_{s} \cap\right] p, p^{1}[u$. Since all primary points are transverse there is only a finite number of primary equivalence classes and we locate their representants in $] p, p^{1}[s \cap] p, p^{1}\left[{ }_{u}\right.$ applying successively Remark 18. If we proceed in this way for all pairs of intersecting branches we obtain representatives for all primary equivalence classes.

To discover for a given $p$ all $q$ with $\mathcal{M}(p, q) \neq \emptyset$ is a little bit more tedious. Proposition 28 and Lemma 27 assure that there is only a finite number of candidates and that they are "not to far away" from $p$. Therefore it remains to check those candidates.

Thus, primary Floer homology is entirely determined by sufficiently large, fixed, compact segments of $W^{s}$ and $W^{u}$ centred around $x$. Therefore primary Floer homology can always be computed - one only needs to plot a finite part of the tangle with sufficient accuracy. Altogether, primary Floer homology provides finite information of an infinite chaotic tangle.

Using the computation of the two examples below, we will assign homology groups also to the homoclinic loops displayed in Figure 6. This will be done in Corollary 34 using the invariance property of primary Floer homology.

4.2. Figure-eight example. We compute the primary Floer homology of the schematic tangle in Figure 7. Such a tangle might arise from a figureeight homoclinic loop of an integrable system (Figure 6) by means of the Melnikov method. The hyperbolic fixed point $x$ and the elliptic fixed points $y$ and $\tilde{y}$ are printed extra bold and the Maslov grading of the primary points is given. There are eight equivalence classes $\langle p\rangle,\langle\tilde{p}\rangle,\langle q\rangle,\langle b\rangle,\langle\tilde{q}\rangle,\langle\tilde{b}\rangle,\langle r\rangle$ and $\langle\tilde{r}\rangle$ with $\mu(\langle p\rangle)=\mu(\langle\tilde{p}\rangle)=-1, \mu(\langle q\rangle)=\mu(\langle b\rangle)=\mu(\langle\tilde{q}\rangle)=\mu(\langle\tilde{b}\rangle)=-2$ 


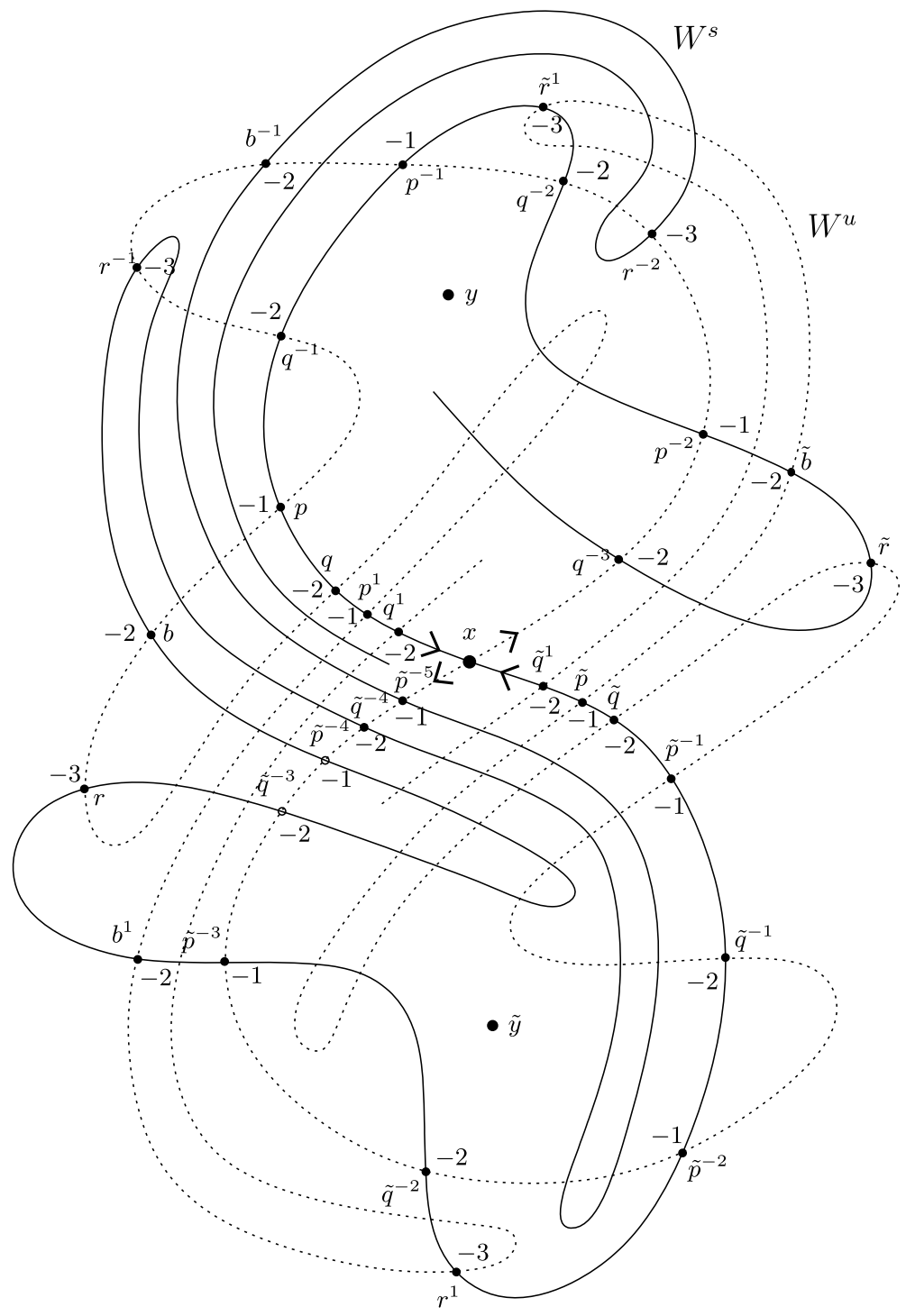

Figure 7. A 'figure eight' homoclinic tangle.

and $\mu(\langle r\rangle)=\mu(\langle\tilde{r}\rangle)=-3$. Using the $m(p, q)$-signs we obtain

$$
\begin{array}{llrl}
\partial\langle p\rangle=\langle q\rangle-\left\langle q^{-1}\right\rangle+\langle b\rangle-\left\langle\tilde{b}^{2}\right\rangle=\langle b\rangle-\langle\tilde{b}\rangle, & & \partial\langle\tilde{q}\rangle=\left\langle r^{3}\right\rangle-\langle\tilde{r}\rangle=\langle r\rangle-\langle\tilde{r}\rangle, \\
\partial\langle\tilde{p}\rangle=-\langle\tilde{q}\rangle+\left\langle\tilde{q}^{-1}\right\rangle+\langle\tilde{b}\rangle-\left\langle b^{4}\right\rangle=-\langle b\rangle+\langle\tilde{b}\rangle, & & \partial\langle\tilde{b}\rangle=-\langle\tilde{r}\rangle+\left\langle\tilde{r}^{1}\right\rangle=0, \\
\partial\langle q\rangle=-\langle r\rangle+\left\langle\tilde{r}^{3}\right\rangle=-\langle r\rangle+\langle\tilde{r}\rangle, & & \partial\langle r\rangle=0, \\
\partial\langle b\rangle=\langle r\rangle-\left\langle r^{-1}\right\rangle=0, & \partial\langle\tilde{r}\rangle=0 .
\end{array}
$$


and using the $n(p, q)$-signs with the orientation on $W^{u}$ induced by setting $x<_{u} p$ we obtain

$$
\begin{array}{llrl}
\partial\langle p\rangle=\langle q\rangle-\left\langle q^{-1}\right\rangle+\langle b\rangle-\left\langle\tilde{b}^{2}\right\rangle=\langle b\rangle-\langle\tilde{b}\rangle, & & \partial\langle\tilde{q}\rangle=\left\langle r^{3}\right\rangle+\langle\tilde{r}\rangle=\langle r\rangle+\langle\tilde{r}\rangle, \\
\partial\langle\tilde{p}\rangle=\langle\tilde{q}\rangle-\left\langle\tilde{q}^{-1}\right\rangle-\langle\tilde{b}\rangle+\left\langle b^{4}\right\rangle=\langle b\rangle-\langle\tilde{b}\rangle, & & \partial\langle\tilde{b}\rangle=\langle\tilde{r}\rangle-\left\langle\tilde{r}^{1}\right\rangle=0, \\
\partial\langle q\rangle=-\langle r\rangle-\left\langle\tilde{r}^{3}\right\rangle=-\langle r\rangle-\langle\tilde{r}\rangle=-(\langle r\rangle+\langle\tilde{r}\rangle), & & \partial\langle r\rangle=0, \\
\partial\langle b\rangle=\langle r\rangle-\left\langle r^{-1}\right\rangle=0, & & \partial\langle\tilde{r}\rangle=0 .
\end{array}
$$

The different signs lead to different boundary operators and different generators of the homologies. Nevertheless, they turn out to be isomorphic:

$$
\begin{aligned}
& H_{-1}(x, \varphi, m \text {-signs })=\mathbb{Z}(\langle p\rangle+\langle\tilde{p}\rangle) \simeq \mathbb{Z}(\langle p\rangle-\langle\tilde{p}\rangle)=H_{-1}(x, \varphi, n \text {-signs }), \\
& H_{-2}(x, \varphi, m \text {-signs })=\frac{\mathbb{Z}\langle b\rangle \oplus \mathbb{Z}\langle\tilde{b}\rangle \oplus \mathbb{Z}(\langle q\rangle+\langle\tilde{q}\rangle)}{\mathbb{Z}(\langle b\rangle-\langle\tilde{b}\rangle)}=H_{-2}(x, \varphi, n \text {-signs }), \\
& H_{-3}(x, \varphi, m \text {-signs })=\frac{\mathbb{Z}\langle r\rangle \oplus \mathbb{Z}\langle\tilde{r}\rangle}{\mathbb{Z}(\langle r\rangle-\langle\tilde{r}\rangle)} \simeq \frac{\mathbb{Z}\langle r\rangle \oplus \mathbb{Z}\langle\tilde{r}\rangle}{\mathbb{Z}(\langle r\rangle+\langle\tilde{r}\rangle)}=H_{-3}(x, \varphi, n \text {-signs }) .
\end{aligned}
$$

4.3. Tilted figure-eight example. By perturbing a tilted figure-eight homoclinic loop as in Figure 6, the homoclinic tangle of Figure 8 might arise.

There are the eight equivalence classes $\langle\tilde{p}\rangle,\langle\tilde{q}\rangle,\langle s\rangle,\langle\tilde{s}\rangle,\langle r\rangle,\langle\tilde{r}\rangle,\langle p\rangle$ and $\langle q\rangle$ with Maslov index $\mu(\langle\tilde{p}\rangle)=3, \mu(\langle\tilde{q}\rangle)=\mu(\langle s\rangle)=\mu(\langle\tilde{s}\rangle)=2, \mu(\langle r\rangle)=$ $\mu(\langle\tilde{r}\rangle)=1, \mu(\langle p\rangle)=-1$ and $\mu(\langle q\rangle)=-2$. Using the $m(p, q)$-signs, we obtain as boundary operator

$$
\begin{array}{llrl}
\partial\langle\tilde{p}\rangle=-\langle\tilde{q}\rangle+\left\langle\tilde{q}^{1}\right\rangle-\langle s\rangle+\left\langle\tilde{s}^{-3}\right\rangle=-\langle s\rangle+\langle\tilde{s}\rangle, & & \partial\langle r\rangle=0, \\
\partial\langle\tilde{q}\rangle=\left\langle r^{-1}\right\rangle-\left\langle\tilde{r}^{-3}\right\rangle=\langle r\rangle-\langle\tilde{r}\rangle, & & \partial\langle\tilde{r}\rangle=0, \\
\partial\langle s\rangle=\langle r\rangle-\left\langle r^{-1}\right\rangle=0, & & \partial\langle p\rangle=\langle q\rangle-\left\langle q^{-1}\right\rangle=0, \\
\partial\langle\tilde{s}\rangle=-\langle\tilde{r}\rangle+\left\langle\tilde{r}^{1}\right\rangle=0, & & \partial\langle q\rangle=0 .
\end{array}
$$

For the $n(p, q)$-signs, we fix an orientation of $W^{u}$ via choosing a parametrization in direction from $x$ to $p$. This leads to

$$
\begin{array}{llrl}
\partial\langle\tilde{p}\rangle & =\langle\tilde{q}\rangle-\left\langle\tilde{q}^{1}\right\rangle+\langle s\rangle-\left\langle\tilde{s}^{-3}\right\rangle=\langle s\rangle-\langle\tilde{s}\rangle, & & \partial\langle r\rangle=0, \\
\partial\langle\tilde{q}\rangle=\left\langle r^{-1}\right\rangle+\left\langle\tilde{r}^{-3}\right\rangle=\langle r\rangle+\langle\tilde{r}\rangle, & & \partial\langle\tilde{r}\rangle=0, \\
\partial\langle s\rangle=\langle r\rangle-\left\langle r^{-1}\right\rangle=0, & & \partial\langle p\rangle=\langle q\rangle-\left\langle q^{-1}\right\rangle=0, \\
\partial\langle\tilde{s}\rangle=\langle\tilde{r}\rangle-\left\langle\tilde{r}^{1}\right\rangle=0, & \partial\langle q\rangle=0 .
\end{array}
$$




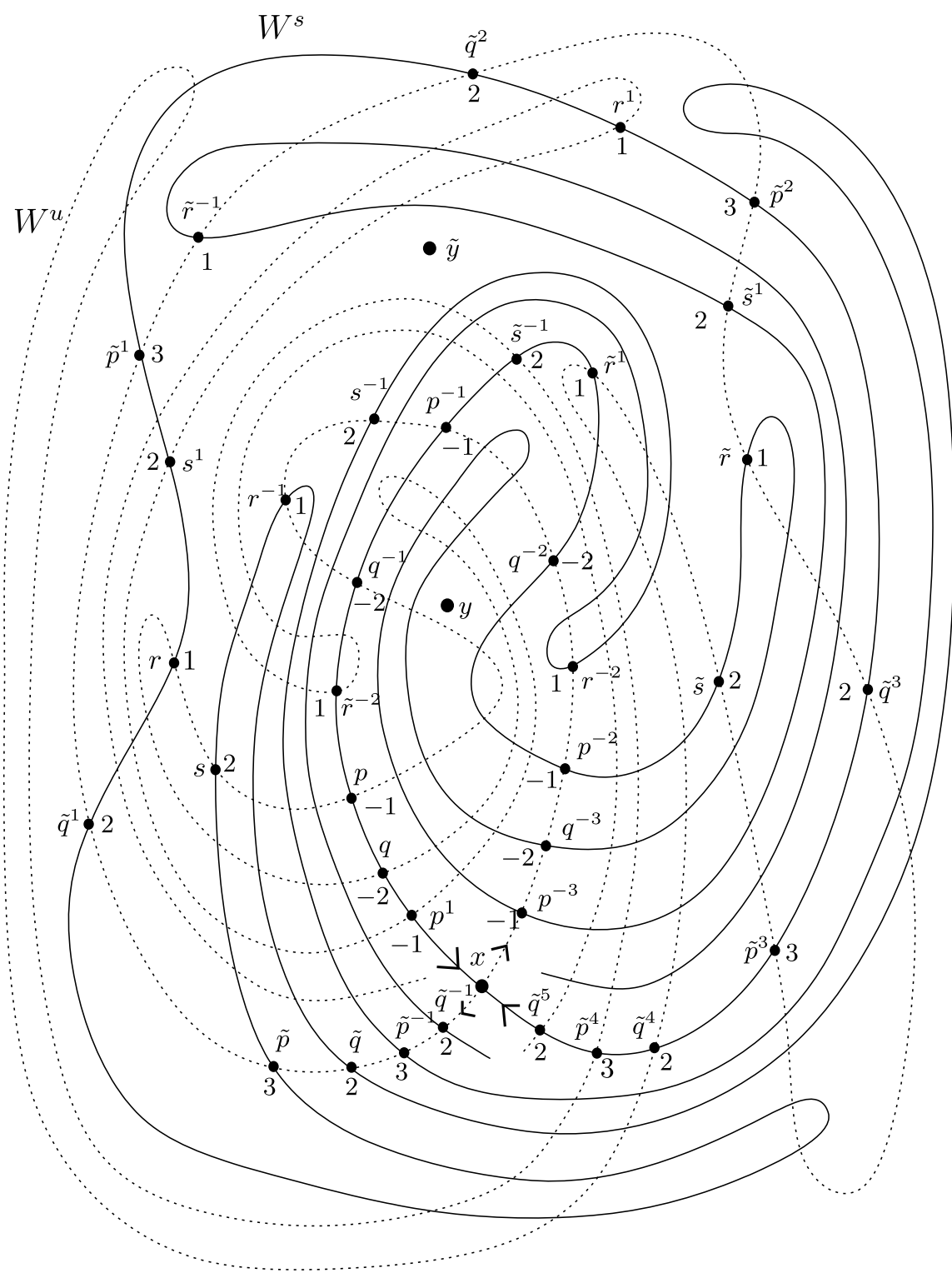

Figure 8. A 'tilted figure eight' homoclinic tangle.

As homology, we obtain $H_{l}=0$ for all $l \notin\{ \pm 1, \pm 2\}$ regardless of the chosen signs. However for the remaining four groups, we compute

$$
\begin{gathered}
H_{2}(x, \varphi, m \text {-signs })=\frac{\mathbb{Z}\langle s\rangle \oplus \mathbb{Z}\langle\tilde{s}\rangle}{\mathbb{Z}(-\langle s\rangle+\langle\tilde{s}\rangle)} \simeq \frac{\mathbb{Z}\langle s\rangle \oplus \mathbb{Z}\langle\tilde{s}\rangle}{\mathbb{Z}(\langle s\rangle-\langle\tilde{s}\rangle)}=H_{2}(x, \varphi, n \text {-signs }), \\
H_{1}(x, \varphi, m \text {-signs })=\frac{\mathbb{Z}\langle r\rangle \oplus \mathbb{Z}\langle\tilde{r}\rangle}{\mathbb{Z}(\langle r\rangle-\langle\tilde{r}\rangle)} \simeq \frac{\mathbb{Z}\langle r\rangle \oplus \mathbb{Z}\langle\tilde{r}\rangle}{\mathbb{Z}(\langle r\rangle+\langle\tilde{r}\rangle)}=H_{1}(x, \varphi, n \text {-signs }), \\
H_{-1}(x, \varphi, m \text {-signs })=\mathbb{Z}\langle p\rangle=H_{-1}(x, \varphi, n \text {-signs }) \\
H_{-2}(x, \varphi, m \text {-signs })=\mathbb{Z}\langle q\rangle=H_{-2}(x, \varphi, n \text {-signs }) .
\end{gathered}
$$




\section{Invariance}

In classical Lagrangian Floer theory, invariance of the homology under Hamiltonian perturbations of the underlying Lagrangians is an important feature. Thus one can choose a particular nice Lagrangian within the Hamiltonian isotopy class for the computation of the homology.

Our situation differs strongly from the classical one. Whereas in the classical situation a Hamiltonian diffeomorphism $f$ is applied directly to a Lagrangian $L$ changing it to $f(L)$, the change here occurs indirectly. We are going to perturb the underlying symplectomorphism $\varphi$ which results in changing both the stable and unstable manifolds. Our invariance proof is inspired by Floer's original proof in [F13] which uses explicit chain homotopies. The more modern ansatz via homotopy of homotopies is unfortunately not applicable since it is not compatible with the bifurcation nature of primary points. We will use the invariance to assign homology groups to the homoclinic loops in Figure 6.

Primary points are printed extra bold in figures. In order to obtain smaller sketches, we sometimes draw the hyperbolic fixed point $x$ 'splitted' into two copies. In this section, $(M, \omega)$ is a closed symplectic two-dimensional manifold with genus $g \geq 1$. The group of smooth diffeomorphisms $\operatorname{Diff}(M)$ is endowed with the Whitney topology (which coincides on compact manifolds with the $C^{r}$-topology) and $\operatorname{Diff}_{\omega}(M) \subset \operatorname{Diff}(M)$ is the group of symplectomorphisms.

5.1. Main results. Let $\varphi \in \operatorname{Diff}^{k}(M)$ with $k \geq 1$ and $x \in \operatorname{Fix}(\varphi)$ hyperbolic and $\psi \in \operatorname{Diff}^{k}(M)$ sufficiently $C^{k}$-near to $\varphi$. Then it is well known that $\psi$ has a hyperbolic fixed point $y$ near $x . W^{i}(y, \psi)$ is $C^{k}$-near $W^{i}(x, \varphi)$ for $i \in\{u, s\}$, at least on compact neighbourhoods of $y$ and $x$ in $W^{i}(y, \psi)$ and $W^{i}(x, \varphi) . y$ is called the continuation of $x$ and the signs of the corresponding eigenvalues coincide.

Let $\varphi, \psi \in \operatorname{Diff}_{\omega}(M)$ and $x \in \operatorname{Fix}(\varphi)$ and $y \in \operatorname{Fix}(\psi)$ both hyperbolic. An isotopy (between $(x, \varphi)$ and $(y, \psi)$ ) is a smooth path $\Phi:[0,1] \rightarrow \operatorname{Diff}_{\omega}(M)$, $\tau \mapsto \Phi(\tau)=: \Phi_{\tau}$ with $\Phi_{0}=\varphi, \Phi_{1}=\psi, x_{0}=x$ and $x_{1}=y$ and $x_{\tau} \in \operatorname{Fix}\left(\Phi_{\tau}\right)$ as continuation between $x$ and $y$ for all $\tau \in[0,1]$. Attaching $\tau$ to a symbol associates it to $\left(x_{\tau}, \Phi_{\tau}\right)$, i.e., $\mathcal{H}_{p r}^{\tau}$ denotes the set of primary points of $\left(x_{\tau}, \Phi_{\tau}\right)$ etc. $(x, \varphi)$ is called contractibly strongly intersecting (csi) if $W^{u}$ and $W^{s}$ are strongly intersecting and if each pair of branches has contractible homoclinic points. An isotopy $\Phi$ is csi if $\left(x_{\tau}, \Phi_{\tau}\right)$ is csi for all $\tau \in[0,1]$.

Theorem 31 (Invariance). Let $(M, \omega)$ be a closed symplectic two-dimensional manifold with genus $g \geq 1$. Let $\varphi, \psi \in \operatorname{Diff}_{\omega}(M)$ with hyperbolic fixed points $x \in \operatorname{Fix}(\varphi)$ and $y \in \operatorname{Fix}(\psi)$. Let $(x, \varphi)$ and $(y, \psi)$ be

csi and let all primary points of $\varphi$ and $\psi$ be transverse. Assume there is a 
(a)

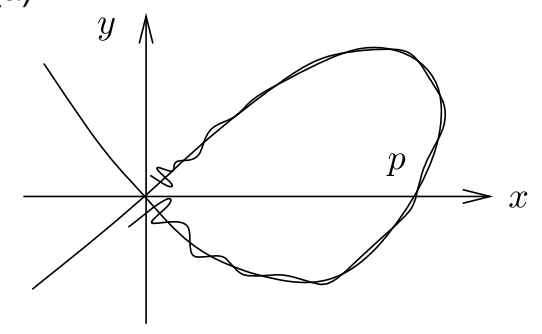

(b)

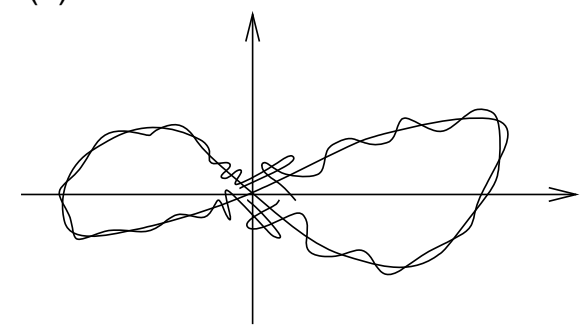

Figure 9. Homoclinic tangle of the quadratic map (a) and the cubic map (b).

csi isotopy $\Phi$ from $(x, \varphi)$ to $(y, \psi)$. Then

$$
H_{*}(x, \varphi) \simeq H_{*}(y, \psi) .
$$

We will prove Theorem 31 in the following subsections. The proof carries over to compactly supported symplectomorphisms on $\mathbb{R}^{2}$. 'Csi' and 'compactly supported' are crucial:

Remark 32. There are $\varphi, \psi \in \operatorname{Diff}_{d x \wedge d y}\left(\mathbb{R}^{2}\right)$ with hyperbolic fixed points $x \in \operatorname{Fix}(\varphi)$ and $y \in \operatorname{Fix}(\psi)$ which can be joint by a symplectic isotopy and which have

(1) different number of pairs of intersecting branches,

(2) $H_{*}(x, \varphi) \neq H_{*}(y, \psi)$.

Proof. For small $\varepsilon>0$, consider the path $\Phi^{\varepsilon}:[0,1] \rightarrow \operatorname{Diff}_{d x \wedge d y}\left(\mathbb{R}^{2}\right)$ given by

$$
\Phi_{\tau}^{\varepsilon}(x, y):=\left(x+y+\varepsilon f_{\tau}(x), y+\varepsilon f_{\tau}(x)\right)
$$

with $f_{\tau}(x):=-\tau x^{3}-(1-\tau) x^{2}+x$. We have $\Phi_{\tau}^{\varepsilon}(0,0)=(0,0)$ with $D \Phi_{\tau}^{\varepsilon}(0,0)=\left(\begin{array}{cc}1+\varepsilon & 1 \\ \varepsilon & 1\end{array}\right)$ as hyperbolic fixed point. Now set $\varphi:=\Phi_{0}^{\varepsilon}$ and $\psi:=\Phi_{1}^{\varepsilon} . \varphi$ is the volume preserving Hénon map and its homoclinic tangle is sketched in Figure 9 (a): $\varphi$ has one pair of intersecting branches. The tangle of $\psi$ is sketched in Figure $9(\mathrm{~b})$ and has four pairs of intersecting branches. We compute $H_{2}((0,0), \varphi) \simeq \mathbb{Z}, H_{1}((0,0), \varphi) \simeq \mathbb{Z}$ and $H_{n}((0,0), \varphi)=0$ otherwise. However, $\psi$ has $H_{3}((0,0), \psi) \neq 0$, thus $H_{*}((0,0), \varphi) \neq H_{*}((0,0), \psi)$.

As an application, we obtain the following statement.

Corollary 33 (existence and bifurcation criterion). Assume the conditions of Theorem 31 for $(M, \omega),(x, \varphi)$ and $(y, \psi)$, but $H_{*}(x, \varphi) \neq H_{*}(y, \psi)$. Then $(x, \varphi)$ and $(y, \psi)$ cannot be joint by a csi isotopy. Thus, if there is a path $\left(\Phi_{\tau}\right)_{\tau \in[0,1]} \in \operatorname{Diff}_{\omega}(M)$ between $\varphi$ and $\psi$ then

(1) either $\Phi$ is no isotopy, i.e., there is $\tau_{0} \in[0,1]$ where $x_{\tau_{0}}$ vanishes or undergoes a bifurcation, 
(2) or, if $\Phi$ is an isotopy, there has to be a pair of branches and some $\tau_{0} \in[0,1]$ where all contractible homoclinic points vanish, i.e., there are homoclinic bifurcations.

Now apply the invariance property to assign homology groups to homoclinic loops of autonomous Hamiltonian systems. Let $H: \mathbb{R}^{2} \rightarrow \mathbb{R}$ be a compactly supported, autonomous smooth Hamiltonian function and $X$ its Hamiltonian vector field. Assume $X$ to have locally a phase portrait like Figure 6 (a) or (b). Let $\varepsilon>0$ be small and let $Y$ be a smooth nonautonomous vector field with support in the support of $H$.

By Melnikov's method [Me, GH], the time-one map $\varphi_{\varepsilon}$ of the nonautonomous system $\dot{z}(t)=X(z(t))+\varepsilon Y(t, z(t))$ now has a homoclinic tangle instead of a homoclinic loop. The Melnikov function measures the existence and "width" of the arising homoclinic tangle (for higher iterates see RomKedar $[\mathbf{R K 1 , R K 2}])$. Therefore one knows quite well how the tangle behaves for $\varepsilon \rightarrow 0$.

In case of Figure 6 (a), the tangle looks roughly like the one of Figure 7. In case of Figure 6 (b), the tangle resembles somewhat Figure 8. The isotopy $\varepsilon \mapsto \varphi_{\varepsilon}$ satisfies the requirements of Theorem 31 for small $\varepsilon>0$. The natural parametrization of the homoclinic loops induces the signs of the Maslov indices: If the loop winds (counter)clockwise, the arising branches of the unstable and stable manifold have primary points with positive (negative) Maslov index. We summarize this as follows.

Corollary 34. Figure-eight homoclinic loops are characterized by

$$
H_{\sigma \cdot 1}=\mathbb{Z}, \quad H_{\sigma \cdot 2}=\mathbb{Z} \oplus \mathbb{Z}, \quad H_{\sigma \cdot 3}=\mathbb{Z}
$$

where $\sigma=1$ for clockwise and $\sigma=-1$ for counterclockwise parametrization. In case of the tilted figure-eight homoclinic loop, both cases lead, due to symmetry, to

$$
H_{-2}=H_{-1}=H_{1}=H_{2}=\mathbb{Z}, \quad H_{ \pm 3}=0 .
$$

In Theorem 31, we impose the transversality condition only on the primary points of $(x, \varphi)$ and $(y, \psi)$. This is convenient for applications since it can be checked easily using Remark 16 and Remark 18. However, our proof strategy requires perturbations of $\Phi$. Thus we have to show that slight perturbations of the start and endpoint preserve the associated primary Floer homologies.

Proposition 35. Let $(M, \omega)$ be a closed, two-dimensional symplectic manifold with genus $g \geq 1$. Let $\varphi \in \operatorname{Diff}_{\omega}(M)$ and $x \in \operatorname{Fix}(\varphi)$ hyperbolic. Let $(x, \varphi)$ be csi and all primary points transverse. Then, for all $\hat{\varphi} \in \operatorname{Diff}_{\omega}(M)$ sufficiently close to $\varphi$, holds

$$
H_{*}(x, \varphi)=H_{*}(\hat{x}, \hat{\varphi}) .
$$

where $\hat{x} \in \operatorname{Fix}(\hat{\varphi})$ is the continuation of $x$. 
The proof of Proposition 35 is postponed to Paragraph 5.6.

Remark 36. (1) Whereas, in the two-dimensional setting, primary Floer homology can be defined also for nonsymplectic diffeomorphisms, invariance only is natural within the class of symplectomorphisms.

(2) We conjecture that Hamiltonian diffeomorphisms are naturally csi.

(3) In contrast to classical Lagrangian Floer theory, invariance of primary Floer homology relies on the nontrivial result of (generical) existence of intersection points of the Lagrangians.

5.2. Outline of the proof of Theorem 31. In order to prove Theorem 31, we have to deal with bifurcations of homoclinic points. We denote the bifurcation parameter by $\tau$ with tangency at $\tau_{0}$. Generically, a homoclinic tangency is simple and the picture looks (after a suitable symplectic coordinate change) locally like the graph of $f+C\left(\tau-\tau_{0}\right)$ intersecting the $x$-axis where $f$ is a quadratic, homogenous and nondegenerate function and $C>0$.

Passing from $\tau<\tau_{0}$ to $\tau>\tau_{0}$ (or vice versa), we briefly call a move and omit the bifurcation parameter $\tau$. By abuse of notation, we speak of an $(r, s)$-move if the arising resp. vanishing two points are called $r$ and $s$. In fact,

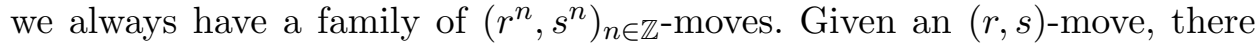
is always an embedded di-gon between $r$ and $s$ since $] r, s\left[{ }_{u} \cap\right] r, s\left[{ }_{s}=\emptyset\right.$. If $r$ and $s$ are primary then they are adjacent to each other. Moreover, $x \notin[r, s]_{u} \cup[r, s]_{s}$ and therefore $r$ and $s$ always lie on the same branches.

Proof of Theorem 31. First, we perturb the isotopy $\Phi$ in order to obtain the above mentioned generic bifurcations. This is possible since csi is an open property in $\operatorname{Diff}_{\omega}(M)$. Since the set of all $\tau \in[0,1]$, for which all primary points are nontransverse, is discrete we can perturb the isotopy once again slightly, in order to obtain for all $\tau \in[0,1]$ a transverse primary point within each pair of intersection branches.

Recall the properties of primary points from Remark 16 and Remark 18. Given a primary $p$, we call $\left[p, p^{1}\right]_{u} \cup\left[p, p^{1}\right]_{s}$ together with the positions of $\left[p, p^{1}\right]_{u} \cap\left[p, p^{1}\right]_{s}$ and the immersions (embeddings on $\tilde{M}$ ) between adjacent points the frame induced by $p$. Every primary equivalence class associated to that pair of branches and different from $p$ has exactly one representative in the frame induced by $p$. As long as $p$ persist as primary point its frame allows to observe all primary bifurcations. Now we cover $[0,1]$ by a finite number of overlapping intervals associated to persisting primary points.

Primary points can only arise resp. vanish at certain distinguished parts of the compact frame segments $\left[p, p^{1}\right]_{u}$ and $\left[p, p^{1}\right]_{s}$, see Remark 18 and Figure 13. Thus there are only finitely many $\tau \in[0,1]$, where primary points can arise resp. vanish. As Proposition 39 and Lemma 52 will show, bifurcations of secondary points either do not affect the chain complex or are 


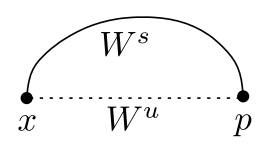

(i)

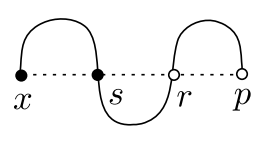

(ii)

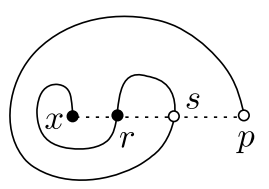

(iii)

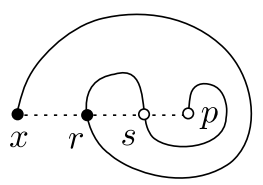

(iv)

Figure 10. Causes for a primary-secondary flip.

coupled to certain primary bifurcations. Since primary Floer theory lives within compact segments centred around the fixed point, we can model the relevant bifurcations of the isotopy as a sequence of moves as in knot theory.

Now let us discuss the moves more detailed. W.l.o.g. we will assume from now on that, in case of a bifurcation in $p$ at time $\tau_{0}$, the tangency $p$ unfolds into two points for $\tau>\tau_{0}$ and vanishes for $\tau<\tau_{0}$. This we briefly call after resp. before the bifurcation or move. We call a point involved in a move if it is either the homoclinic tangency at time $\tau_{0}$ or one of the arising transverse homoclinic points. Persistent transverse primary points $p$ and $q$ are called combinatorically affected by a move if the value of $m(p, q)$ is changed by the move. By abuse of notation, we call in this case also the elements of $\mathcal{M}(p, q)$ affected by the move.

There are two possibilities to generate (analogously destroy) a primary point $p$ by a move:

(a) $p$ arises as intersection point.

(b) $p$ was secondary and becomes primary. This phenomenon we call a primary-secondary flip, briefly a flip.

In the latter case, the point does not necessarily need to be involved in the move itself, cf. Figure 13. Primary points cannot switch to nontrivial homotopy classes or vice versa due to ' $\cdots \cap \mathcal{H}_{[x]}$ ' in the definition of 'primary'.

Since there are always two points involved in a bifurcation the following types of moves are possible:

(a) If both arising points are primary the move is called primary.

(b) If one of the arising points is primary and the other one secondary the move is called mixed.

(c) If both arising points are secondary the move is called secondary.

We note

Lemma 37. Let $p$ be not involved itself in a given move, but let $p$ undergo a primary-secondary flip. Then the move is a mixed one.

Proof of Lemma 37. Consider the lifted tangle. The situation is sketched in Figure 10. In (i), $p$ is primary before the move. In (ii - iv), the possible types of moves are listed which turn $p$ secondary - all of them are mixed. 
Having Lemma 37 and Figure 10 in mind, we conclude the following changes of the set of primary points under the different types of moves.

Corollary 38. (1) A primary move generates two primary points and does not flip any.

(2) A mixed move generates one primary point, but flips a certain number of primary points secondary.

(3) A secondary move neither generates primary points nor can flip some of them secondary, i.e., the set of primary points stays untouched.

Corollary 38 characterizes how the different types of moves affect the generator set of the chain groups. We will inquire about the potential changes of the boundary operator in the next subsections. It will be proven in Proposition 39, Theorem 47, Theorem 48 and Theorem 50 that all three kinds of moves leave the homology invariant which proves Theorem 31 .

5.3. Invariance under secondary moves. For simplicity, we work with the lifted homoclinic tangle on the universal cover. According to Corollary 38 , the generator set of the chain complex stays unchanged under secondary moves and we will show now that this is also true for the boundary operator.

Proposition 39. Secondary moves do not affect embeddings between primary points.

Proof. We argue by contradiction: Let $u$ be an embedding between primary points $p$ and $q$ with $\mu(p, q)=1$. Consider an $(r, s)$-move such that $\{r, s\}=$ ]$p, q[u \cap] p, q[s$. We show: If $r$ and $s$ are secondary then the $(r, s)$-move already flipped either $p$ or $q$ secondary before $r$ and $s$ could arise. The proof is tedious, but elementary. We just have to check for the embeddings of Figure 4 all combinatorial possibilities of $(r, s)$-moves affecting the boundary $[p, q]_{u} \cup[p, q]_{s}$ such that $\left.\{r, s\}=\right] p, q\left[{ }_{u} \cap\right] p, q[s$.

We only prove the assertion exemplarily in the case of Figure 4 (i) with $\mu(p, q)=(-1,2)$ which is resketched in Figure 11 (i). The strategy and result for the other cases in Figure 4 are the same.

Consider Figure 11 (i) and the boundary $[p, q]_{u} \cup[p, q]_{s}$ of the embedding between $p$ and $q.] p, q[u \backslash\{x\}$ consists of the two connected components $] p, x[u$ and $] q, x\left[{ }_{u}\right.$. Since $r$ and $s$ always lie in the same branch we have to distinguish the cases $r, s \in] p, x\left[_{u}\right.$ (see Figure 11 (ii), (vi), (vii)) and $\left.r, s \in\right] q, x\left[_{u}\right.$ (see Figure 11 (iii), (iv), (v)). Moreover, we have to distinguish if $p$ is connected within $W^{s}$ first to $s$ (see Figure 11 (ii), (iii)) or to $r$ (see Figure 11 (iv)-(vii)). The cases (iv) and (v) on the one hand and (vi) and (vii) on the other hand are basically the same.

We deduce that (ii) is a primary move and that (iii), (iv) and (v) are mixed ones. In (vi) and (vii) the points $r$ and $s$ are both secondary. However, before the move, starting in the situation of (i), generates the intersection points $r$ 


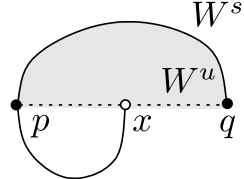

(i)

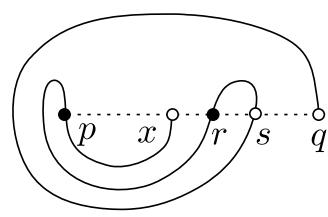

(v)

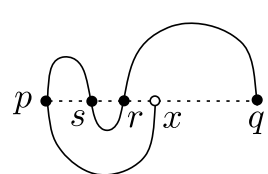

(ii)

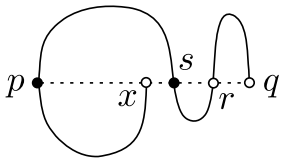

(iii)

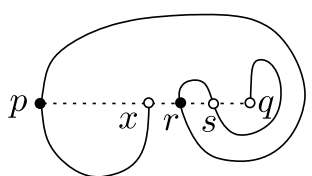

(iv)

Figure 11. The effect of moves on an embedding between primary points $p$ and $q$.

(i)

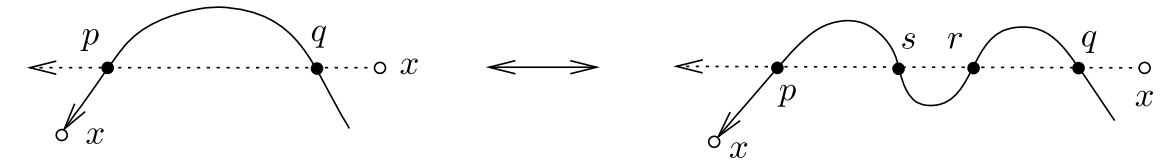

(ii)
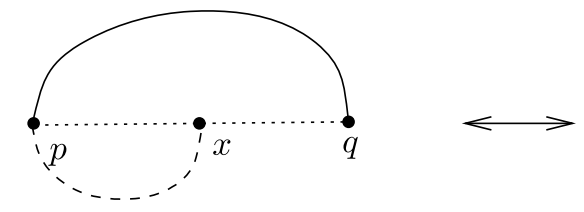

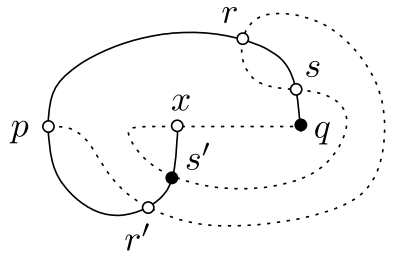

(vii)

(vi)

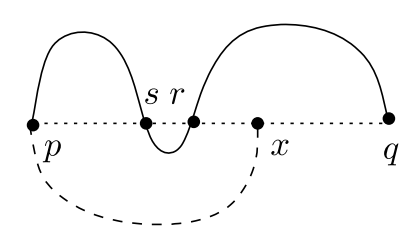

Figure 12. The primary $(r, s)$-move.

and $s$ in (vi) and (vii) it has to pass through $] p, x[s$ generating the intersection points $r^{\prime}$ and $s^{\prime}$, which yields a mixed $\left(r^{\prime}, s^{\prime}\right)$-move flipping $p$ secondary.

Proposition 39 and Corollary 38 imply the invariance of primary Floer homology under secondary moves. Moreover, we note that, according to the proof of Proposition 39, a mixed move affecting an embedding between two primary points always flips one of them secondary.

5.4. Invariance under primary moves. Now we prove the invariance of primary Floer homology under primary moves. The proof generalizes ideas of Floer $[\mathbf{F l 3}]$ and de Silva $[\mathbf{d S}]$.

In Figure 12, the two possibilities for primary moves are sketched which are deduced from Figure 4 (up to symmetries). 
Given $u \in \mathcal{M}(p, q)$, consider a primary $(r, s)$-move such that for some $m \in \mathbb{Z}$ the family element $\left(r^{m}, s^{m}\right)$ affects $u$, i.e., $\left.\left[r^{m}, s^{m}\right]_{i} \subset\right] p, q[i$ for $i \in\{u, s\}$. Then there is no $n \in \mathbb{Z}^{\neq m}$ such that $\left(r^{n}, s^{n}\right)$ affects $u$ : Let the symplectomorphism be $W$-orientation preserving and w.l.o.g. $m=0$. Since $x \notin[p, q]_{u} \cap[p, q]_{s}$ at least one of the points $p, q$ lies in the same branch as $r$ and $s$ and w.l.o.g. let it be $p$. If there would be an $n \neq 0$ with $\left.\left[r^{n}, s^{n}\right]_{i} \subset\right] p, q[i$ then there is an iterate $p^{k}$ with $\left.p^{k} \in\left[r, r^{n}\right]_{u} \cap\left[r, r^{n}\right]_{s} \subset\right] p, q\left[{ }_{u} \cap\right] p, q\left[_{s}\right.$. However, then already $\left.p^{k} \in\right] p, q\left[{ }_{u} \cap\right] p, q[s$ before the primary $(r, s)$-move took place. Thus $u$ is no embedding. For a $W$-orientation reversing symplectomorphism consider its square.

We denote by $\langle\cdot, \cdot\rangle: \mathcal{H}_{p r} \times \mathcal{H}_{p r} \rightarrow\{0,1\}$ the Kronecker symbol and extend it to the chain complex by linearity.

For an isotopy $\Phi$ which has a primary tangency at $\tau_{0}$ and which displays a primary $(r, s)$-move for $\tau \in\left[\tau_{0}-\varepsilon, \tau_{0}+\varepsilon\right]$ we abbreviate $\mathcal{H}_{p r}:=\mathcal{H}_{p r}\left(\Phi_{\tau_{0}-\varepsilon}, x_{\tau_{0}-\varepsilon}\right)$ and identify $\mathcal{H}_{p r}^{\prime}:=\mathcal{H}_{p r}\left(\Phi_{\tau_{0}+\varepsilon}, x_{\tau_{0}+\varepsilon}\right)=\mathcal{H}_{p r} \cup$ $\left\{r^{n}, s^{n} \mid n \in \mathbb{Z}\right\}$. Moreover set $\left(\mathfrak{C}_{*}, \mathfrak{d}\right):=\left(\mathfrak{C}_{*}\left(x_{\tau_{0}-\varepsilon}, \Phi_{\tau_{0}-\varepsilon}\right), \mathfrak{d}_{x_{\tau_{0}-\varepsilon}, \Phi_{\tau_{0}-\varepsilon}}\right)$ and $\left(\mathfrak{C}_{*}^{\prime}, \mathfrak{d}^{\prime}\right):=\left(\mathfrak{C}_{*}\left(x_{\tau_{0}+\varepsilon}, \Phi_{\tau_{0}+\varepsilon}\right), \mathfrak{d}_{\tau_{\tau_{0}+\varepsilon}, \Phi_{\tau_{0}+\varepsilon}}\right)$. Mark signs after the move by a prime, i.e., $m^{\prime}(\cdot, \cdot)$. Given a primary $(r, s)$-move, we define the projection

$$
\pi: \mathfrak{C}_{*}^{\prime} \rightarrow \mathfrak{C}_{*}, \quad \pi(p)=p-\sum_{n \in \mathbb{Z}}\left\langle p, r^{n}\right\rangle r^{n}-\left\langle p, s^{n}\right\rangle s^{n} .
$$

The inclusion $\mathcal{H}_{p r} \hookrightarrow \mathcal{H}_{p r}^{\prime}$ induces the homomorphism $i: \mathfrak{C}_{*} \rightarrow \mathfrak{C}_{*}^{\prime} . \pi$ and $i$ commute with the $\mathbb{Z}$-action on the chain complexes. W.l.o.g. assume for the remaining subsection that for a primary $(r, s)$-move holds $\mu(r, s)=1$ as sketched in Figure 12.

Proposition 40. For all primary $p, q \in \mathcal{H}_{p r}$ and all primary $(r, s)$-moves holds

$$
m(p, q)=m^{\prime}(\mathrm{i}(p), \mathrm{i}(q))-\sum_{n \in \mathbb{Z}} m^{\prime}\left(\mathrm{i}(p), s^{n}\right) m^{\prime}\left(r^{n}, s^{n}\right) m^{\prime}\left(r^{n}, \mathrm{i}(q)\right) .
$$

Proof. We know that the primary $(r, s)$-move changes $\mathcal{H}_{p r}$ to $\mathcal{H}_{p r}^{\prime}=\mathcal{H}_{p r} \cup$ $\left\{r^{n}, s^{n} \mid n \in \mathbb{Z}\right\}$. Figure 12 sketches the possible geometric positions of $p, q$, $r, s$. Recall that for primary points $p$ and $q$ an embedding $u \in \mathcal{M}(p, q)$ is combinatorically affected by the primary $(r, s)$-move if and only if there is exactly one $n \in \mathbb{Z}$ such that $] p, q\left[{ }_{u} \cap\right] p, q\left[{ }_{s}=\left\{r^{n}, s^{n}\right\}\right.$ after the move.

If the embedding is combinatorically affected by $r^{n}$ and $s^{n}$ then it corresponds under the move to three embeddings between $r^{n}$ and $q, r^{n}$ and $s^{n}$ and $p$ and $s^{n}$. Using some gluing construction within a small neighbourhood $U$ containing the move, we obtain $\widehat{\mathcal{M}}(p, q) \simeq \widehat{\mathcal{M}}\left(r^{n}, q\right) \times \widehat{\mathcal{M}}\left(r^{n}, s\right) \times \widehat{\mathcal{M}}\left(p, s^{n}\right)$. Counting with orientation, we find $m(p, q)=m^{\prime}\left(\mathrm{i}(p), s^{n}\right)=m^{\prime}\left(r^{n}, \mathrm{i}(q)\right)=$ $-m^{\prime}\left(r^{n}, s^{n}\right)$ and thus $m(p, q)=-m^{\prime}\left(\mathrm{i}(p), s^{n}\right) m^{\prime}\left(r^{n}, s^{n}\right) m^{\prime}\left(r^{n}, \mathrm{i}(q)\right)$. For $k \in$ $\mathbb{Z}^{\neq n}$ the embedding $u \in \mathcal{M}(p, q)$ stays unchanged and $m^{\prime}\left(i(p), s^{k}\right) m^{\prime}\left(r^{k}, s^{k}\right) m^{\prime}$ $\left(r^{k}, i(q)\right)=0$. 
If $u$ is not combinatorically affected by the move then either $\widehat{\mathcal{M}}\left(p, s^{l}\right)=\emptyset$ or $\widehat{\mathcal{M}}\left(r^{l}, q\right)=\emptyset$ for all $l \in \mathbb{Z}$. In this case we have $-m^{\prime}\left(\mathrm{i}(p), s^{l}\right) m^{\prime}\left(r^{l}, s^{l}\right)$ $m^{\prime}\left(r^{l}, \mathrm{i}(q)\right)=0$ and $m(p, q)=m^{\prime}(\mathrm{i}(p), \mathrm{i}(q))$, thus altogether $m(p, q)=$ $m^{\prime}(\mathrm{i}(p), \mathrm{i}(q))-\sum_{n \in \mathbb{Z}} m^{\prime}\left(\mathrm{i}(p), s^{n}\right) m^{\prime}\left(r^{n}, s^{n}\right) m^{\prime}\left(r^{n}, \mathrm{i}(q)\right)$.

Now we express the boundary operator $\mathfrak{d}$ in terms of $\mathfrak{d}^{\prime}$.

\section{Lemma 41.}

$$
\begin{aligned}
& \mathfrak{d} p=\pi\left(\mathfrak{d}^{\prime} \mathrm{i}(p)-\sum_{n \in \mathbb{Z}} m^{\prime}\left(\mathrm{i}(p), s^{n}\right) m^{\prime}\left(r^{n}, s^{n}\right) \mathfrak{d}^{\prime} r^{n}\right) \quad \text { for } \mu(\mathrm{i}(p), r)=0, \\
& \mathfrak{d} p=\pi\left(\mathfrak{d}^{\prime} \mathrm{i}(p)\right) \quad \text { otherwise. }
\end{aligned}
$$

Proof. We compute formally

$$
\begin{aligned}
\mathfrak{d}^{\prime} i(p) & =\sum_{\substack{\mu(i(p), \tilde{q})=1 \\
\tilde{q} \notin\left\{r^{n}, s^{n} \mid n \in \mathbb{Z}\right\}}} m^{\prime}(\mathrm{i}(p), \tilde{q}) \tilde{q}+\sum_{n \in \mathbb{Z}} m^{\prime}\left(\mathrm{i}(p), r^{n}\right) r^{n}+\sum_{n \in \mathbb{Z}} m^{\prime}\left(\mathrm{i}(p), s^{n}\right) s^{n}, \\
\mathfrak{d}^{\prime} r^{m} & =\sum_{\substack{\mu(r m, \tilde{q})=1 \\
\tilde{q} \notin\left\{s^{n} \mid n \in \mathbb{Z}\right\}}} m^{\prime}(\mathrm{i}(p), \tilde{q}) \tilde{q}+\sum_{n \in \mathbb{Z}} m^{\prime}\left(r^{m}, s^{n}\right) s^{n}
\end{aligned}
$$

and, making use of the Kronecker symbol via $\langle\partial p, q\rangle=m(p, q)$ etc., we rewrite Proposition 40 as

$$
\langle\mathfrak{d} p, q\rangle=\left\langle\mathfrak{d}^{\prime} \mathrm{i}(p)-\sum_{n \in \mathbb{Z}} m^{\prime}\left(\mathrm{i}(p), s^{n}\right) m^{\prime}\left(r^{n}, s^{n}\right) \mathfrak{d}^{\prime} r^{n}, \mathrm{i}(q)\right\rangle .
$$

Applying $\pi$ to $\mathfrak{d}^{\prime} i(p)$ and $\mathfrak{d}^{\prime} r^{m}$ kills all $r^{n}$ - and $s^{n}$-terms. We end up exactly with those terms which occur (maybe multiplied by $m^{\prime}\left(i(p), s^{n}\right) m^{\prime}\left(r^{n}, s^{n}\right)$ ) in (42). So we obtain $\mathfrak{d} p=\pi\left(\mathfrak{d}^{\prime} i(p)-\sum_{n \in \mathbb{Z}} m^{\prime}\left(i(p), s^{n}\right) m^{\prime}\left(r^{n}, s^{n}\right) \mathfrak{d}^{\prime} r^{n}\right)$ for $\mu(i(p), r)=0$ and $\mathfrak{d} p=\pi\left(\mathfrak{d}^{\prime} i(p)\right)$ otherwise.

Now note the following technical statement:

Lemma 43. Consider a primary $(r, s)$-move. Then for $k, l \in \mathbb{Z}$ holds $m^{\prime}\left(r^{k}, s^{l}\right)=0$ for $k \neq l$.

Proof. For fixed $m$, the points $r^{m}$ and $s^{m}$ are adjacent, but not $r^{m}$ and $s^{m-1}$ and $s^{m}$ and $r^{m+1}$ : otherwise $\langle r\rangle$ and $\langle l\rangle$ would be the only primary equivalence classes of their pair of intersecting branches implying nonintersecting branches before the move in contradiction to the assumption on the isotopy. From Remark 18 and Remark 18 we deduce $] r^{m}, s^{n}\left[{ }_{u} \cap\right] r^{m}, s^{n}[s \neq \emptyset$ for $|m-n| \geq 1$ and thus $\mathcal{M}\left(r^{m}, s^{n}\right)=\emptyset$ and $m^{\prime}\left(r^{m}, s^{n}\right)=0$.

For the following proofs, keep in mind that $m(p, q) m(p, q) \in\{0,1\}$. 
Lemma 44. We define on the generators

$$
\begin{aligned}
& f:\left(\mathfrak{C}_{*}^{\prime}, \mathfrak{d}^{\prime}\right) \rightarrow\left(\mathfrak{C}_{*}, \mathfrak{d}\right), \quad f(p):=\pi\left(p-\sum_{n \in \mathbb{Z}} m^{\prime}\left(r^{n}, s^{n}\right)\left\langle p, s^{n}\right\rangle \mathfrak{d}^{\prime} r^{n}\right), \\
& g:\left(\mathfrak{C}_{*}, \mathfrak{d}\right) \rightarrow\left(\mathfrak{C}_{*}^{\prime}, \mathfrak{d}^{\prime}\right), \quad g(p):=\mathrm{i}(p)-\sum_{n \in \mathbb{Z}} m^{\prime}\left(r^{n}, s^{n}\right) m^{\prime}\left(\mathrm{i}(p), s^{n}\right) r^{n}
\end{aligned}
$$

and extend them by linearity. Then $f$ and $g$ are $\mathbb{Z}$-equivariant chain maps.

Proof. For $m \in \mathbb{Z}$ we compute

$$
f\left(r^{m}\right)=0, \quad f\left(s^{m}\right)=-m^{\prime}\left(r^{m}, s^{m}\right) \pi \mathfrak{d}^{\prime} r^{m}, \quad f(p)=\pi(p) \quad \text { for } p \neq r^{m}, s^{m} .
$$

Recall $\mu\left(r^{m}, s^{m}\right)=1$ and $\widehat{\mathcal{M}}\left(r^{m}, s^{m}\right) \neq \emptyset$ such that $m^{\prime}\left(r^{m}, s^{m}\right)= \pm 1$ and keep the equations

$$
\begin{aligned}
& \mathfrak{d} p=\pi\left(\mathfrak{d}^{\prime} \mathrm{i}(p)-\sum_{n \in \mathbb{Z}} m^{\prime}\left(p, s^{n}\right) m^{\prime}\left(r^{n}, s^{n}\right) \mathfrak{d}^{\prime} r^{n}\right) \quad \text { for } \mu(\mathrm{i}(p), r)=0, \\
& \mathfrak{d} p=\pi\left(\mathfrak{d}^{\prime} \mathrm{i}(p)\right) \quad \text { otherwise }
\end{aligned}
$$

from Lemma 41 in mind. For $f$, we obtain

$$
\begin{aligned}
f\left(\mathfrak{d}^{\prime} r^{m}\right) & =f\left(\mathrm{i} \pi \mathfrak{d}^{\prime} r^{m}+\sum_{n \in \mathbb{Z}} m^{\prime}\left(r^{m}, s^{n}\right) s^{n}\right) \stackrel{43}{=} f\left(\mathrm{i} \pi \mathfrak{d}^{\prime} r^{m}+m^{\prime}\left(r^{m}, s^{m}\right) s^{m}\right) \\
& =\pi \mathbf{i} \pi \mathfrak{d}^{\prime} r^{m}-0+0-m^{\prime}\left(r^{m}, s^{m}\right) m^{\prime}\left(r^{m}, s^{m}\right) \pi\left(\mathfrak{d}^{\prime} r^{m}\right) \\
& =\pi \mathfrak{d}^{\prime} r^{m}-\pi \mathfrak{d}^{\prime} r^{m}=0=\mathfrak{d} 0 \\
& =\mathfrak{d} f\left(r^{m}\right), \\
\mathfrak{d} f\left(s^{m}\right) & =\mathfrak{d}\left(-m^{\prime}\left(r^{m}, s^{m}\right) \pi \mathfrak{d}^{\prime} r^{m}\right) \stackrel{41}{=} \pi \mathfrak{d}^{\prime}\left(-m^{\prime}\left(r^{m}, s^{m}\right) i \pi \mathfrak{d}^{\prime} r^{m}\right) \\
& =-m^{\prime}\left(r^{m}, s^{m}\right) \pi \mathfrak{d}^{\prime} \mathfrak{i} \pi \mathfrak{d}^{\prime} r^{m} \\
& =-m^{\prime}\left(r^{m}, s^{m}\right) \pi \mathfrak{d}^{\prime}\left(\mathfrak{d}^{\prime} r^{m}-\sum_{n \in \mathbb{Z}} m^{\prime}\left(r^{m}, s^{n}\right) s^{n}\right) \\
& \stackrel{43}{=}-m^{\prime}\left(r^{m}, s^{m}\right)\left(\pi \mathfrak{d}^{\prime} \mathfrak{d}^{\prime} r^{m}-m^{\prime}\left(r^{m}, s^{m}\right) \pi \mathfrak{d}^{\prime} s^{m}\right) \\
& =\pi \mathfrak{d}^{\prime} s^{m} \\
& =f\left(\mathfrak{d}^{\prime} s^{m}\right) .
\end{aligned}
$$


For $p \neq r^{m}, s^{m}$ and $m \in \mathbb{Z}$, we obtain

$$
\begin{array}{rl}
f\left(\mathfrak{d}^{\prime} p\right)= & f\left(\mathrm{i} \pi \mathfrak{d}^{\prime} p+\sum_{n \in \mathbb{Z}} m^{\prime}\left(p, r^{n}\right) r^{n}+m^{\prime}\left(p, s^{n}\right) s^{n}\right) \\
= & \pi \mathrm{i} \pi \mathfrak{d}^{\prime} p-\pi\left(\sum_{l \in \mathbb{Z}} m^{\prime}\left(r^{l}, s^{l}\right)\left\langle\mathrm{i} \pi \mathfrak{d}^{\prime} p, s^{l}\right\rangle \mathfrak{d}^{\prime} r^{l}\right) \\
& +\pi\left(\sum_{n \in \mathbb{Z}} m^{\prime}\left(p, r^{n}\right) r^{n}\right) \\
& -\pi\left(\sum_{l \in \mathbb{Z}} m^{\prime}\left(r^{l}, s^{l}\right)\left\langle\sum_{n \in \mathbb{Z}} m^{\prime}\left(p, r^{n}\right) r^{n}, s^{l}\right\rangle \mathfrak{d}^{\prime} r^{l}\right) \\
& +\pi\left(\sum_{n \in \mathbb{Z}} m^{\prime}\left(p, s^{n}\right) s^{n}\right) \\
& -\pi\left(\sum_{l \in \mathbb{Z}} m^{\prime}\left(r^{l}, s^{l}\right)\left\langle\sum_{n \in \mathbb{Z}} m^{\prime}\left(p, s^{n}\right) s^{n}, s^{l}\right\rangle \mathfrak{d}^{\prime} r^{l}\right) \\
= & \pi \mathrm{i} \pi \mathfrak{d}^{\prime} p-0+0-0+0-\sum_{l \in \mathbb{Z}} m^{\prime}\left(p, s^{l}\right) m^{\prime}\left(r^{l}, s^{l}\right) \pi \mathfrak{d}^{\prime} r^{l} \\
= & \pi \mathfrak{d}^{\prime} p-\sum_{l \in \mathbb{Z}} m^{\prime}\left(p, s^{l}\right) m^{\prime}\left(r^{l}, s^{l}\right) \pi \mathfrak{d}^{\prime} r^{l} \\
= & \pi \mathfrak{d}^{\prime} \mathrm{i} \pi p-\sum_{l \in \mathbb{Z}} m^{\prime}\left(p, s^{l}\right) m^{\prime}\left(r^{l}, s^{l}\right) \pi \mathfrak{d}^{\prime} r^{l} \\
\underline{41} & \mathfrak{d} \pi p \\
= & \mathfrak{d} f(p) .
\end{array}
$$

Now we extend the definition of $m^{\prime}(p, q)$ etc. by linearity from primary points to elements of $\mathfrak{C}_{*}^{\prime}$, i.e., $m^{\prime}\left(\sum_{j} p_{j}, q\right):=\sum_{j} m^{\prime}\left(p_{j}, q\right)$, and consider $g$ :

Case $\mu(i(p), r)=0$ : We first show

$$
i \pi \mathfrak{d}^{\prime} g(p)=\mathfrak{d}^{\prime} g(p)
$$

which follows from $\left\langle\mathfrak{d}^{\prime} g(p), r^{m}\right\rangle=0$ due to $\mu\left(\mathrm{i}(p), r^{m}\right)=0$ for $m \in \mathbb{Z}$ and

$$
\begin{aligned}
\left\langle\mathfrak{d}^{\prime} g(p), s^{m}\right\rangle & =m^{\prime}\left(g(p), s^{m}\right)=m^{\prime}\left(i(p)-\sum_{n \in \mathbb{Z}} m^{\prime}\left(r^{n}, s^{n}\right) m^{\prime}\left(\mathrm{i}(p), s^{n}\right) r^{n}, s^{m}\right) \\
& =m^{\prime}\left(\mathrm{i}(p), s^{m}\right)-\sum_{n \in \mathbb{Z}} m^{\prime}\left(r^{n}, s^{n}\right) m^{\prime}\left(\mathrm{i}(p), s^{n}\right) m^{\prime}\left(r^{n}, s^{m}\right)
\end{aligned}
$$




$$
\begin{aligned}
& \stackrel{43}{=} m^{\prime}\left(\mathrm{i}(p), s^{m}\right)-m^{\prime}\left(r^{m}, s^{m}\right) m^{\prime}\left(\mathrm{i}(p), s^{m}\right) m^{\prime}\left(r^{m}, s^{m}\right) \\
& =m^{\prime}\left(\mathrm{i}(p), s^{m}\right)-m^{\prime}\left(\mathrm{i}(p), s^{m}\right) \\
& =0 .
\end{aligned}
$$

Now we obtain

$$
\begin{aligned}
g(\mathfrak{d} p) \stackrel{\mu(i(p), r)=0}{=} \mathrm{i}(\mathfrak{d} p) \stackrel{41}{=} \mathrm{i} \pi \mathfrak{d}^{\prime}\left(\mathrm{i}(p)-\sum_{n \in \mathbb{Z}} m^{\prime}\left(\mathrm{i}(p), s^{n}\right) m^{\prime}\left(r^{n}, s^{n}\right) r^{n}\right) \\
=\mathrm{i} \pi \mathfrak{d}^{\prime} g(p) \stackrel{45}{=} \mathfrak{d}^{\prime} g(p) .
\end{aligned}
$$

Case $\mu(i(p), r) \neq 0$ : First note

$$
m^{\prime}\left(\mathfrak{d}^{\prime} \mathrm{i}(p), s^{m}\right)=\left\langle\mathfrak{d}^{\prime}\left(\mathfrak{d}^{\prime} \mathrm{i}(p)\right), s^{m}\right\rangle=0
$$

and then compute

$$
\begin{aligned}
& g(\mathfrak{d} p) \stackrel{41}{=} g\left(\pi \mathfrak{d}^{\prime} \mathrm{i}(p)\right) \\
& =\mathrm{i} \pi \mathfrak{d}^{\prime} \mathrm{i}(p)-\sum_{n \in \mathbb{Z}} m^{\prime}\left(r^{n}, s^{n}\right) m^{\prime}\left(\mathrm{i} \pi \mathfrak{d}^{\prime} \mathrm{i}(p), s^{n}\right) r^{n} \\
& =\mathrm{i} \pi \mathfrak{d}^{\prime} \mathrm{i}(p)-\sum_{n \in \mathbb{Z}} m^{\prime}\left(r^{n}, s^{n}\right) m^{\prime}\left(\mathfrak{d}^{\prime} \mathrm{i}(p)\right. \\
& \left.-\sum_{l \in \mathbb{Z}} m^{\prime}\left(\mathrm{i}(p), r^{l}\right) r^{l}-m^{\prime}\left(\mathrm{i}(p), s^{l}\right) s^{l}, s^{n}\right) r^{n} \\
& =\mathrm{i} \pi \mathfrak{d}^{\prime} \mathrm{i}(p)-\sum_{n \in \mathbb{Z}} m^{\prime}\left(r^{n}, s^{n}\right)\left(m^{\prime}\left(\mathfrak{d}^{\prime} \mathrm{i}(p), s^{n}\right)\right. \\
& \left.-\sum_{l \in \mathbb{Z}} m^{\prime}\left(\mathrm{i}(p), r^{l}\right) m^{\prime}\left(r^{l}, s^{n}\right)-0\right) r^{n} \\
& \stackrel{(46)}{=} \mathrm{i} \pi \mathfrak{d}^{\prime} \mathrm{i}(p)+\sum_{n \in \mathbb{Z}} m^{\prime}\left(r^{n}, s^{n}\right) m^{\prime}\left(\mathrm{i}(p), r^{n}\right) m^{\prime}\left(r^{n}, s^{n}\right) r^{n} \\
& =\mathrm{i} \pi \mathfrak{d}^{\prime} \mathrm{i}(p)+\sum_{n \in \mathbb{Z}} m^{\prime}\left(\mathrm{i}(p), r^{n}\right) r^{n}
\end{aligned}
$$

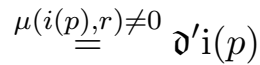

$$
\begin{aligned}
& \mu(i(p), r) \neq 0 \quad \mathfrak{d}^{\prime}(g(p)) .
\end{aligned}
$$

Since $\pi$ and $i$ are $\mathbb{Z}$-equivariant so are $f$ and $g$.

Now we show that $f$ and $g$ induce isomorphisms between the homologies of $\left(\mathfrak{C}_{*}^{\prime}, \mathfrak{d}^{\prime}\right)$ and $\left(\mathfrak{C}_{*}, \mathfrak{d}\right)$.

Theorem 47. The homologies of $\left(\mathfrak{C}_{*}^{\prime}, \mathfrak{d}^{\prime}\right)$ and $\left(\mathfrak{C}_{*}, \mathfrak{d}\right)$ are isomorphic. 
Proof. For $f$ and $g$ from Lemma 44, we show that $f_{*}: H\left(\mathfrak{C}_{*}^{\prime}, \mathfrak{d}^{\prime}\right) \rightarrow H\left(\mathfrak{C}_{*}, \mathfrak{d}\right)$ and $g_{*}: H\left(\mathfrak{C}_{*}, \mathfrak{d}\right) \rightarrow H\left(\mathfrak{C}_{*}^{\prime}, \mathfrak{d}^{\prime}\right)$ are inverse to each other. It is enough to show $f \circ g \simeq \operatorname{Id}_{\mathfrak{C}_{*}}$ and $g \circ f \simeq \operatorname{Id}_{\mathfrak{C}_{*}^{\prime \prime}}$ where $\simeq$ stands for homotopic by a chain homotopy. $f \circ g:\left(\mathfrak{C}_{*}, \mathfrak{d}\right) \rightarrow\left(\mathfrak{C}_{*}, \mathfrak{d}\right)$ is even the identity:

$$
\begin{aligned}
f(g(p)) & =f(\mathrm{i}(p))-\sum_{n \in \mathbb{Z}} m^{\prime}\left(r^{n}, s^{n}\right) m^{\prime}\left(\mathrm{i}(p), s^{n}\right) f\left(r^{n}\right)=f(i(p)) \\
& =\pi \mathrm{i}(p)-\pi\left(\sum_{n \in \mathbb{Z}} m^{\prime}\left(r^{n}, s^{n}\right)\left\langle\mathrm{i}(p), s^{n}\right\rangle \mathfrak{d}^{\prime} r^{n}\right)=\pi \mathrm{i}(p)=\operatorname{Id}_{\mathfrak{C}_{*}}(p) .
\end{aligned}
$$

Unfortunately, this is not true for $g \circ f$. However, we can find a chain homotopy $h:\left(\mathfrak{C}_{*}^{\prime}, \mathfrak{d}^{\prime}\right) \rightarrow\left(\mathfrak{C}_{*+1}^{\prime}, \mathfrak{d}^{\prime}\right)$ satisfying $g \circ f-\operatorname{Id}_{\mathfrak{C}_{*}^{\prime}}=h \circ \mathfrak{d}^{\prime}+\mathfrak{d}^{\prime} \circ h$. Choose

$$
h(p):=-\sum_{n \in \mathbb{Z}}\left\langle s^{n}, p\right\rangle m^{\prime}\left(r^{n}, s^{n}\right) r^{n}
$$

and compute for $m \in \mathbb{Z}$

$$
\begin{aligned}
\left(h \circ \mathfrak{d}^{\prime}\right. & \left.+\mathfrak{d}^{\prime} \circ h\right)\left(r^{m}\right) \\
& =-\sum_{n \in \mathbb{Z}}\left\langle s^{n}, \mathfrak{d}^{\prime} r^{m}\right\rangle m^{\prime}\left(r^{n}, s^{n}\right) r^{n}-\mathfrak{d}^{\prime}\left(\sum_{n \in \mathbb{Z}}\left\langle s^{n}, r^{m}\right\rangle m^{\prime}\left(r^{n}, s^{n}\right) r^{n}\right) \\
& =-\sum_{n \in \mathbb{Z}} m^{\prime}\left(r^{m}, s^{n}\right) m^{\prime}\left(r^{n}, s^{n}\right) r^{n} \\
& \stackrel{43}{=}-m^{\prime}\left(r^{m}, s^{m}\right) m^{\prime}\left(r^{m}, s^{m}\right) r^{m} \\
& =-r^{m}
\end{aligned}
$$

and

$$
\begin{aligned}
\left(h \circ \mathfrak{d}^{\prime}\right. & \left.+\mathfrak{d}^{\prime} \circ h\right)\left(s^{m}\right) \\
& =-\sum_{n \in \mathbb{Z}}\left\langle s^{n}, \mathfrak{d}^{\prime} s^{m}\right\rangle m^{\prime}\left(r^{n}, s^{n}\right) r^{n}-\sum_{n \in \mathbb{Z}}\left\langle s^{n}, s^{m}\right\rangle m^{\prime}\left(r^{n}, s^{n}\right) \mathfrak{d}^{\prime} r^{n} \\
& =-m^{\prime}\left(r^{m}, s^{m}\right) \mathfrak{d}^{\prime} r^{m}
\end{aligned}
$$

and for $p \neq r^{m}, s^{m}$ for $m \in \mathbb{Z}$

$$
\begin{aligned}
\left(h \circ \mathfrak{d}^{\prime}\right. & \left.+\mathfrak{d}^{\prime} \circ h\right)(p) \\
& =-\sum_{n \in \mathbb{Z}}\left\langle s^{n}, \mathfrak{d}^{\prime} p\right\rangle m^{\prime}\left(r^{n}, s^{n}\right) r^{n}-\mathfrak{d}^{\prime}\left(\sum_{n \in \mathbb{Z}}\left\langle s^{n}, p\right\rangle m^{\prime}\left(r^{n}, s^{n}\right) r^{n}\right) \\
& =-\sum_{n \in \mathbb{Z}} m^{\prime}\left(p, s^{n}\right) m^{\prime}\left(r^{n}, s^{n}\right) r^{n} .
\end{aligned}
$$

On the other hand, we obtain

$$
\left(g \circ f-\operatorname{Id}_{\mathfrak{C}_{*}^{\prime}}\right)\left(r^{m}\right)=g\left(f\left(r^{m}\right)\right)-r^{m}=-r^{m}
$$


and

$$
\begin{aligned}
(g \circ f & \left.-\operatorname{Id}_{\mathfrak{C}_{*}^{\prime \prime}}\right)\left(s^{m}\right) \\
& =g\left(\pi\left(s^{m}\right)-\pi\left(\sum_{n \in \mathbb{Z}} m^{\prime}\left(r^{n}, s^{n}\right)\left\langle s^{m}, s^{n}\right\rangle \mathfrak{d}^{\prime} r^{n}\right)\right)-s^{m} \\
& =g\left(-m^{\prime}\left(r^{m}, s^{m}\right) \pi \mathfrak{d}^{\prime} r^{m}\right)-s^{m} \\
& =-m^{\prime}\left(r^{m}, s^{m}\right) g\left(\pi \mathfrak{d}^{\prime} r^{m}\right)-s^{m} \\
& =-m^{\prime}\left(r^{m}, s^{m}\right)\left(\mathrm{i} \pi \mathfrak{d}^{\prime} r^{m}-\sum_{n \in \mathbb{Z}} m^{\prime}\left(r^{n}, s^{n}\right) m^{\prime}\left(\mathrm{i} \pi \mathfrak{d}^{\prime} r^{m}, s^{n}\right) r^{n}\right)-s^{m} \\
& =-m^{\prime}\left(r^{m}, s^{m}\right) \mathrm{i} \pi \mathfrak{d}^{\prime} r^{m}-s^{m} \\
& =-m^{\prime}\left(r^{m}, s^{m}\right)\left(\mathfrak{d}^{\prime} r^{m}-\sum_{n \in \mathbb{Z}} m^{\prime}\left(r^{m}, s^{n}\right) s^{n}\right)-s^{m} \\
& \stackrel{43}{=}-m^{\prime}\left(r^{m}, s^{m}\right) \mathfrak{d}^{\prime} r^{m}+m^{\prime}\left(r^{m}, s^{m}\right) m^{\prime}\left(r^{m}, s^{m}\right) s^{m}-s^{m} \\
& =-m^{\prime}\left(r^{m}, s^{m}\right) \mathfrak{d}^{\prime} r^{m}
\end{aligned}
$$

and for $p \neq r^{m}, s^{m}$ for $m \in \mathbb{Z}$

$$
\begin{aligned}
\left(g \circ f-\operatorname{Id}_{\mathfrak{C}_{*}^{\prime}}\right)(p) & =g\left(\pi(p)-\sum_{n \in \mathbb{Z}} m^{\prime}\left(r^{n}, s^{n}\right)\left\langle p, s^{n}\right\rangle \pi \mathfrak{d}^{\prime} r^{n}\right)-p \\
& =g(\pi(p))-p \\
& =\mathrm{i} \pi(p)-\sum_{n \in \mathbb{Z}} m^{\prime}\left(r^{n}, s^{n}\right) m^{\prime}\left(\mathrm{i} \pi(p), s^{n}\right) r^{n}-p \\
& =-\sum_{n \in \mathbb{Z}} m^{\prime}\left(r^{n}, s^{n}\right) m^{\prime}\left(p, s^{n}\right) r^{n} .
\end{aligned}
$$

Comparing the results yields $g \circ f-\operatorname{Id}_{\mathfrak{C}_{*}^{\prime}}=h \circ \mathfrak{d}^{\prime}+\mathfrak{d}^{\prime} \circ h$ which proves the claim.

Moreover, note that also the chain homotopy $h$ commutes with the $\mathbb{Z}$ action on the chain complexes. Now we divide by the $\mathbb{Z}$-action. Define $C_{*}$ and $C_{*}^{\prime}$ analogously to $\mathfrak{C}_{*}$ and $\mathfrak{C}_{*}^{\prime}$. Since $f, g$ and $h$ commute with the $\mathbb{Z}$ action on the chain complexes they pass to $C_{*}$ and $C_{*}^{\prime}$ and we obtain

Theorem 48. The homologies of $\left(C_{*}, \partial\right)$ and $\left(C_{*}^{\prime}, \partial^{\prime}\right)$ are isomorphic, i.e., primary moves leave the primary Floer homology invariant.

5.5. Invariance under mixed moves. The invariance under mixed moves will be reduced to the invariance under primary and secondary moves. If not stated otherwise, we will work with the lifted tangles on the universal cover.

Now we want to investigate how mixed moves look like. If a $(r, s)$-move flips a primary points $p$ secondary the segments $] p, x[u$ and $] p, x[s$ have to 
(i)

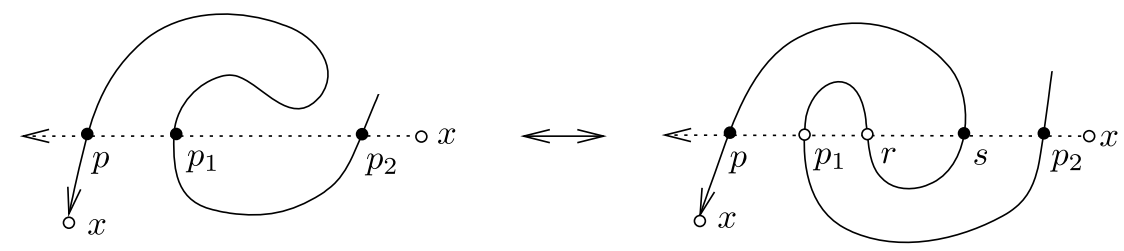

(ii)

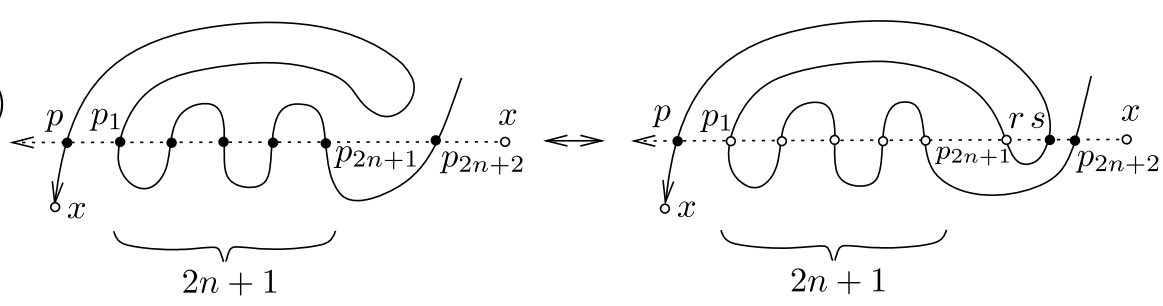

Figure 13. Mixed $(r, s)$-moves with one flip in (i) and $2 \mathrm{n}+1$ flips in (ii).

intersect after the move. In particular, $r, s$ and $p$ have to lie in the same pair of branches. Since the (un)stable manifolds are free of self-intersections a mixed move always takes place within a fixed frame, i.e., the mixed move cannot 'overlap' into another iterate of the frame. With Remark 18 in mind, mixed moves look as sketched in Figure 13 (where $p_{2 n+1}$ still lies in the frame induced by $p$ ). Mixed moves come along with $2 n+1$ flips and are called simple if $n=0$.

Before we consider the invariance under mixed moves we note the following. Without the condition ' $\cdots \cap \mathcal{H}_{[x]}$ ' in the definition of 'primary', primary Floer homology would not be invariant: Consider Figure 14 where a move circles around genus. Assume for sake of simplicity that only the branches containing $p$ intersect. In our convention, the move is secondary and thus leaves the homology invariant. Dropping ' $\cdots \cap \mathcal{H}_{[x]}$ ' is equivalent to using the contractible semi-primary points as generators of the chain complex. Before the move, $p$ and $q$ are contractible and semi-primary, but after the move $q$ is no longer semi-primary. The generated $r$ is secondary and $s$ semi-primary, but not contractible. Thus, it is excluded as generator. Before the move we obtain $H_{-1}=\mathbb{Z}\langle p\rangle$ and $H_{-2}=\mathbb{Z}\langle q\rangle$ and $H_{*}=0$ for $n \neq-1,-2$. However, after the move there is only $p$ left as generator. Thus, $H_{-1}=\mathbb{Z}\langle p\rangle$ and $H_{*}=0$ otherwise. This phenomenon inspires the definition of semi-primary Floer homology in Theorem 57.

Proposition 49. Primary Floer homology stays invariant under simple mixed moves. 


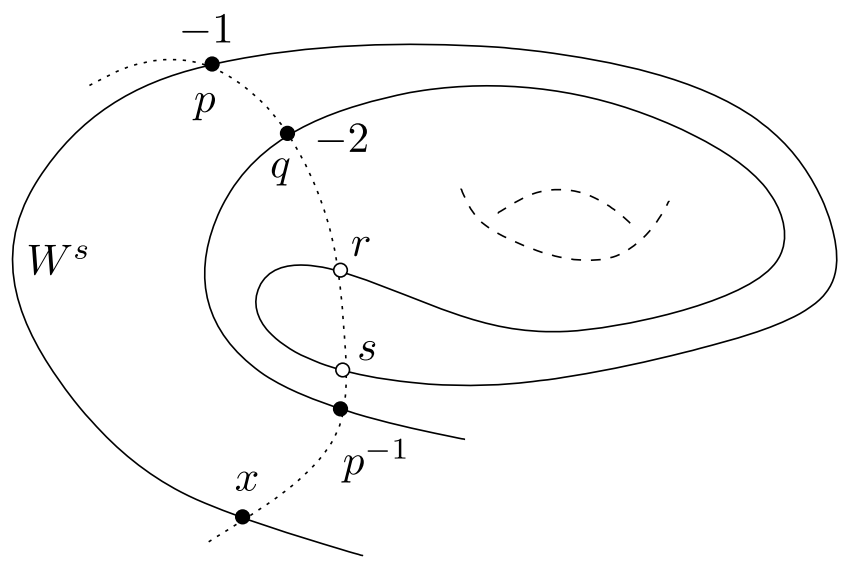

Figure 14. Arising of nontrivial homotopy classes.

Proof. Consider Figure 13 (i): the simple mixed $(r, s)$-move can be recognized as an identification followed by the secondary $\left(p_{1}, r\right)$-move. Since both leave the homology invariant so does the simple mixed move. An explicit chain complex isomorphism is given by $f:\left(C_{*}, \partial\right) \longrightarrow\left(C_{*}^{\prime}, \partial^{\prime}\right), a \mapsto a$ for $a \in \tilde{\mathcal{H}}_{p r} \backslash\left\{\left\langle p_{1}\right\rangle\right\}$ and $\left\langle p_{1}\right\rangle \mapsto\langle s\rangle$.

Now we consider the invariance under arbitrary mixed moves.

Theorem 50. Primary Floer homology is invariant under mixed moves.

Proof. For simple mixed moves, the claim was already proven in Proposition 49. Now consider Figure 13 (ii). The mixed move can be recognized as a sequence of primary moves $\left(p_{2}, p_{3}\right), \ldots,\left(p_{2 n}, p_{2 n+1}\right)$, followed by a simple mixed $(r, s)$-move and a sequence of secondary moves $\left(p_{2}, p_{3}\right), \ldots$, $\left(p_{2 n}, p_{2 n+1}\right)$. This yields the claim.

5.6. The proof of Proposition 35. Let $\varphi \in \operatorname{Diff}_{\omega}(M)$ with hyperbolic $x \in \operatorname{Fix}(\varphi)$. Let $(x, \varphi)$ be csi and let all primary points be transverse. First we generalize Lemma 37.

Lemma 51. (1) Let $\hat{\varphi} \in \operatorname{Diff}_{\omega}(M)$ be a small perturbation of $\varphi$ and $\hat{x}$ the continuation of $x$. Let $p_{\varphi} \in \mathcal{H}_{p r}(\varphi)$ be primary and let $p_{\varphi}$ persist as transverse homoclinic points $p_{\hat{\varphi}}$, but nonprimary. Then there is $q \in$ $\mathcal{H}_{p r}(\hat{\varphi})$ which is no continuation of any primary point of $\varphi$.

(2) Let $(x, \varphi)$ be csi and let all primary points be transverse. Then for sufficiently small perturbations $\hat{\varphi} \in \operatorname{Diff}_{\omega}(M)$ of $\varphi$, all primary points remain transverse and no primary points arise or vanish.

Proof. First item: We work with the lifted tangles of $\varphi$ and $\hat{\varphi}$, but we drop the tilde for sake of readability. The segments $\left[x, p_{\varphi}\right]_{i}$ and $\left[\hat{x}, p_{\hat{\varphi}}\right]_{i}$ are close. 


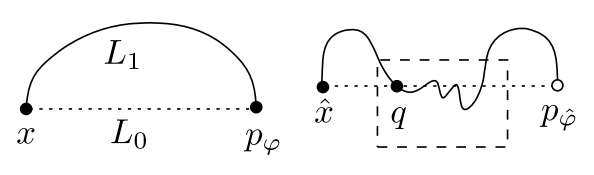

(i) (ii)

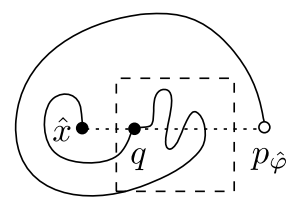

(iii)

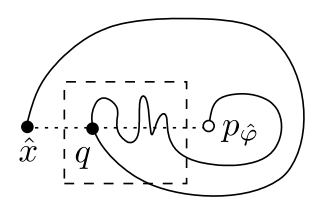

(iv)

Figure 15. Causes for primary-secondary flips.

Since $p_{\varphi}$ is primary $] x, p_{\varphi}[u \cap] x, p_{\varphi}\left[s=\emptyset\right.$. However, $p_{\hat{\varphi}}$ is nonprimary, thus ]$\hat{x}, p_{\hat{\varphi}}[u \cap] \hat{x}, p_{\hat{\varphi}}\left[s \neq \emptyset . \hat{x}\right.$ and $p_{\hat{\varphi}}$ remain transverse. Figure 15 (ii)-(iv) lists the three types that prevent $p_{\hat{\varphi}}$ to be primary. In all three cases, there is a primary $q \in] \hat{x}, p_{\hat{\varphi}}[u \cap] \hat{x}, p_{\hat{\varphi}}[s$ which has no corresponding point in ]$x, p_{\varphi}[u \cap] x, p_{\varphi}\left[s\right.$ and thus in $\mathcal{H}_{p r}(\varphi)$.

Second item: Since all primary points of $\varphi$ are transverse they persist at least as transverse intersection points for small perturbations. Any primarysecondary flip would require the rise of a new primary point. However, primary points only arise in frames and the compactness of the frame prevents this for sufficiently small perturbations.

Thus also in this generalized situation, a primary-secondary flip is coupled with the rise of a new primary point. Now we generalize Proposition 39.

Lemma 52. Let $\varphi \in \operatorname{Diff}_{\omega}(M)$ be csi with $x \in \operatorname{Fix}(\varphi)$ hyperbolic and all primary points transverse. Let $\hat{\varphi} \in \operatorname{Diff}_{\omega}(M)$ be small perturbation of $\varphi$ such that all primary points persist transverse. Consider primary $p_{\varphi}$ and $q_{\varphi}$ with $\mu\left(p_{\varphi}, q_{\varphi}\right)=1$ and denote their continuation by $p_{\hat{\varphi}}$ and $q_{\hat{\varphi}}$. Then $m\left(p_{\varphi}, q_{\varphi}\right)=m\left(p_{\hat{\varphi}}, q_{\hat{\varphi}}\right)$.

Proof. For simplicity, abbreviate $p:=p_{\hat{\varphi}}$ and $q:=q_{\hat{\varphi}}$ Clearly $\mu\left(p_{\varphi}, q_{\varphi}\right)=$ $\mu(p, q)$ and if $m\left(p_{\varphi}, q_{\varphi}\right) \neq 0 \neq m(p, q)$ then their signs coincide. Thus it is enough to show $\mathcal{M}\left(p_{\varphi}, q_{\varphi}\right) \neq \emptyset$ if and only if $\mathcal{M}(p, q) \neq \emptyset$. We will work on the universal cover with the lifted tangles.

We have to check if the proof of Proposition 39 carries over to our more general situation. Let $\mathcal{M}\left(p_{\varphi}, q_{\varphi}\right) \neq \emptyset$ and assume $\mathcal{M}(p, q)=\emptyset$, i.e. ]$p, q[u \cap] p, q\left[{ }_{s} \neq \emptyset\right.$.

For that, we have to admit perturbations as in the dashed boxes in Figure 15 and check a generalization of Figure 11 etc. which yields the claim.

Proof of Proposition 35. For sufficiently small perturbations, the primary points persist by Lemma 51 (2). Thus, the generator set of primary homoclinic chain complex stays unchanged. Moreover, the boundary operator persists due to Lemma 52. Thus the homology remains unchanged. 


\section{Dynamics and homoclinic Floer theory}

6.1. Conjugacy. Since conjugacy does not affect the intersection behaviour of homoclinic tangles one expects primary Floer homology to be invariant under conjugacy. Nevertheless, one has to be a little careful. If one is only interested in the topological information, then it is enough to have a homeomorphisms $h$ conjugating $\varphi, \psi \in \operatorname{Diff}_{\omega}(M)$, i.e., $\varphi \circ h=h \circ \psi$ : If $x \in \operatorname{Fix}(\psi)$ then $h(x) \in \operatorname{Fix}(\varphi)$ and $H_{*}(x, \psi)=H_{*}(h(x), \varphi)$ since $h\left(W^{i}(x, \psi)\right)=W^{i}(h(x), \varphi)$ for $i \in\{s, u\}$.

But if symplectic properties should be preserved (as for example the symplectic volume $\int v^{*} \omega$ of an immersion $v$ ) we have to require $h$ to be symplectic.

6.2. $\operatorname{rk} \boldsymbol{H}_{*}(\boldsymbol{x}, \boldsymbol{\varphi}) \leq \operatorname{rk} \boldsymbol{H}_{*}\left(\boldsymbol{x}, \boldsymbol{\varphi}^{\boldsymbol{n}}\right)$. Now denote by $\left\langle p_{1}\right\rangle, \ldots,\left\langle p_{k}\right\rangle$ the generators of $C_{*}(x, \varphi)$ and set $p_{i}^{j}:=\varphi^{j}\left(p_{i}\right)$. For $n \in \mathbb{N}_{0}$, we have $W_{i}^{\varphi}=$ $W_{i}^{\varphi^{n}}$ for $i \in\{s, u\}$ and $W_{i}^{\varphi}=W_{j}^{\varphi^{-n}}$ for $i \neq j \in\{s, u\}$. Note that the number of equivalence classes multiplies: $C_{*}\left(x, \varphi^{n}\right)$ is generated by $\left\langle p_{1}^{0}\right\rangle, \ldots,\left\langle p_{k}^{0}\right\rangle,\left\langle p_{1}^{1}\right\rangle, \ldots,\left\langle p_{k}^{n-1}\right\rangle$. Abbreviate $\mathbb{Z}_{n}:=\mathbb{Z} / n \mathbb{Z}=\{\overline{0}, \overline{1}, \ldots, \overline{n-1}\}$ and set $\varphi_{*}^{l}=\bar{l}$. There is a $\mathbb{Z}_{n}$-action on the generators via

$$
\mathbb{Z}_{n} \times C_{*}\left(x, \varphi^{n}\right) \rightarrow C_{*}\left(x, \varphi^{n}\right), \quad \varphi_{*}^{l} \cdot\left\langle p_{i}^{j}\right\rangle:=\left\langle p_{i}^{j+l \bmod n}\right\rangle=\left\langle\varphi^{l}\left(p_{i}^{j}\right)\right\rangle
$$

and extend it by linearity to the complex. We notice $\varphi_{*}^{l} \cdot\left(\partial\left\langle p_{i}^{j}\right\rangle\right)=\partial\left\langle\varphi^{l}\left(p_{i}^{j}\right)\right\rangle$ such that the $\mathbb{Z}_{n}$-action descends to homology. If we use the $m(p, q)$-signs we assume $\mathbb{Q}$-coefficients and so we do for $n(p, q)$-signs if $\varphi$ is $W$-orientation preserving $\varphi$ and $n \in \mathbb{N}_{0}$. In the orientation reversing case, assume $\mathbb{Z}_{2}$ as coefficient ring for the $n(p, q)$-signs if $n=2 m+1 \in \mathbb{N}$ odd. Then Theorem 25 allows us to treat simultaneously also negative exponents and we define

$$
\begin{aligned}
& f: C_{*}\left(x, \varphi^{n} ; \mathbb{K}\right) \simeq C^{-*}\left(x, \varphi^{-n}, \mathbb{K}\right) \rightarrow C_{*}(x, \varphi ; \mathbb{K}), \quad f\left(\left\langle p_{i}^{j}\right\rangle\right):=\left\langle p_{i}\right\rangle, \\
& g: C_{*}(x, \varphi ; \mathbb{K}) \rightarrow C_{*}\left(x, \varphi^{n} ; \mathbb{K}\right) \simeq C^{-*}\left(x, \varphi^{-n} ; \mathbb{K}\right), \quad g\left(\left\langle p_{i}\right\rangle\right):=\frac{1}{n} \sum_{j=0}^{n-1}\left\langle p_{i}^{j}\right\rangle
\end{aligned}
$$

where $\mathbb{K}$ stands for the suitable coefficient ring. $f$ and $g$ are chain maps and we compute $f \circ g=\operatorname{Id}_{C_{*}(x, \varphi ; \mathbb{K})}$. Denote by $g_{*}$ and $f_{*}$ the induced maps on the (co)homology.

Proposition 53. $g_{*}$ is injective, $f_{*}$ surjective and

$$
\operatorname{rk} H_{*}(x, \varphi ; \mathbb{K}) \leq \operatorname{rk} H_{*}\left(x, \varphi^{n} ; \mathbb{K}\right)=\operatorname{rk} H^{-*}\left(x, \varphi^{-n} ; \mathbb{K}\right) .
$$

The difference is measured by the long exact sequence

$$
\cdots \rightarrow H_{l}(\operatorname{ker} f ; \mathbb{K}) \rightarrow H_{l}\left(x, \varphi^{n} ; \mathbb{K}\right) \rightarrow H_{l}(x, \varphi ; \mathbb{K}) \rightarrow H_{l-1}(\operatorname{ker} f, \mathbb{K}) \rightarrow \cdots
$$

Proof. We drop the coefficient ring $\mathbb{K}$ in the notation in favour of better readability. $f \circ g=\operatorname{Id}_{C_{*}(x, \varphi)}$ implies the injectivity of $g_{*}$ and surjectivity of 
$f_{*}$ which yield the dimension estimates. The range of $g$ are the invariants under the $\mathbb{Z}_{n}$-action and the kernel of $f$ the coinvariants, which are both subcomplexes of $C_{*}\left(x, \varphi^{n}\right)$. We obtain the short exact sequence of chain complexes

$$
\left((\operatorname{ker} f)_{*}, \partial\right) \hookrightarrow\left(C_{*}\left(x, \varphi^{n}\right), \partial\right) \rightarrow\left(\frac{C_{*}\left(x, \varphi^{n}\right)}{(\operatorname{ker} f)_{*}}, \bar{\partial}\right)
$$

where $\bar{\partial}$ is induced by the projection. Moreover

$$
h:\left(\frac{C_{*}\left(x, \varphi^{n}\right)}{(\operatorname{ker} f)_{*}}, \bar{\partial}\right) \rightarrow\left(\operatorname{Im}(g)_{*}, \partial\right), \quad[c] \mapsto \sum_{l=0}^{n-1} \varphi_{*}^{l}(c)
$$

is an isomorphism and satisfies $h \circ \bar{\partial}=\partial \circ h$, thus an isomorphism of chain complexes. Since also $g: C_{*}(x, \varphi) \rightarrow \operatorname{Im}(g)_{*}$ is an isomorphism of chain complexes we obtain by means of the long exact sequence of (54)

$$
\cdots \rightarrow H_{l}(\operatorname{ker} f) \rightarrow H_{l}\left(x, \varphi^{n}\right) \rightarrow H_{l}(x, \varphi) \rightarrow H_{l-1}(\operatorname{ker} f) \rightarrow \cdots
$$

Now let us discuss under which circumstances we might have equality in Proposition 53. We call a smooth Hamiltonian function $H: \mathbb{R} \times M \rightarrow$ $\mathbb{R}$ (with compact support) normalized if $\int_{M} H_{t} d$ vol $=0$ for all $t$ where $H_{t}:=H(t, \cdot)$. Let $X$ be its nonautonomous vector field. Denote by $\varphi_{\left(t, t_{0}\right)}$ the nonautonomous flow of $\dot{z}(t)=X(t, z(t))$ starting at time $t_{0}$, i.e., $\varphi:=\varphi_{(1,0)}$ is the usual time-1 map. If we assume in addition $H(t, \cdot)=H(t+1, \cdot)$ then $\varphi_{(n, 0)}=\varphi_{(1,0)}^{n}=\varphi^{n}$ and $\varphi$ and $\varphi^{n}$ are joint by the isotopy $\tau \mapsto \varphi_{(1+(n-1) \tau, 0)}$. Changing the parametrization of a Hamiltonian path is easy: Given $\tau \mapsto$ $\psi_{(\tau, 0)}$ with Hamiltonian $F(t, z)$, we obtain $\tau \mapsto \psi_{(b(\tau), 0)}$ using $b^{\prime}(t) F(b(t), z)$ as Hamiltonian.

Conversely, Banyaga [Ba] proved that for every path of Hamiltonian diffeomorphisms $\tau \mapsto \psi_{\tau}$, there is a normalized Hamiltonian having $\psi_{\tau}=\psi_{(\tau, 0)}$ as nonautonomous flow. Therefore we conclude

Corollary 55. Equality for Hamiltonian diffeomorphisms in Proposition 53 is tied to the question of invariance of primary Floer homology under Hamiltonian isotopies, i.e., the question if Hamiltonian isotopies fulfil the requirements of Theorem 31. In Remark 36, we conjecture the answer to be affirmative.

If there is (conjecturally) equality for Hamiltonian diffeomorphisms in Proposition 53 we need to know how large the groups of Hamiltonian diffeomorphisms $\operatorname{Ham}(M, \omega) \subset \operatorname{Diff}_{\omega}(M)$ actually is. Assume $M$ to be closed and denote by $\operatorname{Diff}_{\omega}^{0}(M)$ the component of the identity in $\operatorname{Diff}_{\omega}(M)$. The difference between $\operatorname{Diff}_{\omega}^{0}(M)$ and $\operatorname{Ham}(M, \omega)$ is measured via

$$
\operatorname{Diff}_{\omega}^{0}(M) / \operatorname{Ham}(M, \omega)=H^{1}(M, \mathbb{R}) / \Gamma
$$


where $\Gamma \subset H^{1}(M, \mathbb{R})$ is the so-called flux group (cf. Polterovich [Pol2]). Thus for manifolds with vanishing first cohomology class, we have $\operatorname{Diff}_{\omega}^{0}(M)=$ $\operatorname{Ham}(M, \omega)$. Examples with $H(x, \varphi)<H\left(x, \varphi^{n}\right)$ might arise for nonHamiltonian symplectomorphisms, especially symplectomorphisms not isotopic to the identity.

Another interesting aspect is the relationship between $H\left(x, \varphi^{n}\right)$ and $\mathfrak{H}_{m}(x, \varphi)$. One might ask if actually $H\left(x, \varphi^{n}\right)$ might somehow converge to $\mathfrak{H}_{m}(x, \varphi)$. This turns out to be not true at least for Hamiltonian diffeomorphisms.

Proposition 56. There is $\varphi \in \operatorname{Ham}(M, \omega)$ with $H\left(x, \varphi^{n}\right) \neq \mathfrak{H}(x, \varphi) \forall n$.

Proof. Consider the homoclinic tangle in Figure 9 (a). There are exactly two distinct equivalence classes of primary points. Let us denote them by $\langle p\rangle$ and $\langle q\rangle$ and assume w.l.o.g. $\mu(\langle p\rangle)=-1$ and $\mu(\langle q\rangle)=-2$. Then $\partial\langle p\rangle=\langle q\rangle-$ $\langle q\rangle=0$ and $\partial\langle q\rangle=0$ and we obtain $H_{-1}(x, \varphi) \simeq \mathbb{Z}\langle p\rangle$ and $H_{-2}(x, \varphi) \simeq \mathbb{Z}\langle q\rangle$. Moreover, we calculate explicitly $H_{-1}\left(x, \varphi^{n}\right) \simeq \mathbb{Z} \simeq H_{-2}\left(x, \varphi^{n}\right)$ for $n \in \mathbb{N}$.

On the other hand, we compute $\mathfrak{C}_{-1}=\operatorname{Span}_{\mathbb{Z}}\left\{p^{l} \mid l \in \mathbb{Z}\right\}$ and $\mathfrak{C}_{-2}=$ $\operatorname{Span}_{\mathbb{Z}}\left\{q^{l} \mid l \in \mathbb{Z}\right\}$ and $\mathfrak{d} p^{l}=q^{l}-q^{l-1}$ and $\mathfrak{d} q^{l}=0$ for all $l \in \mathbb{Z}$. Thus $\mathfrak{H}_{-1}=$ 0 and $\mathfrak{H}_{-2}=\operatorname{Span}_{\mathbb{Z}}\left\{q^{n} \mid n \in \mathbb{Z}\right\} / \operatorname{Span}_{\mathbb{Z}}\left\{q^{n}+q^{n-1}\right\} \simeq \mathbb{Z}$ and therefore $\mathfrak{H}_{-1} \neq H_{-1}$.

6.3. $\operatorname{rk} \tilde{\boldsymbol{H}}_{*}(\boldsymbol{x}, \varphi)<\operatorname{rk} \tilde{\boldsymbol{H}}_{*}\left(\boldsymbol{x}, \varphi^{n}\right)$. In this section, we define a version of homoclinic Floer homology based on contractible semi-primary points, called semi-primary Floer homology. The construction is analogous to primary Floer homology except for the invariance property in Theorem 31. The weaker invariance property of semi-primary Floer homology allows a better sensitivity for the underlying symplectomorphism. For example, certain interactions of the tangle and the topology of the manifold are noticed to which primary Floer homology is oblivious. Moreover, semi-primary Floer homology distinguishes between $\varphi$ and $\varphi^{n}$ for certain symplectomorphisms $\varphi$.

Denote by $\mathcal{H}_{s}:=\left\{p \in \mathcal{H}_{[x]} \mid\right] x, p\left[{ }_{u} \cap\right] x, p\left[{ }_{s}=\emptyset\right\}$ the set of contractible semi-primary points and by $\tilde{\mathcal{H}}_{s}:=\mathcal{H}_{s} / \sim$ the set of contractible semi-primary equivalence classes where $p \sim q$ if and only if $p=q^{n}$ for some $n \in \mathbb{Z}$. As before, the equivalence classes are denoted by $\langle p\rangle$. On $\mathbb{R}^{2}$, the notion of primary and semi-primary coincide since $\mathbb{R}^{2}$ is contractible. Thus, assume from now on that $(M, \omega)$ is a surface with genus $g \geq 1$.

We define the semi-primary Floer chain complex via

$$
\left.\tilde{C}_{k}:=\tilde{C}(x, \varphi):=\bigoplus_{\substack{\langle p\rangle \in \tilde{\mathcal{H}}_{s} \\ \mu(\langle p\rangle)=k}} \mathbb{Z}\langle p\rangle, \quad \tilde{\partial}\langle p\rangle:=\sum_{\substack{\langle q\rangle \in \tilde{\mathcal{H}}_{s} \\ \mu(\langle q\rangle)=\mu(\langle p\rangle)-1}} m(\langle p\rangle,\langle q\rangle)\langle q\rangle\right)
$$

and extend the boundary operator linearly to $\tilde{\partial}: \tilde{C}_{*} \rightarrow \tilde{C}_{*-1}$. 
Theorem 57. It holds $\tilde{\partial} \circ \tilde{\partial}=0$ and $\tilde{H}_{*}(x, \varphi):=\operatorname{ker} \tilde{\partial}_{*} / \operatorname{Im} \tilde{\partial}_{*+1}$ is called semi-primary Floer homology.

Proof. Since the set of contractible semiprimary points is a subset of the set of primary points most of the proofs for primary Floer homology carry over. However, we have to check the cutting procedure: since we are restricting the boundary operator to the subset $\mathcal{H}_{s} \subset \mathcal{H}_{p r}$ of the primary points we have to make sure that the cutting points are also in $\mathcal{H}_{s}$.

More precisely, we have to proof an analogon of Proposition 29 and Theorem 30 for contractible semiprimary points. This can be deduced from the already existing primary classification Proposition 29 and Figure 5 as follows:

Let $p, r \in \mathcal{H}_{s}$ with $\mu(p, r)=2$. Since $p$ and $r$ are contractible and semiprimary, they satisfy $] p, x\left[_{s} \cap\right] p, x\left[_{u}=\emptyset=\right] r, x\left[{ }_{s} \cap\right] r, x\left[{ }_{u}\right.$ and both 'loops' $[p, x]_{s} \cup[p, x]_{u}$ and $[r, x]_{s} \cup[r, x]_{u}$ span a 2-gon. This yields us the same pictures and positioning for $p$ and $r$ as in Figure 5, but this time on the manifold and not on the universal cover. Then we notice that we have 2gons with vertices $p$ and $r$ of relative Maslov index \pm 2 in the middle column of Figure 5 and 2-gons with vertices $x$ and $r$ resp. $p$ of relative index \pm 3 in the right and left column of Figure 5. We observe that, in the left and right column, the cutting procedure takes place within this 2-gon of relative Maslov index \pm 3 . Thus it does not matter if we are on the universal cover or on the manifold itself - the cutting works in the very same way as for primary points and provides us with two contractible semiprimary cutting points $q_{s}$ and $q_{u}$.

Now let us consider the middle column. Remember, the 2 -gon with vertices $r$ and $p$ lies on the manifold. Consider the small 'overlapping nose'. $q_{s}$ and $q_{u}$ are not primary whether or not the 'overlapping nose' wraps around some genus like in Figure 14. And neither are they contractible semiprimary.

Thus we reproved the classification in Figure 5 for contractible semiprimary points which implies an analogon of the cutting procedure Theorem 30 (and of Lemma 19). Altogether, we deduce $\tilde{\partial} \circ \tilde{\partial}=0$ and the existence of semiprimary Floer homology.

The difference of $\mathcal{H}_{p r}$ and $\mathcal{H}_{s}$ is only noticeable on manifolds with genus and their different properties come apparent in the following example. A brief look at Figure 14 tells us that, on the one hand, a semi-primary point is lost as generator since the move turns $q$ from (semi-)primary to primary and that, on the other hand, the new arising semi-primary point $s$ is not contractible. This observation leads to

Example 58. Consider the example of Figure 14 and assume for simplicity, that only the branches containing $p$ intersect (which is entirely possible if 
$M$ is not closed). Then the semi-primary Floer homology is given by

$$
\tilde{H}_{-1}(x, \varphi) \simeq \mathbb{Z} \quad \text { and } \quad \tilde{H}_{*}(x, \varphi)=0 \text { otherwise. }
$$

Let $\sigma(n)=-1$ for $n \in \mathbb{Z}^{>0}$ and $\sigma(n)=1$ for $n \in \mathbb{Z}^{<0}$. Then we obtain

$$
\tilde{H}_{\sigma(n)}\left(x, \varphi^{n}\right) \simeq \mathbb{Z}^{n} \text { and } \tilde{H}_{*}\left(x, \varphi^{n}\right)=0 \text { otherwise. }
$$

Proof. In Figure 14, $p$ is (semi-)primary and contractible with $\mu(p)=-1$. $q$ is primary (and contractible) with $\mu(q)=-2$, but not semi-primary. $s$ is semi-primary and not contractible. $r$ is secondary and not contractible. Thus we obtain $\tilde{C}_{-1}(x, \varphi)=\mathbb{Z}\langle p\rangle$ and $\tilde{C}_{*}(x, \varphi)=0$ otherwise. The boundary operator is given by $\tilde{\partial}\langle p\rangle=0$ and thus $\tilde{H}_{*}(x, \varphi)=\tilde{C}_{*}(x, \varphi)$.

Now we consider iterates of the symplectomorphism. For $n \in \mathbb{Z}^{>0}$, we obtain the complex $\tilde{C}_{-1}\left(x, \varphi^{n}\right)=\operatorname{Span}_{\mathbb{Z}}\left\{\left\langle p^{0}\right\rangle, \ldots,\left\langle p^{n-1}\right\rangle\right\}$ and $\tilde{C}_{*}\left(x, \varphi^{n}\right)=$ 0 otherwise. The boundary operator is given by $\tilde{\partial}\left\langle p^{l}\right\rangle=0$ for $0 \leq l \leq n-1$ and thus $\tilde{H}_{*}\left(x, \varphi^{n}\right)=\tilde{C}_{*}\left(x, \varphi^{n}\right) \simeq \mathbb{Z}^{n}$. For $n \in \mathbb{Z}^{<0}, W^{s}$ and $W^{u}$ are exchanged which leads to the change of the Maslov index.

The computation of primary Floer homology for $\varphi$ and $\varphi^{n}$ for the example in Figure 14 was partially done before Theorem 50 and in the proof of Proposition 56 and we recall

$$
H_{-1}(x, \varphi)=C_{-1}(x, \varphi) \simeq \mathbb{Z} \text { and } H_{-2}(x, \varphi)=C_{-2}(x, \varphi) \simeq \mathbb{Z} .
$$

For higher iterates with $n \in \mathbb{Z}^{>0}$, we found $C_{-1}\left(x, \varphi^{n}\right)=\operatorname{Span}_{\mathbb{Z}}\left\{\left\langle p^{0}\right\rangle, \ldots\right.$, $\left.\left\langle p^{n-1}\right\rangle\right\}$ and $C_{-2}\left(x, \varphi^{n}\right)=\operatorname{Span}_{\mathbb{Z}}\left\{\left\langle q^{0}\right\rangle \ldots\left\langle q^{n-1}\right\rangle\right\}$ and $H_{-1}\left(x, \varphi^{n}\right)=H_{-1}$ $(x, \varphi) \simeq \mathbb{Z}$ and $H_{-2}\left(x, \varphi^{n}\right)=H_{-2}(x, \varphi) \simeq \mathbb{Z}$. For negative $n$, we have $H_{2}\left(x, \varphi^{n}\right) \simeq \mathbb{Z}$ and $H_{1}\left(x, \varphi^{n}\right) \simeq \mathbb{Z}$.

As long as all primary points are also semi-primary, $H_{*}(x, \varphi)$ and $\tilde{H}_{*}(x, \varphi)$ coincide. The difference becomes apparent as soon as a move circles around some genus and turns a semi-primary point primary. For primary Floer homology, this kind of move is in fact secondary. The arising of $r$ and $s$ turns $q$ from semi-primary to primary which is not noticed by primary Floer homology. Semi-primary Floer homology is sensitive to this move since it means the loss of a generator.

The distinction between homoclinic points $p$ with contractible or noncontractible loop $[x, p]_{u} \cup[x, p]_{s}$ arise naturally in systems on the torus or cylinder resp. annulus. Hockett \& Holmes $[\mathbf{H H}]$ study the existence and impact of such (semi-primary) homoclinic points on the annulus. If $[x, p]_{u} \cup[x, p]_{s}$ is contractible they call $p$ non-rotary. If $[x, p]_{u} \cup[x, p]_{s}$ winds $k$ times around the hole of the annulus, they call $p k$-rotary. Noncontractible, semi-primary points therefore fit as 1-rotary orbits in their framework.

6.4. Chaotic Floer homology. The difference of primary Floer homology and semi-primary Floer homology is due to their different generator sets. In this subsection, we define a version of homoclinic Floer homology which 
is based on primary points as generators, but whose boundary operator is different from the one in primary Floer homology.

We want to include some of the nearby chaos in the definition of homoclinic Floer homology. Before we start, recall some classical results about the existence of periodic points near a homoclinic tangle. Birkhoff [Bi] proved in 1935 that there is an intricate amount of (mostly high)periodic points near a homoclinic one which was formalized by Smale's horseshoe. For periodic points, there is Conley's conjecture which claims the existence of infinitely many periodic points on certain symplectic manifolds. By now, it has been established for certain manifolds, cf. Ginzburg [Gi], Hingston $[\mathbf{H i}]$.

Now we will define a homoclinic Floer homology which takes also periodic points of the underlying symplectomorphism into account. Assume $\varphi \in \operatorname{Diff}_{\omega}(M)$ and $x \in \operatorname{Fix}(x)$ hyperbolic. Depending on the iteration number $n \in \mathbb{Z}$, we assign new signs to primary points $p, q \in \mathcal{H}\left(\varphi^{n}, x\right)$ via

$$
\nu_{n}(p, q):= \begin{cases}m(p, q) & \text { if } \emptyset \neq \mathcal{M}(p, q) \ni u, \operatorname{Fix}\left(\varphi^{n}\right) \cap \operatorname{Im}(u)=\emptyset \\ 0 & \text { otherwise. }\end{cases}
$$

Set $\nu_{n}(\langle p\rangle,\langle q\rangle):=\sum_{l \in \mathbb{Z}} \nu_{n}\left(p, q^{l}\right)$ and define the chain complexes as $\mathscr{C}_{*}^{(n)}:=$ $C_{*}\left(x, \varphi^{n} ; \mathbb{Z}\right)$. The boundary operators are

$$
\left.\mathscr{D}^{(n)}: \mathscr{C}_{*}^{(n)} \rightarrow \mathscr{C}_{*-1}^{(n)}, \quad \mathscr{D}^{(n)}(\langle p\rangle):=\sum_{\substack{\langle q\rangle \in \tilde{\mathcal{H}}_{p r}\left(\varphi^{n}\right) \\ \nu_{n}(\langle p\rangle,\langle q\rangle)=1}} \nu_{n}(\langle p\rangle,\langle q\rangle)\langle q\rangle\right)
$$

on a generator and are extended to $\mathscr{D}^{(n)}$ by linearity.

Theorem 59. It holds $\mathscr{D}^{(n)} \circ \mathscr{D}^{(n)}=0$ and $\hat{H}_{*}\left(x, \varphi^{n}\right):=\hat{H}_{*}\left(x, \varphi^{n} ; \mathbb{Z}\right):=$ $\frac{\operatorname{ker} \mathscr{D}_{*}^{(n)}}{\operatorname{Im} \mathscr{D}_{*+1}^{(n)}}$ is called chaotic Floer homology.

Proof. Compared to primary Floer homology, chaotic Floer homology also uses primary points as generators, but employs a modified boundary operator which counts 'less' digons than the one of primary Floer homology. Thus the gluing procedure and also the finiteness of the sum in the definition of the boundary operator carry directly over from primary Floer homology.

And also the cutting procedure is still valid: Let $n \in \mathbb{N}$ and consider the primary points of $\varphi^{n}$. For $p, r \in \mathcal{H}_{p r}\left(\varphi^{n}\right)$ with $\mu(p, r)=2$, Theorem 30 yields the two cutting points $q_{s}, q_{u} \in \mathcal{H}_{p r}\left(\varphi^{n}\right)$ since counting or not counting a di-gon with the new $\operatorname{sign} \nu_{n}(\cdot, \cdot)$ is independent from the existence of the cutting points.

Thus we only have to prove an analogon of Lemma 19 for the new signs $\nu_{n}(\cdot, \cdot)$. Consider the possible cutting situations in Figure 16. If there are no fixed points of $\varphi^{n}$ in the ranges of the involved di-gons as in Figure 16 (a), the signs $\nu_{n}(\cdot, \cdot)$ coincide with the signs $m(\cdot, \cdot)$ and Lemma 19 holds true. 
(a)

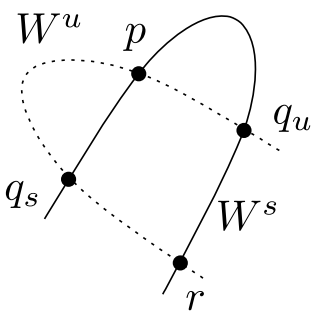

(b)

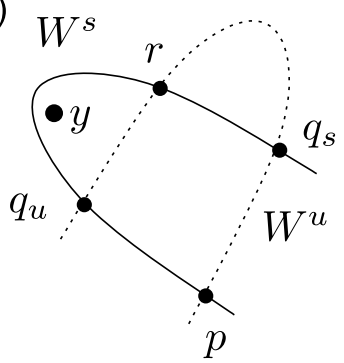

Figure 16. Signs in chaotic Floer homology.

Now assume that there are fixed point(s) in the range(s). For instance, as in Figure $16(\mathrm{~b})$, let $y \in \operatorname{Fix}\left(\varphi^{n}\right)$ lie in the range of all di-gons in $\mathcal{M}\left(p, q_{s}\right)$, but not in the range of the di-gons in $\mathcal{M}\left(q_{s}, r\right)$. We compute

$$
\begin{array}{r}
\nu_{n}\left(p, q_{s}\right) \cdot \nu_{n}\left(q_{s}, r\right)=0 \cdot \nu_{n}\left(q_{s}, r\right)=0, \\
-\nu_{n}\left(p, q_{u}\right) \cdot \nu_{n}\left(q_{u}, r\right)=-\nu_{n}\left(p, q_{u}\right) \cdot 0=0 .
\end{array}
$$

Other placements of (possibly several) fixed points yield similar calculations. Thus we proved an analogon of Lemma 19 which yields the claim.

Remark 60. (1) Chaotic primary Floer homology is invariant under conjugation.

(2) The additional condition on the signs renders an invariance discussion for arbitrary high $n$ futile since the fixed point condition prevents moves. Invariance makes only sense for fixed $n$ and then one would have to require the existence of continuations of all involved periodic points.

The main importance of chaotic Floer homology lies in its change under iteration. Therefore let us consider the dynamics of $n \mapsto \hat{H}_{*}\left(x, \varphi^{n}\right)$ in an explicit example.

Recall the notation $\varphi^{n}(p)=: p^{n}$ with $p=p^{0}$. Let $\varphi \in \operatorname{Diff}_{\omega}\left(\mathbb{R}^{2}\right)$ have the homoclinic tangle sketched in Figure 17 (i) and assume the following additional data: Let $\operatorname{Fix}(\varphi)=\{x, y\}$ and set $\operatorname{Per}_{k}(\varphi)$ to be the set of periodic points whose smallest period is $k$. Assume $\operatorname{Per}_{2}(\varphi)=\left\{z^{0}, z^{1}\right\}$ and $\operatorname{Per}_{3}(\varphi)=$ $\emptyset$ and that only the branches containing $p$ intersect. The homoclinic tangles of $\varphi^{2}$ and $\varphi^{3}$ are drawn in Figure 17 (ii) and (iii) where we have splitted $x$ into two copies. Assume the positions of $x, y, z^{0}$ and $z^{1}$ as in Figure 17 . Now we compute the chaotic Floer homology for $n \in\{1,2,3\}$. 
(i)

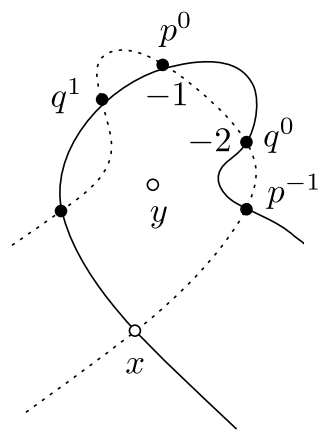

(ii)

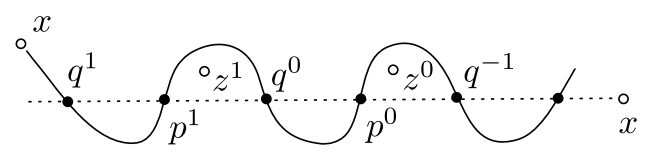

(iii)

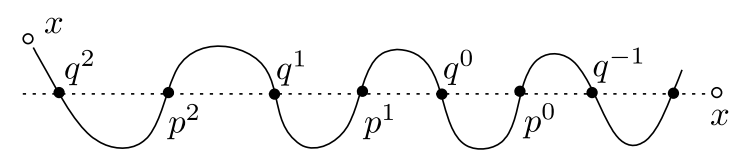

Figure 17. Chaos near the homoclinic tangle.

Example 61. Under the above assumption, we obtain

$$
\begin{array}{ll}
\text { For } n=1: & \hat{H}_{-1}(x, \varphi) \simeq \mathbb{Z} \text { and } \quad \hat{H}_{-2}(x, \varphi) \simeq \mathbb{Z}, \\
\text { For } n=2: & \hat{H}_{-1}\left(x, \varphi^{2}\right)=0 \quad \text { and } \quad \hat{H}_{-2}\left(x, \varphi^{2}\right)=0, \\
\text { For } n=3: & \hat{H}_{-1}\left(x, \varphi^{3}\right) \simeq \mathbb{Z} \quad \text { and } \quad \hat{H}_{-2}\left(x, \varphi^{3}\right) \simeq \mathbb{Z} .
\end{array}
$$

Proof. For $n=1$ we have $\mathscr{C}_{-1}^{(1)}=\mathbb{Z}\langle p\rangle$ and $\mathscr{C}_{-2}^{(1)}=\mathbb{Z}\langle q\rangle$. The boundary operator is $\mathscr{D}^{(1)}\langle p\rangle=-\langle q\rangle+\langle q\rangle=0$ and $\mathscr{D}^{(1)}\langle q\rangle=0$. Thus $\hat{H}_{*}(x, \varphi) \simeq \mathscr{C}_{*}^{(1)}$.

For $n=2$ we obtain $\mathscr{C}_{-1}^{(2)}=\mathbb{Z}\left\langle p^{0}\right\rangle \oplus \mathbb{Z}\left\langle p^{1}\right\rangle$ and $\mathscr{C}_{-2}^{(2)}=\mathbb{Z}\left\langle q^{0}\right\rangle \oplus \mathbb{Z}\left\langle q^{1}\right\rangle$. The boundary operator is given by $\mathcal{D}^{(2)}\left\langle p^{0}\right\rangle=\left\langle q^{0}\right\rangle$ and $\mathcal{D}^{(2)}\left\langle p^{1}\right\rangle=\left\langle q^{1}\right\rangle$. This yields $\hat{H}_{-1}\left(x, \varphi^{2}\right)=0$ and $\hat{H}_{-2}\left(x, \varphi^{2}\right)=0$.

For $n=3$, there is

$$
\begin{aligned}
& \mathscr{C}_{-1}^{(3)}=\mathbb{Z}\left\langle p^{0}\right\rangle \oplus \mathbb{Z}\left\langle p^{1}\right\rangle \oplus \mathbb{Z}\left\langle p^{2}\right\rangle, \quad \mathscr{D}^{(3)}\left\langle p^{0}\right\rangle=\left\langle q^{0}\right\rangle-\left\langle q^{2}\right\rangle, \quad \mathscr{D}^{(3)}\left\langle q^{0}\right\rangle=0, \\
& \mathscr{C}_{-2}^{(3)}=\mathbb{Z}\left\langle q^{0}\right\rangle \oplus \mathbb{Z}\left\langle q^{1}\right\rangle \oplus \mathbb{Z}\left\langle q^{2}\right\rangle, \quad \mathscr{D}^{(3)}\left\langle p^{1}\right\rangle=\left\langle q^{1}\right\rangle-\left\langle q^{0}\right\rangle, \quad \mathscr{D}^{(3)}\left\langle q^{1}\right\rangle=0, \\
& \mathscr{D}^{(3)}\left\langle p^{2}\right\rangle=\left\langle q^{2}\right\rangle-\left\langle q^{1}\right\rangle, \quad \mathscr{D}^{(3)}\left\langle q^{2}\right\rangle=0
\end{aligned}
$$

and we compute $\hat{H}_{-1}\left(x, \varphi^{3}\right) \simeq \mathbb{Z} \simeq \hat{H}_{-2}\left(x, \varphi^{3}\right)$.

Recall that we calculated the primary Floer homology for the iterates of $\varphi$ in Proposition 56 and obtained $H_{-1}\left(x, \varphi^{n}\right) \simeq \mathbb{Z}$ and $H_{-2}\left(x, \varphi^{n}\right) \simeq \mathbb{Z}$ for all $n \in \mathbb{Z}^{>0}$ which was oblivious to the iteration.

This simple example demonstrates the properties of chaotic Floer homology very well. For the higher iterates we know that $z \in \operatorname{Fix}(\varphi)$ implies $z \in \operatorname{Fix}\left(\varphi^{n}\right)$ and $z \in \operatorname{Fix}\left(\varphi^{l}\right) \cap \operatorname{Fix}\left(\varphi^{k}\right)$ implies $z \in \operatorname{Fix}\left(\varphi^{k \cdot l}\right)$. However, apart from those 'old ones', new fixed points might or will arise according to our discussion above. 
The dynamical behaviour of $n \mapsto \hat{H}_{*}\left(x, \varphi^{n}\right)$ leads to a symplectic zeta function

$$
\zeta_{x, \varphi}(z):=\exp \left(\sum_{n=1}^{\infty} \frac{\chi\left(\hat{H}_{*}\left(x, \varphi^{n}\right)\right)}{n} z^{n}\right)
$$

where $\chi\left(\hat{H}_{*}\left(x, \varphi^{n}\right)\right)$ denotes the Euler characteristic of $\hat{H}_{*}\left(x, \varphi^{n}\right)$. Zeta function have been studies a lot in number theory, algebraic geometry and dynamics. For an overview see for instance Fel'shtyn $[\mathbf{F e 1}, \mathbf{F e} 2]$.

Question 62. (a) Is there $\varphi$ such that $\zeta(x, \varphi)$ is rational? If yes, which $\varphi$ ?

(b) Is there a relation to the classical (symplectic) zeta function?

(c) Are there applications to Nielsen theory and Reidemeister torsion whose relation to dynamical zeta functions is described in Fel'shtyn [Fe1]?

\section{References}

[AS] A. Abbondandolo and M. Schwarz, On the Floer homology of cotangent bundles, Comm. Pure Appl. Math. 59(2) (2006), 254-316.

[Ba] A. Banyaga, Sur la structure du groupe des difféomorphismes qui préserve une forme symplectique, Comm. Math. Helv. 53 (1978), 174-227.

[Bi] G.D. Birkhoff, Nouvelles recherches sur les systèmes dynamiques, Mem. Pont. Acad. Sci. Nov. Lyncaei 1935, 53, 85-216.

[Br] G. Bredon, Topology and geometry, Springer 1993.

[Che] Y. Chekanov, Differential algebra of Legendrian links, Invent. Math. 150 (2002), 441-483.

[dS] S. de Silva, Products in the symplectic Floer homology of Lagrangian intersections, Thesis, Merton College, Oxford, 1998.

[Fe1] A. Fel'shtyn, Dynamical Zeta functions and Floer homology, Contemporary Mathematics 385 (2005), 187-203.

[Fe2] A. Fel'shtyn, Dynamical Zeta functions, Nielsen theory and Reidemeister torsion, Memoirs AMS (2001), 147(699).

[Fl1] A. Floer, A relative Morse index for the symplectic action, Comm. Pure Appl. Math. 41 (1988), 393-407.

[Fl2] A. Floer, The unregularized gradient flow of the symplectic action, Comm. Pure Appl. Math. 41 (1988), 775-813.

[Fl3] A. Floer, Morse theory for Lagrangian intersections, J. Diff. Geom. 28 (1988), 513-547.

[FO3] K. Fukaya, Y.-G. Oh, H. Ohta and K. Ono, Lagrangian intersection Floer theory: anomaly and obstruction, Parts I and II, American Mathematical Society, Providence, RI, 2009.

[GauRS] R. Gautschi, J. Robbin and D. Salamon, Heegard splittings and Morse-Smale flows, Int. J. Math. Math. Sci. (56)(2003), 3539-3572.

[Gi] V.L. Ginzburg, The Conley Conjecture, Ann. Math. (2) 172(2) (2010), 1127-1180. 
[GiG] V. Ginzburg and B. Gürel, Action and index spectra and periodic orbits in Hamiltonian dynamics, Geom. Topol. 13 (2009), 2745-2805.

[GH] J. Guckenheimer and P. Holmes, Nonlinear oscillations, dynamical systems, and bifurcations of vector fields, Springer, 3rd edn. 1990.

[Hi] N. Hingston, Subharmonic solutions of Hamiltonian equations on tori, Annal. Math. 170(2) (2009), 529-560.

[HH] K. Hockett and P. Holmes, Josephson's junction, annulus maps, Birkhoff attractors, horseshoes and rotation sets, Ergod. Th. Dyn. Syst. 6 (1986), 205-239.

[Ho1] S. Hohloch, Floer homology for homoclinic tangles, Dissertation, University of Leipzig, 2008.

[Ho2] S. Hohloch, Transport, flux and growth of homoclinic Floer homology, Discrete Contin. Dyn. Syst. Series A 32(1) (2012), 3587-3620.

[MMP] R. MacKay, J. Meiss and I. Percival, Transport in Hamiltonian systems, Physica D (1984), 55-81.

[Ma1] J. Mather, A criterion for the non-existence of invariant circles, Publ. IHES (1986), 153-204.

[McS1] D. McDuff and D. Salamon, Introduction to Symplectic Topology, Clarendon Press, Oxford 1998.

[Me] V.K. Melnikov, On the stability of the center for time periodic perturbations, Trans. Moscow Math. Soc. 12 (1963), 1-57.

[Mi] J. Milnor, Topology from the differentiable viewpoint, Princeton University Press, Princeton, NJ. 1997.

[Ol] F. Oliveira, On the $C^{\infty}$ genericity of homoclinic orbits, Nonlinearity 13 (2000), 653-662.

[Pa] J. Palis, On Morse-Smale dynamical systems, Topology 8 (1969), 385-405.

[Poi1] H. Poincaré, Sur le problème des trois corps et les équations de la dynamique, Acta Math., 13 (1890), 1-271.

[Poi2] H. Poincaré, Les méthodes nouvelles de la mechanique céleste, Gauthier-Villars, Paris 1899.

[Pol1] L. Polterovich, On transport in dynamical systems (russian), Usp. Mat. Nauk 43(1) (259) 1988.

[Pol2] L. Polterovich, The geometry of the group of symplectic diffeomorphism, Birkhäuser 2001.

[Pol3] L. Polterovich, Growth of maps, distortion of groups and symplectic geometry, Inv. Math. 150 (2002), 655-686.

[Pol4] L. Polterovich, Floer homology, dynamics and groups, in P. Biran et al. (eds) Morse theoretic methods in nonlinear analysis and in symplectic topology 417438, Springer 2006.

[Ro] J. Robbin, Heegard splittings and Floer homology, Preprint September 2000.

[RK1] V. Rom-Kedar, Homoclinic tangles - classification and applications, Nonlinearity 7 (1994), 441-473.

[RK2] V. Rom-Kedar, Secondary homoclinic bifurcation theorems, Chaos 5(2) (1995), $385-401$. 
[Sa] D. Salamon, Lectures on Floer homology, in: Symplectic geometry and topology (Park City, UT, 1997), 143-229, IAS/Park City Math. Ser., 7, Amer. Math. Soc., Providence, RI, 1999.

[Sm1] S. Smale, A structurally stable differentialbe homeomorphism with an infinite number of periodic points, in: Proceedings of the International Symposium on nonlinear Oscillatons, Izdat. Akad. Nauk Ukrain SSR, Vol. 2, Kiev, 1963, 365-366.

[Sm2] S. Smale, Diffeomorphisms with many periodic points, Differential and combinatorial topology, Princeton University Press, Princeton, NJ, 1965, 63-80.

[Ta] F. Takens, Homoclinic points in conservative systems, Invent. Math. 18 (1972), 267-292.

[Xia1] Z. Xia, Homoclinic points in symplectic and volume-preserving diffeomorphisms, Commun. Math. Phys. 177 (1996), 435-449.

[Xia2] Z. Xia, Homoclinic points and intersections of Lagrangian submanifolds, Discr. Cont. Dyn. Syst. 6(1) (2000), 243-253.

[Xia3] Z. Xia, Homoclinic points for area preserving surface diffeomorphisms, Preprint 2006 arXiv:Math $\backslash 0606291 \mathrm{v} 1$.

Ecole Polytechnique FÉdérale de Lausanne

SB MATHGEOM CAG

1015 LAUSANNE, SwitzerLAND

E-mail address: sonja.hohloch@epfl.ch

http://sma.epfl.ch/ hohloch/

Received 04/06/2012, accepted 12/12/2012

The author wishes to thank G. Noetzel, M. Schwarz, Z. Xia and E. Zehnder for helpful discussions, explanations and references. 
